Pacific

Journal of

Mathematics

\title{
ON TWO NOTIONS OF SEMISTABILITY
}

\author{
MARIO MAICAN
}




\title{
ON TWO NOTIONS OF SEMISTABILITY
}

\author{
MARIO MAICAN
}

\begin{abstract}
We show that certain semistable sheaves on the projective plane with linear Hilbert polynomial are cokernels of semistable morphisms of decomposable bundles. We exhibit certain locally closed subvarieties or open dense subsets of moduli spaces of semistable sheaves as quotients modulo nonreductive groups. These subvarieties are defined by cohomological conditions. We find isomorphisms between such subvarieties given by sending a sheaf to its dual.
\end{abstract}

\section{Introduction}

The notion of a (Gieseker) semistable sheaf is well-established in the literature and allows one to construct moduli spaces of sheaves with fixed Hilbert polynomial on a projective variety. The construction, carried out in [Simpson 1994a; 1994b], relies on the existence theorems from geometric invariant theory, more precisely, it is shown that the moduli space occurs as the quotient of a certain set of semistable points of a quotient scheme modulo a reductive algebraic group.

To get a semistable quotient from a semistable sheaf $\mathscr{F}_{F}$ we need to express $\mathscr{F}$ as a quotient $m \mathscr{O}(-d) \rightarrow \mathscr{F} \rightarrow 0$ with large $m$ and $d$. In general this procedure is quite abstract and of little use for the purposes of describing concretely the geometry of the moduli space.

Another approach for studying moduli spaces uses monads. Let $\mathrm{M}_{\mathbb{P}^{2}}\left(r, c_{1}, c_{2}\right)$ be the moduli space of semistable (in the sense of Mumford and Takemoto) torsionfree sheaves on $\mathbb{P}^{2}$ of rank $r$ and Chern classes $c_{1}, c_{2}$. Assume that there exist locally free sheaves $\mathscr{E}_{1}, \mathscr{E}_{2}, \mathscr{E}_{3}$ on $\mathbb{P}^{2}$ such that each $\mathscr{F}$ giving a point in $\mathrm{M}_{\mathbb{P}^{2}}\left(r, c_{1}, c_{2}\right)$ is the cohomology of a monad

$$
0 \rightarrow \mathscr{E}_{1} \rightarrow \mathscr{E}_{2} \rightarrow \mathscr{E}_{3} \rightarrow 0 .
$$

The space $W$ of monads is acted upon in an obvious manner by the algebraic group $G=$ Aut $\mathscr{E}_{1} \times$ Aut $\mathscr{E}_{2} \times$ Aut $\mathscr{E}_{3}$. Two fundamental questions now arise: Is there a semistability notion for $W$ such that a monad is semistable precisely if its

MSC2000: 14F05, 14L24, 00A05, 14D20, 14D22.

Keywords: moduli spaces, nonreductive groups, sheaves on the projective plane, semistable sheaves, sheaves of dimension one. 
cohomology is semistable? And is $\mathrm{M}_{\mathbb{P}^{2}}\left(r, c_{1}, c_{2}\right)$ a good quotient of the set $W^{\text {ss }}$ of semistable monads modulo $G$ ?

The description of $\mathbf{M}_{\mathbb{P}^{2}}\left(2, c_{1}, c_{2}\right)$ as a good quotient was done in [Barth 1977] for $c_{1}$ even and in [Hulek 1979] for $c_{1}$ odd. In [Chang 1983] it was shown that a generic stable bundle on $\mathbb{P}^{3}$ of rank 2, Chern classes $c_{1}=0, c_{2}=4$ and $\alpha$-invariant 1 is the cohomology of a self-dual monad. Drézet [1987] described as quotients those $\mathrm{M}_{\mathbb{P}^{2}}\left(r, c_{1}, c_{2}\right)$ for which $\Delta=\delta$. He takes $\mathscr{E}_{3}=0$ and $\mathscr{E}_{1}, \mathscr{E}_{2}$ direct sums of certain exceptional bundles. In all these instances the group $G$ was reductive. Quotients by nonreductive $G$ were considered in [Drézet 1991], which studies $\mathbf{M}_{\mathbb{P}^{2}}\left(r, c_{1}, c_{2}\right)$ of "faible hauteur". Again $\mathscr{E}_{3}=0$, so Drézet is able to express each semistable bundle as the cokernel of a semistable morphism.

A notion of semistability for complexes of morphisms of sheaves modulo nonreductive groups was proposed in [Drézet 1991; 1998; Drézet and Trautmann 2003]. We briefly explain the case of morphisms of sheaves. (In this paper we will not need the notion of a semistable complex of length 3 or more.) Drézet and Trautmann consider sheaves $\mathscr{E}_{1}$ and $\mathscr{E}_{2}$ on $\mathbb{P}^{n}$ which are direct sums of simple sheaves, e.g., direct sums of line bundles. Thus Aut $\mathscr{E}_{1} \times$ Aut $\mathscr{E}_{2}$ is nonreductive if $\mathscr{E}_{1}$ or $\mathscr{E}_{2}$ has more than one kind of simple sheaf in its decomposition. This group acts on the vector space $W=\mathscr{H o m}\left(\mathscr{E}_{1}, \mathscr{E}_{2}\right)$ and the set of semistable points $W^{\text {ss }}$ is defined by means of polarizations which will be not detailed here. We refer to Section 3 for the precise definition. In [Drézet and Trautmann 2003] as well as in [Drézet 2000] it was shown that this notion of semistability quite often leads to a theory similar to the geometric invariant theory.

Freiermuth and Trautmann [2004] studied the moduli space of semistable (in the sense of Gieseker) sheaves $\mathscr{F}_{F}$ on $\mathbb{P}^{3}$ with Euler characteristic 1 and with support curves of multiplicity 3 . They showed that each $\mathscr{F}_{F}$ has a resolution

$$
0 \rightarrow 2 \mathrm{O}(-3) \stackrel{\psi}{\rightarrow} \mathrm{O}(-1) \oplus 3 \mathcal{O}(-2) \stackrel{\varphi}{\rightarrow} \mathrm{O}(-1) \oplus \mathcal{O} \rightarrow \mathscr{F} \rightarrow 0
$$

with $\varphi$ semistable in the sense of [Drézet and Trautmann 2003]. Moreover, the moduli space is a geometric quotient of the parameter space of $(\psi, \varphi)$ modulo the action of the group of automorphisms.

In this paper we are interested in semistable sheaves on $\mathbb{P}^{2}$ with linear Hilbert polynomial. Let $\mathrm{M}_{\mathbb{P} 2}(r, \chi)$ denote the moduli space of such sheaves $\mathscr{F}$ with fixed multiplicity $r$ and Euler characteristic $\chi$. Motivated by [Freiermuth and Trautmann 2004] we will seek to express $\mathscr{F}$ as a cokernel

$$
\stackrel{\mathscr{E}}{\mathscr{E}_{1}} \stackrel{\varphi}{\rightarrow} \mathscr{\mathscr { E }}_{2} \rightarrow \mathscr{F} \rightarrow 0
$$

with $\mathscr{E}_{1}$ and $\mathscr{E}_{2}$ direct sums of line bundles and $\varphi$ semistable in the sense of Drézet and Trautmann. We carry this out in Sections 4-6 for sheaves satisfying certain 
cohomological conditions. The picture we provide is far from complete because we do not have a full list of resolutions for all $\mathscr{F}$ giving a point in $\mathbf{M}_{\mathbb{P}^{2}}(r, \chi)$ even in the case $r=4$ (the cases $r=1,2$ are trivial while the case $r=3$ is completely understood).

Our cohomological conditions define locally closed subvarieties in $\mathbf{M}_{\mathbb{P}^{2}}(r, \chi)$ and in Section 7 we address the question whether these subvarieties are good or geometric quotients of the sets of semistable morphisms $\varphi$ modulo the canonical action of the group of automorphisms. We find that when $r, \chi$ are mutually prime, in other words when $\mathrm{M}_{\mathbb{P}^{2}}(r, \chi)$ is a fine moduli space, we always have geometric quotients. If the moduli space is not fine the problem is more complicated and we can answer it only in some cases.

In Section 8 we compute the codimensions of all locally closed subsets of $\mathbf{M}_{\mathbb{P}^{2}}(r, \chi)$ under investigation.

In Section 9 we prove a general duality result. The dual of a sheaf $\mathscr{F}$ giving a point in $\mathrm{M}_{\mathbb{P}^{2}}(r, \chi)$ is $\mathscr{F}^{D}=\mathscr{E} x t^{1}\left(\mathscr{F}_{F}, \Omega^{2}\right)(1)$. Applying the map $\mathscr{F}_{F} \rightarrow \mathscr{F}^{D}$ to a locally closed subset $X$ in $\mathbf{M}_{\mathbb{P}^{2}}(r, \chi)$ we get a locally closed subset in $\mathbf{M}_{\mathbb{P}^{2}}(r, r-\chi)$ denoted $X^{D}$. At Theorem 9.6 we show that under certain conditions $X$ and $X^{D}$ are isomorphic. In particular, this is true for all sets $X$ under investigation in this paper. Our theorem is inspired from the result present in [Freiermuth 2000], that $\mathrm{M}_{\mathbb{P}^{2}}(r, \chi)$ and $\mathrm{M}_{\mathbb{P}^{2}}(r, r-\chi)$ are birational if $\operatorname{gcd}(r, \chi)=1$. We show that this is also true if $(r, \chi)=(6,4),(8,6)$, or $(9,6)$.

We summarize our results in the tables that follow. The first column of each table, after the header, contains the cohomological conditions defining a locally closed subset $X \subset \mathrm{M}_{\mathbb{P}^{2}}(r, \chi)$. The second shows the codimension of $X$; a zero means an open dense subset. Each sheaf $\mathscr{F}$ giving a point in $X$ has a resolution of the kind featured in the header. We have more information about these resolutions: the morphisms $\varphi$ of which $\mathscr{F}$ is the cokernel form a subset $W_{o}$ inside the set $W^{\text {ss }}(G, \Lambda)$ of morphisms which are semistable with respect to a polarization $\Lambda$ and to the canonical action of the group $G$ of automorphisms (see Section 3 for the terminology). The third column of our tables contains information about $\Lambda$ and the forth column says whether $X$ is a quotient of $W_{o}$ by $G$. When we write "good" it is understood that the quotient is not geometric; a question mark means we could not prove that a quotient exists. The subset $W_{o} \subset W^{\mathrm{ss}}(G, \Lambda)$ is given by the following conditions: for all the blocks different than the last block in the table we require that $\varphi$ be injective and that its scalar entries (regarding it as a matrix) are zero. For the last block we refer to Claims 6.9 and 6.10.

\begin{tabular}{|l|c|l|c|}
\hline \multicolumn{2}{|c|}{$\mathrm{M}_{\mathbb{P}^{2}}(n+1, n), n \geq 1$} & \multicolumn{2}{c|}{$0 \rightarrow \mathcal{O}(-2) \oplus(n-1) \mathcal{O}(-1) \rightarrow n \mathscr{O} \rightarrow \mathscr{F} \rightarrow 0$} \\
\hline$h^{0}(\mathscr{F}(-1))=0$ & 0 & $0<\lambda_{1}<\frac{1}{n}$ & geometric \\
\hline
\end{tabular}




\begin{tabular}{|c|c|c|c|}
\hline \multicolumn{2}{|c|}{$\mathrm{M}_{\mathbb{P}^{2}}(n+2, n), n=3,4,5}$, & \multicolumn{2}{|c|}{$0 \rightarrow 20(-2) \oplus(n-2) \mathcal{O}(-1) \rightarrow n \mathcal{O} \rightarrow \mathscr{F} \rightarrow 0$} \\
\hline $\begin{array}{l}h^{0}(\mathscr{F}(-1))=0 \\
h^{1}(\mathscr{F})=0 \\
h^{1}\left(\mathscr{F} \otimes \Omega^{1}(1)\right)=0\end{array}$ & 0 & $\frac{1}{2 n}<\lambda_{1}<\frac{1}{n}$ & $\begin{array}{l}n=4,6: \text { good } \\
n=3,5: \text { geometric }\end{array}$ \\
\hline \multicolumn{4}{|c|}{$0 \rightarrow 2 \mathcal{O}(-2) \oplus(n-1) \mathcal{O}(-1) \rightarrow \mathcal{O}(-1) \oplus n \mathcal{O} \rightarrow \mathscr{F} \rightarrow 0$} \\
\hline $\begin{array}{l}h^{0}(\mathscr{F}(-1))=0 \\
h^{1}(\mathscr{F})=0 \\
h^{1}\left(\mathscr{F} \otimes \Omega^{1}(1)\right)=1\end{array}$ & $n-1$ & $\begin{array}{l}\left(\lambda_{1}, \mu_{1}\right) \text { in the interior of the } \\
\text { triangle with vertices }(0,0) \text {, } \\
(\alpha, \alpha),(\beta, 2 \beta), \text { where } \\
\alpha=1 /(n+1), \beta=1 /\left(n^{2}-n+2\right)\end{array}$ & $\begin{array}{l}n=3,5: \text { geometric } \\
n=4,6: ?\end{array}$ \\
\hline
\end{tabular}

\begin{tabular}{|c|c|c|c|}
\hline \multicolumn{2}{|l|}{$\mathbf{M}_{\mathbb{P}^{2}}(4,2)$} & \multicolumn{2}{|c|}{$0 \rightarrow 2 \mathrm{O}(-2) \rightarrow 2 \mathrm{O} \rightarrow \mathscr{F} \rightarrow 0$} \\
\hline $\begin{array}{l}h^{0}(\mathscr{F}(-1))=0 \\
h^{1}(\mathscr{F})=0 \\
h^{1}\left(\mathscr{F} \otimes \Omega^{1}(1)\right)=0\end{array}$ & 0 & $\lambda_{1}=\frac{1}{2}$ & good \\
\hline \multicolumn{4}{|c|}{$0 \rightarrow 2 \mathcal{O}(-2) \oplus \mathcal{O}(-1) \rightarrow \mathscr{O}(-1) \oplus 2 \mathcal{O} \rightarrow \mathscr{F} \rightarrow 0$} \\
\hline $\begin{array}{l}h^{0}(\mathscr{F}(-1))=0 \\
h^{1}(\mathscr{F})=0 \\
h^{1}\left(\mathscr{F} \otimes \Omega^{1}(1)\right)=1\end{array}$ & 1 & $\begin{array}{l}\left(\lambda_{1}, \mu_{1}\right) \text { in the interior of the } \\
\text { quadrilateral with vertices }(0,0) \text {, } \\
\left(\frac{1}{3}, \frac{1}{3}\right),\left(\frac{1}{2}, 1\right),(0,1)\end{array}$ & $?$ \\
\hline
\end{tabular}

\begin{tabular}{|c|c|c|c|}
\hline \multicolumn{2}{|c|}{$\mathbf{M}_{\mathbb{P}^{2}}(n+3, n), n=4,5,6$} & \multicolumn{2}{|c|}{$0 \rightarrow 30(-2) \oplus(n-3) \mathcal{O}(-1) \rightarrow n \mathscr{O} \rightarrow \mathscr{F} \rightarrow 0$} \\
\hline $\begin{array}{l}h^{0}(\mathscr{F}(-1))=0 \\
h^{1}(\mathscr{F})=0 \\
h^{1}\left(\mathscr{F} \otimes \Omega^{1}(1)\right)=0\end{array}$ & 0 & $\frac{2}{3 n}<\lambda_{1}<\frac{1}{n}$ & $\begin{array}{l}n=4,5: \text { geometric } \\
n=6: ?\end{array}$ \\
\hline \multicolumn{4}{|c|}{$0 \rightarrow 3 \mathcal{O}(-2) \oplus(n-2) \mathcal{O}(-1) \rightarrow \mathcal{O}(-1) \oplus n \mathscr{O} \rightarrow \mathscr{F} \rightarrow 0$} \\
\hline $\begin{array}{l}h^{0}(\mathscr{F}(-1))=0 \\
h^{1}(\mathscr{F})=0 \\
h^{1}\left(\mathscr{F} \otimes \Omega^{1}(1)\right)=1\end{array}$ & $n-2$ & see Claim 4.7 & $\begin{array}{l}n=4,5: \text { geometric } \\
n=6: ?\end{array}$ \\
\hline
\end{tabular}

\begin{tabular}{|c|c|c|c|}
\hline $\mathbf{M}_{\mathbb{P}^{2}}(7,4)$ & & \multicolumn{2}{|c|}{$0 \rightarrow 30(-2) \oplus 3 \mathcal{O}(-1) \rightarrow 20(-1) \oplus 4 \mathbb{O} \rightarrow \mathscr{F} \rightarrow 0$} \\
\hline $\begin{array}{l}h^{0}(\mathscr{F}(-1))=0 \\
h^{1}(\mathscr{F})=0 \\
h^{1}\left(\mathscr{F} \otimes \Omega^{1}(1)\right)=2\end{array}$ & 6 & $\begin{array}{l}\left(\lambda_{1}, \mu_{1}\right) \text { in the interior of the } \\
\text { quadrilateral with vertices }(0,0) \text {, } \\
\left(\frac{1}{3}, \frac{1}{2}\right),\left(\frac{17}{24}, 1\right),(1,1)\end{array}$ & geometric \\
\hline
\end{tabular}




\begin{tabular}{|c|c|c|c|}
\hline \multicolumn{2}{|l|}{$\mathbf{M}_{\mathbb{P} 2}(6,3)$} & \multicolumn{2}{|c|}{$0 \rightarrow 30(-2) \rightarrow 30 \rightarrow \mathscr{F} \rightarrow 0$} \\
\hline $\begin{array}{l}h^{0}(\mathscr{F}(-1))=0 \\
h^{1}(\mathscr{F})=0 \\
h^{1}\left(\mathscr{F} \otimes \Omega^{1}(1)\right)=0\end{array}$ & 0 & $\lambda_{1}=\frac{1}{3}$ & good \\
\hline \multicolumn{4}{|c|}{$0 \rightarrow 3 \mathfrak{O}(-2) \oplus \mathscr{O}(-1) \rightarrow \mathcal{O}(-1) \oplus 3 \mathfrak{O} \rightarrow \mathscr{F} \rightarrow 0$} \\
\hline $\begin{array}{l}h^{0}(\mathscr{F}(-1))=0 \\
h^{1}(\mathscr{F})=0 \\
h^{1}\left(\mathscr{F} \otimes \Omega^{1}(1)\right)=1\end{array}$ & 1 & $\begin{array}{l}\left(\lambda_{1}, \mu_{1}\right) \text { in the interior of the } \\
\text { segment with endpoints }\left(\frac{1}{4}, \frac{1}{4}\right) \text {, } \\
\left(\frac{1}{5}, \frac{2}{5}\right)\end{array}$ & $?$ \\
\hline \multicolumn{4}{|c|}{$0 \rightarrow 30(-2) \oplus 20(-1) \rightarrow 20(-1) \oplus 3 \mathcal{O} \rightarrow \mathscr{F} \rightarrow 0$} \\
\hline $\begin{array}{l}h^{0}(\mathscr{F}(-1))=0 \\
h^{1}(\mathscr{F})=0 \\
h^{1}\left(\mathscr{F} \otimes \Omega^{1}(1)\right)=2\end{array}$ & 4 & $\begin{array}{l}\left(\lambda_{1}, \mu_{1}\right) \text { in the interior of the } \\
\text { triangle with vertices }(0,0) \text {, } \\
\left(\frac{1}{5}, \frac{1}{5}\right),\left(\frac{1}{3}, \frac{1}{2}\right)\end{array}$ & $?$ \\
\hline \multicolumn{4}{|c|}{$0 \rightarrow \mathscr{O}(-3) \oplus 3 \mathcal{O}(-1) \rightarrow 4 \mathbb{O} \rightarrow \mathscr{F} \rightarrow 0$} \\
\hline $\begin{array}{l}h^{0}(\mathscr{F}(-1))=0 \\
h^{1}(\mathscr{F})=1\end{array}$ & 4 & $0<\lambda_{1}<\frac{1}{4}$ & geometric \\
\hline \multicolumn{4}{|c|}{$0 \rightarrow 40(-2) \rightarrow 30(-1) \oplus \mathscr{O}(1) \rightarrow \mathscr{F} \rightarrow 0$} \\
\hline $\begin{array}{l}h^{0}(\mathscr{F}(-1))=1 \\
h^{1}(\mathscr{F})=0\end{array}$ & 4 & $0<\mu_{2}<\frac{1}{4}$ & geometric \\
\hline
\end{tabular}

\begin{tabular}{|l|c|c|c|}
\hline \multicolumn{3}{|c|}{$0 \rightarrow \mathcal{O}(-3) \oplus \mathscr{O}(-1) \rightarrow 2 \mathcal{O} \rightarrow \mathscr{F} \rightarrow 0$} \\
\hline $\mathrm{M}_{\mathbb{P}^{2}}(4,1)$ & $0<\lambda_{1}<\frac{1}{2}$ & geometric \\
$h^{0}(\mathscr{F}(-1))=0$ & 2 & $\mathscr{F})=1$ & 0 \\
\hline
\end{tabular}

\begin{tabular}{|l|c|c|c|}
\hline \multicolumn{3}{|c|}{$0 \rightarrow \mathcal{O}(-3) \oplus 2 \mathcal{O}(-1) \rightarrow 3 \mathcal{O} \rightarrow \mathscr{F} \rightarrow 0$} \\
\hline $\mathrm{M}_{\mathbb{P} 2}(5,2)$ & $0<\lambda_{1}<\frac{1}{3}$ & geometric \\
$h^{1}(\mathscr{F})=1$ & 3 & $0.1)=0$ & \\
\hline
\end{tabular}

\begin{tabular}{|c|c|c|c|}
\hline \multicolumn{2}{|c|}{$\mathrm{M}_{\mathbb{P} 2}(n, 2), 4 \leq n \leq 15$} & \multicolumn{2}{|c|}{$\begin{aligned} 0 \rightarrow \mathcal{O}(-2) \rightarrow(n-2) \mathcal{O}(-2) \oplus 3 \mathcal{O}(-1) \\
\quad \rightarrow(n-3) \mathbb{O}(-1) \oplus 3 \mathcal{O} \rightarrow \mathscr{F} \rightarrow 0\end{aligned}$} \\
\hline $\begin{array}{l}h^{0}(\mathscr{F}(-1))=1 \\
h^{1}(\mathscr{F})=0\end{array}$ & $n-2$ & $\begin{array}{l}\left(\lambda_{1}, \mu_{1}\right) \text { in the interior of the } \\
\text { triangle with vertices }(0,0) \text {, } \\
\left(\frac{1}{n}, \frac{1}{n}\right),\left(\frac{1}{n-2}, \frac{1}{n-3}\right)\end{array}$ & $\begin{array}{l}3 \nmid n \text { : geometric } \\
3 \mid n: ?\end{array}$ \\
\hline
\end{tabular}


By virtue of our duality results Theorem 9.6 and Proposition 9.10, for each block in the table there is a "dual block" obtained by replacing $\mathrm{M}_{\mathbb{P}^{2}}(r, \chi)$ with $\mathrm{M}_{\mathbb{P}^{2}}(r, r-\chi)$, F्F with $\mathscr{F}^{D}$ and $\varphi$ with $\mathscr{H}$ om $\left(\varphi, \Omega^{2}\right)(1)$. We did not feel the need to include another "dual" table, instead we only spell out in Corollary 9.11 the cases of open dense subsets.

As a general remark, the kind of arguments from this paper become very hard to carry out in the case of large multiplicity. This is so because, when the multiplicity becomes large, other than semistability conditions on $\varphi$ enter into play. Thus, for large multiplicity, Drézet and Trautmann's notion of semistability is no longer satisfactory.

\section{Semistable sheaves and their moduli}

From now on $k$ will be an algebraically closed field of characteristic zero. All schemes over $k$ will be assumed to be algebraic, meaning that they can be covered with finitely many spectra of finitely generated $k$-algebras. A separated algebraic scheme will also be called an algebraic variety. A variety will be the maximal spectrum of a reduced algebraic variety. Our main reference for this section is [Huybrechts and Lehn 1997].

Let $X$ be a smooth projective variety of dimension $n$ with ample line bundle $\mathrm{O}_{X}(1)$. For a coherent sheaf $\mathscr{F}$ on $X$ we denote by $\chi(\mathscr{F})$ its Euler characteristic given by

$$
\chi(\mathscr{F})=\sum_{i \geq 0}(-1)^{i} \operatorname{dim}_{k} H^{i}(X, \mathscr{F}) .
$$

The Euler characteristic of the twisted sheaf $\mathscr{F}(m)=\mathscr{F} \otimes \mathcal{O}_{X}(m)$ is a polynomial expression in $m$. Thus, we can define the Hilbert polynomial $P_{\mathscr{F}}(m)$ of $\mathscr{F}$ by the formula

$$
P_{\mathscr{F}}(m)=\chi(\mathscr{F}(m)) .
$$

It is known that the degree of $P_{\mathscr{F}}(m)$ equals the dimension of the topological support $\operatorname{supp}(\mathscr{F})$ of $\mathscr{F}$. We write

$$
P_{\mathscr{F}}(m)=\sum_{i=0}^{d} \alpha_{i}(\mathscr{F}) \frac{m^{i}}{i !} .
$$

The coefficients $\alpha_{i}(\mathscr{F})$ are integers, see [Huybrechts and Lehn 1997]. The dominant coefficient $\alpha_{d}(\mathscr{F})$ is called the multiplicity of $\mathscr{F}$ and is positive because, by the Theorem B of Serre, for $m$ large enough we have $P_{\mathscr{F}}(m)=\operatorname{dim}_{k} H^{0}(X, \mathscr{F}(m))>0$. It is known that $\alpha_{d}(\mathscr{F})$ equals the degree of the $\operatorname{scheme} \operatorname{Supp}(\mathscr{F})$ which has $\operatorname{supp}(\mathscr{F})$ as underlying topological space and $\mathscr{O}_{X} / \mathscr{A} n n(\mathscr{F})$ as structure sheaf. We define the reduced Hilbert polynomial of $\mathscr{F}$ as 


$$
p_{\mathscr{F}}=\frac{P_{\mathscr{F}}}{\alpha_{d}(\mathscr{F})} .
$$

Definition 2.1. Let $\mathscr{F}$ be a coherent sheaf on $X$. Assume that $\operatorname{Supp}(\mathscr{F})$ is pure dimensional of dimension $d$. We say that $\mathscr{F}$ is semistable (stable) if the following two conditions are satisfied:

(i) $\mathscr{F}$ does not have nonzero subsheaves $\mathscr{F}^{\prime}$ with $\operatorname{Supp}\left(\mathscr{F}^{\prime}\right)$ having dimension smaller than $d$;

(ii) for any proper subsheaf $\mathscr{F}^{\prime} \subset \mathscr{F}$ we have

$$
p_{\mathscr{F}^{\prime}} \leq(<) p_{\mathscr{F}}
$$

meaning that for $m$ sufficiently large the following inequality holds:

$$
p_{\mathscr{F}^{\prime}}(m) \leq(<) p_{\mathscr{F}}(m) .
$$

Remark 2.2. We will be interested in semistable sheaves on $\mathbb{P}^{2}$ with linear Hilbert polynomial $P_{\mathscr{F}}(m)=r m+\chi$. Such sheaves are supported on projective curves $C$ and the conditions from the above definition take the form:

(i) $\mathscr{F}$ does not have zero-dimensional torsion;

(ii) for any proper subsheaf $\mathscr{F}^{\prime} \subset \mathscr{F}$ we have

$$
\frac{\alpha_{0}\left(\mathscr{F}^{\prime}\right)}{\alpha_{1}\left(\mathscr{F}^{\prime}\right)}(\leq)<\frac{\alpha_{0}(\mathscr{F})}{\alpha_{1}(\mathscr{F})} .
$$

We point out that $\mathscr{F}$ is a torsion $\mathrm{O}_{\mathbb{P}^{2}}$-module because at every point $x$ there is a

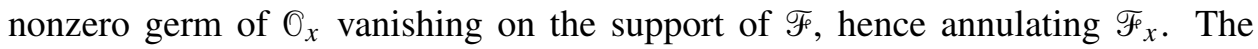
zero-dimensional torsion of a sheaf is its largest subsheaf supported on finitely many points.

The positive integer $r$ is the so-called multiplicity of $\mathscr{F}$ while $\chi$ is its Euler characteristic. The restriction of $\mathscr{F}_{F}$ to a generic line in $\mathbb{P}^{2}$ is a sheaf of length $r$ supported at finitely many points; $r$ is also equal to the degree of $C$. Here are more facts about such sheaves (compare [Freiermuth 2000, Theorem 3.1]):

Proposition 2.3. Let $\mathscr{F}$ be a semistable sheaf on $\mathbb{P}^{n}$ with Hilbert polynomial $P_{\mathscr{F}}(m)=r m+\chi, 0 \leq \chi<r$. Let $C$ be its support. Then:

(i) F is Cohen-Macaulay;

(ii) $\mathscr{F}$ is locally free on the smooth part of $C$;

(iii) $C$ has no zero-dimensional components and no embedded points;

(iv) if $\operatorname{gcd}(r, \chi)=1$ then $\mathscr{F}$ is stable;

(v) if $h^{0}(\mathscr{F}(-1))=0$ then $h^{1}(\mathscr{F}(i))=0$ for $i \geq r-\chi-1$. 
As a generic plane curve is smooth, we see that a generic $\mathscr{F}$ from Proposition 2.3 is a line bundle supported on a smooth curve of degree $r$. Its degree can be computed with the Riemann-Roch formula: $\operatorname{deg}(\mathscr{F})=g(C)-1+\chi=\frac{1}{2} r(r-3)+\chi$. Line bundles supported on smooth curves are clearly stable because their quotient sheaves are supported on finitely many points, hence their proper subsheaves have the same multiplicity but strictly smaller Euler characteristic. Other, well-known, examples of stable sheaves with one-dimensional support are the structure sheaves ${ }^{O_{C}}$, where $C$ is any curve in $\mathbb{P}^{2}$ given as the zero-set of a polynomial of degree $r$. We can see this using, for instance, Lemma 6.7: any ideal sheaf $\mathscr{I} \subset \mathscr{O}_{C}$ has Hilbert polynomial

$$
P_{\mathscr{O}_{C}}(t)-P_{\mathscr{O}_{C^{\prime}}}(t)-a=r t-\frac{r(r-3)}{2}-r^{\prime} t+\frac{r^{\prime}\left(r^{\prime}-3\right)}{2}-a,
$$

where $a \geq 0$ and $r^{\prime}<r$ are integers. Thus

$$
\frac{\alpha_{0}(\Im)}{\alpha_{1}(\Im)}=\frac{-r-r^{\prime}+3}{2}-\frac{a}{r-r^{\prime}}<\frac{-r+3}{2}=\frac{\alpha_{0}\left(\mathscr{O}_{C}\right)}{\alpha_{1}\left(\mathscr{O}_{C}\right)} .
$$

Definition 2.4. Let $\mathscr{F}$ be a semistable sheaf on X. A Jordan-Hölder filtration of $\mathscr{F}$ is a filtration by subsheaves

$$
0=\mathscr{F}_{0} \varsubsetneqq \mathscr{F}_{1} \varsubsetneqq \ldots \varsubsetneqq \mathscr{F}_{r}=\mathscr{F}
$$

such that all quotients $\mathscr{F}_{i} / \mathscr{F}_{i-1}$ are stable with reduced Hilbert polynomial $p_{\mathscr{F}}$. Two semistable sheaves $\mathscr{F}$ and $\mathscr{G}$ on $X$ are said to be stable equivalent if they posess Jordan-Hölder filtrations with isomorphic quotients. By this we mean the following: there is a bijection between the set of quotients of the filtration of $\mathscr{F}$ and the set of quotients of the filtration of $\mathscr{G}$ such that the quotients corresponding via this bijection are isomorphic.

In the case of stable sheaves, stable equivalence means isomorphism. Any semistable sheaf $\mathscr{F}$ has at least one Jordan-Hölder filtration. $\mathscr{F}$ may have more than one filtration, but the direct sum $\oplus \mathscr{F}_{i} / \mathscr{F}_{i-1}$ does not depend on the filtration.

The moduli space of semistable sheaves on $X$ parametrizes stable equivalence classes with fixed Hilbert polynomial. It is a coarse moduli space for a certain moduli problem that can be defined by means of the following functor: Fix a numerical polynomial $P(m)$, i.e., a polynomial with rational coefficients taking integer values on the integers. For any scheme $S$ over $k$ we define $\mu_{X}(P)(S)$ as the set of equivalence classes [F्F] of $S$-flat coherent sheaves $\mathscr{F}$ on $S \times X$ whose restriction $\mathscr{F}_{S}$ to any fiber $\pi^{-1}(s)$ is a semistable sheaf with Hilbert polynomial $P$. Here $\pi: S \times X \rightarrow S$ is the projection onto the first factor. Two sheaves $\mathscr{F}$ and $\mathscr{G}$ on $S \times X$ are said to be equivalent if there is a line bundle $\mathscr{L}$ on $S$ such that $\mathscr{F}$ is isomorphic to $\mathscr{G} \otimes \pi^{*} \mathscr{L}$. Given a morphism $f: T \rightarrow S$ of schemes over $k$ we have 
a map

$$
\mu_{X}(P)(f): \mathcal{M}_{X}(P)(S) \rightarrow \mu_{X}(P)(T)
$$

given by the pull-back

$$
M_{X}(P)(f)([\mathscr{F}])=\left[(f \times 1)^{*} \mathscr{F}\right] .
$$

We have thus defined a countervariant functor $\mu_{X}(P)$ from the category of schemes over $k$ to the category of sets.

Definition 2.5. A scheme $M$ over $k$ is called a coarse moduli space of semistable sheaves on $X$ with Hilbert polynomial $P$ if there is a natural transformation of functors

$$
M_{X}(P)(-) \stackrel{\tau}{\rightarrow} \operatorname{Mor}(-, M)
$$

satisfying the following properties:

(i) the map

$$
\tau(\operatorname{Spec} k): M_{X}(P)(\operatorname{Spec} k) \rightarrow \operatorname{Mor}(\operatorname{Spec} k, \mathrm{M})
$$

is a bijection. In other words the set of closed points of $M$ is in a one-toone correspondence with the set of stable equivalence classes of semistable sheaves on $X$ with Hilbert polynomial $P$;

(ii) given a scheme $\mathrm{N}$ and a natural transformation

$$
M_{X}(P)(-) \stackrel{\tau^{\prime}}{\rightarrow} \operatorname{Mor}(-, N)
$$

there is a unique morphism $f: \mathrm{M} \rightarrow \mathrm{N}$ such that $f \circ \tau(S)=\tau^{\prime}(S)$ for all $S$.

Theorem 2.6. Let $X$ be a smooth projective variety with ample line bundle $O_{X}(1)$. Let $P$ be a numerical polynomial. Then:

(i) there exists a coarse moduli space $\mathrm{M}_{X}(P)$ of semistable sheaves on $X$ with Hilbert polynomial $P$;

(ii) $\mathrm{M}_{X}(P)$ is a projective scheme;

(iii) there is an open subscheme $\mathrm{M}_{X}^{s}(P)$ of $\mathrm{M}_{X}(P)$ whose closed points parametrize the isomorphism classes of stable sheaves with Hilbert polynomial $P$.

The theorem in its full generality was proved in [Simpson 1994a]. We recall the way $\mathrm{M}_{X}(P)$ is constructed: For a suitably large integer $m$ let $V$ be a vector space of dimension $P(m)$. We consider the quotient scheme

$$
Q=\operatorname{Quot}\left(X, V \otimes \mathcal{O}_{X}(-m), P\right)
$$

of coherent sheaves $\mathscr{F}$ on $X$ with Hilbert polynomial $P$ which occur as quotients $V \otimes \mathcal{O}_{X}(-m) \rightarrow \mathscr{F}$. The reductive group $\operatorname{SL}(V)$ acts on $Q$ by its action on the first component of $V \otimes \mathrm{O}_{X}(-m)$. Inside $Q$ there is the open and $\operatorname{SL}(V)$-invariant 
subset $R$ of semistable points. Semistability here is meant in the sense of geometric invariant theory, which also guarantees the existence of a categorical quotient (see Definition 7.1) of $R$ by $\operatorname{SL}(V)$. This quotient is the moduli space $\mathrm{M}_{X}(P)$.

We now turn to the question under which circumstances the moduli space of stable sheaves $\mathrm{M}_{X}^{s}(P)$ is fine. Fine moduli spaces represent certain functors, so we define the countervariant functor $M_{X}^{s}(P)$ from the category of schemes over $k$ to the category of sets in the same way as $\mu_{X}(P)$ was defined, with the difference that we now require each restriction $\mathscr{F}_{s}$ to be stable.

Definition 2.7. We say that $\mathrm{M}_{X}^{s}(P)$ is a fine moduli space of stable sheaves on $X$ with Hilbert polynomial $P$ if the natural transformation

$$
M_{X}^{s}(P)(-) \stackrel{\tau^{s}}{\rightarrow} \operatorname{Mor}\left(-, M_{X}^{s}(P)\right)
$$

induced by $\tau$ is an isomorphism of functors. If this is true, let $U$ be the sheaf on $\mathrm{M}_{X}^{s} \times X$ whose class $[u] \in M_{X}^{s}(P)\left(\mathrm{M}_{X}^{s}(P)\right)$ corresponds under $\tau$ to the identity map of $\mathrm{M}_{X}^{s}(P)$. We say that $U$ is a universal family on $\mathrm{M}_{X}^{s}(P)$.

Remark 2.8. The inverse of $\tau^{s}(S)$ for a scheme $S$ is given by $\tau^{s}(S)^{-1}(f)=\left[f^{*} u\right]$. In fact, $\mathbf{M}_{X}^{s}(P)$ is a fine moduli space if and only if there exists a coherent sheaf $\mathcal{U}$ on $\mathrm{M}_{X}^{s}(P) \times X$ such that:

(i) $U$ is flat over $\mathrm{M}_{X}^{s}(P)$;

(ii) for any point $[\mathscr{F}] \in \mathrm{M}_{X}^{s}(P)$ the restriction of $\mathcal{U}$ to the fiber $[\mathscr{F}] \times X$ is isomorphic to $\mathscr{F}$

(iii) $U$ has the following universality property: for any scheme $S$ over $k$ and any $S$ flat coherent family $\mathscr{F}$ of semistable sheaves on $S \times X$ with Hilbert polynomial $P$, there exists a unique morphism $f: S \rightarrow \mathrm{M}_{X}^{S}(P)$ such that $\mathscr{F} \simeq f^{*} U \otimes \pi^{*} \mathscr{L}$ for some line bundle $\mathscr{L}$ on $S$.

Theorem 2.9. Consider the numerical polynomial

$$
P(m)=\sum_{i=0}^{d} \alpha_{i}\left(\begin{array}{c}
m+i-1 \\
i
\end{array}\right) .
$$

Assume that $\operatorname{gcd}\left(\alpha_{0}, \ldots, \alpha_{d}\right)=1$. Then $\mathrm{M}_{\mathbb{P} n}^{s}(P)$ is a fine moduli space for any $n \geq d$.

We refer to [Huybrechts and Lehn 1997] for the proof of this theorem. In this paper we will focus on moduli spaces of sheaves on $\mathbb{P}^{2}$ with linear Hilbert polynomial $P_{\mathscr{F}}(m)=r m+\chi$. To bring us closer to the notations from [Le Potier 1993], where such moduli spaces were systematically studied, we also write $\mathbf{M}_{\mathbb{P} n}(r, \chi)$ instead of $\mathbf{M}_{\mathbb{P} n}(r m+\chi)$, respectively $\mathbf{M}_{\mathbb{P} n}^{s}(r, \chi)$ instead of $\mathbf{M}_{\mathbb{p} n}^{s}(r m+\chi)$. Combining Theorem 2.9 with Proposition 2.3(iv) we obtain: 
Proposition 2.10. Assume that $\operatorname{gcd}(r, \chi)=1$. Then $\mathrm{M}_{\mathbb{P} n}(r, \chi)=\mathrm{M}_{\mathbb{p n}}^{s}(r, \chi)$ is a fine moduli space.

From theorem 3.19(2) in [Le Potier 1993] we learn that $\mathbf{M}_{\mathbb{P} 2}^{s}(r, \chi)$ is not a fine moduli space in the case when $r$ and $\chi$ are not mutually prime. We quote the precise statement (which is stronger):

Theorem 2.11. If $r$ and $\chi$ are not mutually prime, then for any open subset $U \subset \mathrm{M}_{\mathbb{P}^{2}}(r, \chi)$ there is no universal sheaf on $U \times \mathbb{P}^{2}$.

As the spaces $\mathbf{M}_{\mathbb{P}^{2}}(r, \chi)$ and $\mathbf{M}_{\mathbb{P}^{2}}(r, r+\chi)$ are isomorphic, we will assume henceforth that $0<\chi \leq r$. Theorem 3.1 and Proposition 2.3 from [Le Potier 1993] yield the following:

Theorem 2.12. For any integers $r \geq 1$ and $\chi$ the moduli space $\mathrm{M}_{\mathbb{P}^{2}}(r, \chi)$ is irreducible, of dimension $r^{2}+1$ and smooth on the open dense set represented by stable sheaves.

We finish this section with an easy observation about subsets of moduli spaces:

Remark 2.13. Let $\mathscr{E}$ be a locally free sheaf on $X$. For any integers $i, j \geq 0$ the subset of $\mathrm{M}_{X}(P)$ of stable equivalence classes of sheaves $\mathscr{F}$ with $h^{i}(X, \mathscr{F} \otimes \mathscr{E}) \geq j$ is a closed algebraic subset.

Proof. Using the notations preceeding Definition 2.7, we consider the universal quotient $V \otimes \mathscr{O}_{X}(-m) \rightarrow \widetilde{\mathscr{F}}$ on $Q \times X$. The sheaf $\widetilde{\mathscr{F}} \otimes \mathscr{E}$ is flat over $Q$ so, according to the semicontinuity theorem, the set $Y$ of equivalence classes of quotients $V \otimes$ $\mathrm{O}_{X}(-m) \rightarrow \mathscr{F}_{F}$ with $h^{i}\left(X, \mathscr{F}_{\mathbb{F}} \otimes \mathscr{E}\right) \geq j$ is a closed algebraic subset in $Q$. Notice that $Y$ is $\mathrm{SL}(V)$-invariant so, by virtue of property (iv) (Definition 7.2), its image under the good quotient map $R \rightarrow \mathrm{M}_{X}(P)$ is closed. This image is precisely the subset from the remark.

If $\mathrm{M}_{X}(P)$ is a fine moduli space, then the remark follows directly from the semicontinuity theorem applied to $\mathscr{\bigotimes} \otimes \mathscr{E}$.

\section{Semistable morphisms of sheaves}

Given coherent sheaves $\mathscr{E}$ and $\mathscr{F}$ on $\mathbb{P}^{n}$ the affine space $W=\operatorname{Hom}(\mathscr{E}, \mathscr{F})$ is acted upon by the algebraic group $G=$ Aut $\mathscr{E} \times$ Aut $\mathscr{F} / k^{*}$. Here $k^{*}$ is embedded as the group of homotheties $\left\{\left(t \cdot 1_{\mathscr{E}}, t \cdot 1_{\mathscr{F}}\right), t \in k^{*}\right\}$. The action is given by $(g, h) . w=$ $h \circ w \circ g^{-1}$. If $G$ is reductive then geometric invariant theory distinguishes an open subset $W^{\text {ss }} \subset W$ of so-called semistable morphisms and constructs a categorical quotient $W^{\text {ss }} / / G$. Our difficulty is that in general $G$ is not reductive. A notion of semistability in the context of nonreductive groups has been studied in [Drézet and Trautmann 2003] and its usefulness has been made clear in [Drézet 2000]. 
This section introduces Drézet and Trautmann's notion of semistability and is mainly a reproduction of notations from [Drézet and Trautmann 2003]. Fix sheaves

$$
\mathscr{E}=\oplus_{1 \leq i \leq r} M_{i} \otimes \mathscr{E}_{i}, \quad \mathscr{F}_{F}=\oplus_{1 \leq l \leq s} N_{l} \otimes \mathscr{F}_{l}
$$

where $M_{i}, N_{l}$ are vector spaces over $k$ of dimensions $m_{i}, n_{l}$ while $\mathscr{E}_{i}, \mathscr{F}_{l}$ are simple sheaves on $\mathbb{P}^{n}$, meaning that their only endomorphisms are homotheties. For our purposes $\mathscr{E}_{i}$ and $\mathscr{F}_{l}$ will be line bundles. We assume that $\operatorname{Hom}\left(\mathscr{E}_{i}, \mathscr{E}_{j}\right)=0$ when $i>j$ and $\operatorname{Hom}\left(\mathscr{F}_{l}, \mathscr{F}_{m}\right)=0$ when $l>m$. We denote

$$
\begin{aligned}
H_{l i} & =\operatorname{Hom}\left(\mathscr{E}_{i}, \mathscr{F}_{l}\right), \\
A_{j i} & =\operatorname{Hom}\left(\mathscr{E}_{i}, \mathscr{E}_{j}\right), \\
B_{m l} & =\operatorname{Hom}\left(\mathscr{F}_{l}, \mathscr{F}_{m}\right) .
\end{aligned}
$$

The group $G$ consists of pairs of matrices $(g, h)$,

$$
g=\left[\begin{array}{ccccc}
g_{1} & 0 & \cdots & \cdots & 0 \\
u_{21} & g_{2} & \cdots & \cdots & 0 \\
\vdots & & \ddots & & \vdots \\
\vdots & & \ddots & \vdots \\
u_{r 1} & & \cdots & u_{r, r-1} & g_{r}
\end{array}\right], \quad h=\left[\begin{array}{ccccc}
h_{1} & 0 & \cdots & \cdots & 0 \\
v_{21} & h_{2} & \cdots & \cdots & 0 \\
\vdots & & \ddots & & \vdots \\
\vdots & & \ddots & \vdots \\
v_{s 1} & & \cdots & v_{s, s-1} & h_{s}
\end{array}\right]
$$

with

$g_{i} \in \mathrm{GL}\left(M_{i}\right), h_{l} \in \mathrm{GL}\left(N_{l}\right), u_{j i} \in \operatorname{Hom}\left(M_{i}, M_{j} \otimes A_{j i}\right), \quad v_{m l} \in \operatorname{Hom}\left(N_{l}, N_{m} \otimes B_{m l}\right)$.

The conditions $u_{i j}=0$ and $v_{m l}=0$ define a reductive subgroup $G_{r e d}$ inside $G$.

For fixed positive integers $\lambda_{i}, \mu_{l}$ we consider the character $\chi$ of $G$ given by

$$
\chi(g, h)=\prod_{1 \leq i \leq r} \operatorname{det}\left(g_{i}\right)^{-\lambda_{i}} \cdot \prod_{1 \leq l \leq s} \operatorname{det}\left(h_{l}\right)^{\mu_{l}} .
$$

Since $\chi$ must be trivial on the subgroup of homotheties $k^{*}$, we impose the relation

$$
\sum_{1 \leq i \leq r} m_{i} \lambda_{i}=\sum_{1 \leq l \leq s} n_{l} \mu_{l}
$$

and we denote by $d$ this sum. We will call a polarization the tuple

$$
\Lambda=\left(\lambda_{1}, \ldots, \lambda_{r}, \mu_{1}, \ldots, \mu_{s}\right) .
$$

Definition 3.1. Let $\Lambda$ be a fixed polarization. A point $w \in W$ is called:

(i) semistable with respect to $G_{\text {red }}$ and $\Lambda$ if there are $n \geq 1$ and a polynomial $f \in k[W]$ satisfying $f(g . x)=\chi^{n}(g) f(x)$ for all $g \in G_{\text {red }}, x \in W$, such that $f(w) \neq 0$; 
(ii) stable with respect to $G_{r e d}$ and $\Lambda$ if $\operatorname{Stab}_{G_{r e d}}(w)$ is zero-dimensional and there is $f$ as above but with the additional property that the action of $G_{r e d}$ on $W_{f}=\{x \in W, f(x) \neq 0\}$ is closed;

(iii) properly semistable if it is semistable but not stable.

The question is now how to define semistability with respect to $G$. The key is the following observation from geometric invariant theory: let $T$ be a maximal torus inside a reductive algebraic group which acts on a projective variety. Then a point on the variety is semistable if and only if all points in its orbit are semistable with respect to $T$. In our context the subgroup of diagonal matrices is a maximal torus inside both $G_{r e d}$ and $G$. This justifies the following:

Definition 3.2. A point $w \in W$ is called (semi)stable with respect to $G$ and $\Lambda$ if $g . w$ is (semi)stable with respect to $G_{\text {red }}$ and $\Lambda$ for all $g \in G$. We denote by $W^{\text {ss }}(G, \Lambda), W^{s}(G, \Lambda)$ the corresponding sets.

To describe the sets of semistable points in concrete situations we will use a very special case of King's criterion of semistability as formulated in [Drézet and Trautmann 2003]. Write

$$
\mathscr{E}=\oplus_{j} \mathscr{\mathscr { E }}_{j}^{\prime}, \quad \mathscr{F}=\oplus_{m} \mathscr{F}_{m}^{\prime}
$$

where $\mathscr{E}_{j}^{\prime}, \mathscr{F}_{m}^{\prime}$ are line bundles. We represent $w$ by a matrix $\left(w_{m j}\right)$ with $w_{m j} \in$ $\operatorname{Hom}\left(\mathscr{E}_{j}^{\prime}, \mathscr{F}_{m}^{\prime}\right)$. We put

$$
\lambda_{j}^{\prime}=\lambda_{i} \quad \text { if } \mathscr{E}_{j}^{\prime} \simeq \mathscr{E}_{i}, \quad \mu_{m}^{\prime}=\mu_{l} \quad \text { if } \mathscr{F}_{m}^{\prime} \simeq \mathscr{F}_{l}
$$

Proposition 3.3. A morphism $w \in W$ is (semi)stable with respect to $G$ and $\Lambda$ if and only if for all $g \in G$ and for any zero submatrix $\left((g . w)_{m, j}\right)_{m \in M, j \in J}$ we have

$$
\sum_{m \in M} \mu_{m}^{\prime}(\leq)<\sum_{j \notin J} \lambda_{j}^{\prime}
$$

For convenience we replace each $\lambda_{i}$ with $\lambda_{i} / d$ and each $\mu_{l}$ with $\mu_{l} / d$. Thus our polarization $\Lambda$ will be a tuple of rational numbers satisfying

$$
\sum_{i=1}^{r} m_{i} \lambda_{i}=1=\sum_{l=1}^{s} n_{l} \mu_{l} .
$$

The set of polarizations can be realized as an open subset of the Euclidean space of dimension $r+s-2$. 


\section{Sheaves $\mathscr{F}$ with $h^{0}(\mathscr{F}(-1))=0$ and $h^{1}(\mathscr{F})=0$}

The main technical tool that we will use in this paper is the Beilinson complex. Given a coherent sheaf $\mathscr{F}$ on $\mathbb{P}^{2}$ there is a sequence of sheaves

$$
0 \rightarrow \mathscr{C}^{-2} \rightarrow \mathscr{C}^{-1} \rightarrow \mathscr{C}^{0} \rightarrow \mathscr{C}^{1} \rightarrow \mathscr{C}^{2} \rightarrow 0
$$

that is exact except in the middle where the cohomology is $\mathscr{F}$. The sheaves $\mathscr{C}^{i}$ are given by

$$
\begin{aligned}
\mathscr{C}^{-2} & =H^{0}\left(\mathscr{F} \otimes \Omega^{2}(2)\right) \otimes \mathcal{O}(-2), \\
\mathscr{C}^{-1} & =H^{0}\left(\mathscr{F} \otimes \Omega^{1}(1)\right) \otimes \mathcal{O}(-1) \oplus H^{1}\left(\mathscr{F} \otimes \Omega^{2}(2)\right) \otimes \mathcal{O}(-2), \\
\mathscr{C}^{0} & =H^{0}(\mathscr{F}) \otimes \mathcal{O} \oplus H^{1}\left(\mathscr{F} \otimes \Omega^{1}(1)\right) \otimes \mathcal{O}(-1) \oplus H^{2}\left(\mathscr{F} \otimes \Omega^{2}(2)\right) \otimes \mathcal{O}(-2), \\
\mathscr{C}^{1} & =H^{1}(\mathscr{F}) \otimes \mathcal{O} \oplus H^{2}\left(\mathscr{F} \otimes \Omega^{1}(1)\right) \otimes \mathcal{O}(-1), \\
\mathscr{C}^{2} & =H^{2}(\mathscr{F}) \otimes \mathscr{O} .
\end{aligned}
$$

The sheaves $\mathscr{F}$ we are interested in are supported on curves, so

$$
H^{2}(\mathscr{F})=0, \quad H^{2}\left(\mathscr{F} \otimes \Omega^{1}(1)\right)=0, \quad H^{2}\left(\mathscr{F} \otimes \Omega^{2}(2)\right)=0 .
$$

Also, on $\mathbb{P}^{2}$ we have $\Omega^{2}(2)=\mathcal{O}(-1)$. The Beilinson sequence that we will use takes the form

$$
0 \rightarrow \mathscr{C}^{-2} \rightarrow \mathscr{C}^{-1} \rightarrow \mathscr{C}^{0} \rightarrow \mathscr{C}^{1} \rightarrow 0
$$

where

$$
\begin{aligned}
\mathscr{C}^{-2} & =H^{0}(\mathscr{F}(-1)) \otimes \mathcal{O}(-2), \\
\mathscr{C}^{-1} & =H^{0}\left(\mathscr{F} \otimes \Omega^{1}(1)\right) \otimes \mathcal{O}(-1) \oplus H^{1}(\mathscr{F}(-1)) \otimes \mathcal{O}(-2), \\
\mathscr{C}^{0} & =H^{0}(\mathscr{F}) \otimes \mathcal{O} \oplus H^{1}\left(\mathscr{F} \otimes \Omega^{1}(1)\right) \otimes \mathscr{O}(-1), \\
\mathscr{C}^{1} & =H^{1}(\mathscr{F}) \otimes \mathcal{O} .
\end{aligned}
$$

The morphisms

$$
\begin{aligned}
H^{0}(\mathscr{F}(-1)) \otimes \mathcal{O}(-2) & \rightarrow H^{1}(\mathscr{F}(-1)) \otimes \mathcal{O}(-2), \\
H^{0}\left(\mathscr{F} \otimes \Omega^{1}(1)\right) \otimes \mathcal{O}(-1) & \rightarrow H^{1}\left(\mathscr{F} \otimes \Omega^{1}(1)\right) \otimes \mathcal{O}(-1), \\
H^{0}(\mathscr{F}) \otimes \mathcal{O} & \rightarrow H^{1}(\mathscr{F}) \otimes \mathcal{O}
\end{aligned}
$$

from above are all zero. Indeed, each of these morphisms can be represented by a matrix with scalar entries. Performing Gaussian elimination on these matrices we arrive at a complex like (4.1) in which the cohomology vector spaces get replaced by subspaces. Using standard methods in cohomology theory we can show that the dimension of each of these subspaces equals the dimension of the corresponding 
cohomology space. In other words no Gaussian elimination was performed; the matrices were zero to begin with.

Apart from the question of the semistability of the morphism $\varphi$, the resolutions of generic sheaves giving points in $\mathrm{M}_{\mathbb{P}^{2}}(r, \chi)$ appeared first in [Freiermuth 2000]. For the sake of completeness we have included the really simple arguments here without quoting every time the above work.

Claim 4.2. Let $\mathscr{F}$ be a sheaf on $\mathbb{P}^{2}$ with $h^{0}(\mathscr{F}(-1))=0$. Let $n \geq 2$ be an integer and assume that $\mathscr{F}_{F}$ has Hilbert polynomial $P_{\mathscr{F}}(t)=(n+1) t+n$. Then $\mathscr{F}$ is semistable if and only if it has a resolution

$$
0 \rightarrow \mathcal{O}(-2) \oplus(n-1) \mathcal{O}(-1) \stackrel{\varphi}{\rightarrow} n \mathcal{O} \rightarrow \mathscr{F} \rightarrow 0
$$

with $\varphi$ not equivalent to a matrix of the form

$$
\left[\begin{array}{ll}
\star & \psi \\
\star & 0
\end{array}\right]
$$

where $\psi: m \mathscr{O}(-1) \rightarrow m \mathbb{O}, 1 \leq m \leq n-1$.

Equivalently, $\mathscr{F}$ is semistable if and only if it has a resolution as above with $\varphi$ semistable with respect to $\Lambda$. Here $\Lambda=\left(\lambda_{1}, \lambda_{2}, \mu_{1}\right)$ is any polarization satisfying $0<\lambda_{1}<1 / n$.

Proof. We have $h^{0}(\mathscr{F}(-1))=0, h^{1}(\mathscr{F}(-1))=1, h^{0}(\mathscr{F})=n, h^{1}(\mathscr{F})=0$ because of Proposition 2.3. Thus (4.1) gives the following resolution with $\varphi_{12}=0$ :

$$
0 \rightarrow \mathcal{O}(-2) \oplus(m+n-1) \mathcal{O}(-1) \stackrel{\varphi}{\rightarrow} m \mathscr{O}(-1) \oplus n \mathcal{O} \rightarrow \mathscr{F} \rightarrow 0 .
$$

Here $m$ is an integer and, since $\varphi$ is injective, we can only have $m=0$ or $m=1$.

Assume that $\mathscr{F}$ is semistable. Then $m \neq 1$, otherwise $\mathscr{F}$ would have a subsheaf $\mathscr{F}^{\prime}$ with resolution

$$
0 \rightarrow n \mathscr{O}(-1) \rightarrow n \mathscr{O} \rightarrow \mathscr{F}^{\prime} \rightarrow 0 .
$$

We have $P_{\mathscr{F}^{\prime}}(t)=n t+n$, hence such a subsheaf would destabilize $\mathscr{F}$. Thus far we have obtained a resolution

$$
0 \rightarrow \mathcal{O}(-2) \oplus(n-1) \mathcal{O}(-1) \stackrel{\varphi}{\rightarrow} n \mathscr{O} \rightarrow \mathscr{F} \rightarrow 0 .
$$

We mention that this resolution was first obtained in [Maican 2000] and it is also present in [Freiermuth 2000]. The matrix $\varphi$ cannot be equivalent to a matrix of the form

$$
\left[\begin{array}{cc}
\star & \psi \\
\varphi_{21} & 0
\end{array}\right]
$$


otherwise we would get an exact commutative diagram in which $\mathscr{F}^{\prime}$ is a destabilizing subsheaf of $\mathscr{F}$.

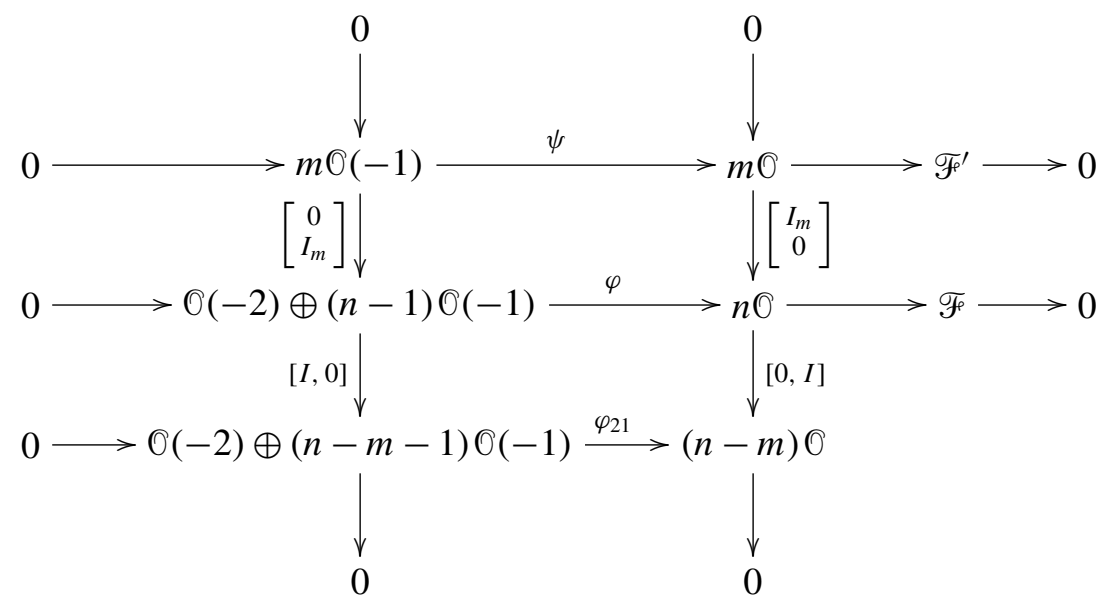

Conversely, we assume that $\mathscr{F}$ has a resolution as in the statement of the claim, and we try to show that the conditions from Remark 2.2 are satisfied. At every point $x$ in the support of $\mathscr{F}$ we have

$$
\operatorname{depth}_{x} \mathscr{F}=2-\operatorname{pd}_{x} \mathscr{F} \geq 1,
$$

showing that $\mathscr{F}$ does not have zero-dimensional torsion. Assume now that $\mathscr{F}$ has a subsheaf $\mathscr{F}^{\prime}$ which contradicts Remark 2.2(ii), in other words which satisfies

$$
\frac{\alpha_{0}\left(\mathscr{F}^{\prime}\right)}{\alpha_{1}\left(\mathscr{F}^{\prime}\right)}>\frac{n}{n+1} .
$$

The multiplicity $m=\alpha_{1}\left(\mathscr{F}^{\prime}\right)$ cannot exceed the multiplicity of $\mathscr{F}$. Thus $\alpha_{0}\left(\mathscr{F}^{\prime}\right) \geq m$. Since $h^{0}\left(\mathscr{F}^{\prime}(-1)\right) \leq h^{0}(\mathscr{F}(-1))=0$ we have

$$
P_{\mathscr{F}^{\prime}}(-1)=-h^{1}\left(\mathscr{F}^{\prime}(-1)\right) \leq 0,
$$

forcing $\alpha_{0}\left(\mathscr{F}^{\prime}\right) \leq m$. So far we have obtained $P_{\mathscr{F}^{\prime}}(t)=m t+m$ for some integer $1 \leq m \leq n$. Now notice that $\mathscr{F}$ is generated by global sections, so we must have $h^{0}\left(\mathscr{F}^{\prime}\right) \leq n-1$, forcing $m \leq n-1$. We have

$$
h^{0}\left(\mathscr{F}^{\prime}(-2)\right)=0, \quad h^{1}\left(\mathscr{F}^{\prime}(-2)\right)=m, \quad h^{0}\left(\mathscr{F}^{\prime}(-1)\right)=0, \quad h^{1}\left(\mathscr{F}^{\prime}(-1)\right)=0 .
$$

The Beilinson sequence of $\mathscr{F}^{\prime}(-1)$ gives a resolution

$$
0 \rightarrow m \mathscr{O}(-2) \rightarrow m \mathscr{O}(-1) \rightarrow \mathscr{F}^{\prime}(-1) \rightarrow 0 .
$$

This yields a commutative diagram 


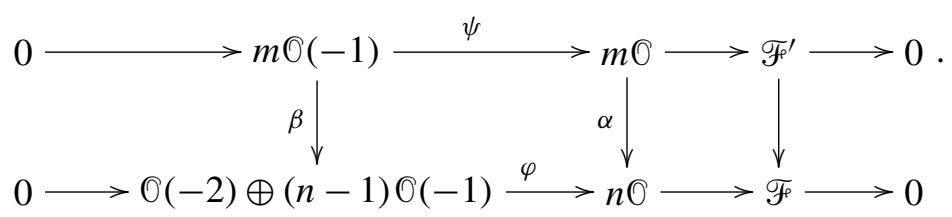

The map $\alpha$ is injective because it is injective on the level of global sections. Hence also $\beta$ is injective. It is clear now that $\varphi$ is equivalent to a matrix of the form

$$
\left[\begin{array}{ll}
\star & \psi \\
\star & 0
\end{array}\right]
$$

This contradicts the hypothesis and finishes the proof of the first part of the claim.

The second part of the claim follows from Proposition 3.3. Namely, King's criterion says that $\varphi$ is semistable with respect to $\Lambda$ if and only if whenever

$$
\varphi \sim\left[\begin{array}{ll}
\star & \psi \\
\star & 0
\end{array}\right] \quad \text { with } \quad \psi: p \mathscr{O}(-2) \oplus q \mathbb{O}(-1) \rightarrow m \mathscr{O}
$$

we have $m \mu_{1} \geq p \lambda_{1}+q \lambda_{2}$. Thus, we need to find $\Lambda$ satisfying the conditions

$$
m \mu_{1}<p \lambda_{1}+q \lambda_{2} \quad \text { if and only if } q \geq m .
$$

Here $0 \leq m \leq n, 0 \leq p \leq 1,0 \leq q \leq n-1$. These conditions are the same as

$$
\begin{aligned}
& m \mu_{1}<m \lambda_{2} \quad \text { for } \quad 1 \leq m \leq n-1 \text {, } \\
& m \mu_{1} \geq \lambda_{1}+(m-1) \lambda_{2} \quad \text { for } \quad 1 \leq m \leq n .
\end{aligned}
$$

Using relations (3.4)

$$
\mu_{1}=\frac{1}{n}, \quad \lambda_{2}=\frac{1-\lambda_{1}}{n-1},
$$

we arrive at the conditions

$$
\lambda_{1}<\frac{1}{n}, \quad \frac{n-m}{n(n-1)} \geq \lambda_{1} \frac{n-m}{n-1} .
$$

The conditions on $\lambda_{1}$ are precisely those of the claim.

Claim 4.3. Let $\mathscr{F}$ be a sheaf on $\mathbb{P}^{2}=\mathbb{P}(V)$ with $h^{0}(\mathscr{F}(-1))=h^{1}(\mathscr{F})=0$. Assume

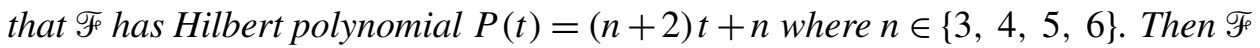
is semistable if and only if it has a resolution

$$
0 \rightarrow 2 \mathcal{O}(-2) \oplus(n-2) \mathcal{O}(-1) \stackrel{\varphi}{\rightarrow} n \mathscr{O} \rightarrow \mathscr{F} \rightarrow 0
$$

with $\varphi$ not equivalent to a matrix of the form

$$
\left[\begin{array}{ll}
\star & \psi \\
\star & 0
\end{array}\right]
$$


where $\psi: m \mathscr{O}(-1) \rightarrow m \mathcal{O}, 1 \leq m \leq n-2$ or $\psi: \mathcal{O}(-2) \oplus(m-1) \mathcal{O}(-1) \rightarrow$ $m 0, n / 2<m \leq n-1$, or it has a resolution

$$
0 \rightarrow 2 \mathcal{O}(-2) \oplus(n-1) \mathcal{O}(-1) \stackrel{\varphi}{\rightarrow} \mathcal{O}(-1) \oplus n \mathscr{O} \rightarrow \mathscr{F} \rightarrow 0
$$

whith $\varphi_{11}$ having linearly independent entries, $\varphi_{12}=0, \varphi_{21} \neq 0$ and $\varphi_{22}$ not equivalent to a matrix of the form

$$
\left[\begin{array}{ll}
\star & \psi \\
\star & 0
\end{array}\right]
$$

where $\psi: m \mathscr{O}(-1) \rightarrow m \mathscr{O}, 1 \leq m \leq n-1$.

The maps $\varphi$ occuring in (i) are precisely those maps $\varphi \in W^{\mathrm{ss}}(G, \Lambda)$ with nonzero determinant. Here $\Lambda=\left(\lambda_{1}, \lambda_{2}, \mu_{1}\right)$ is any polarization satisfying $1 /(2 n) \leq \lambda_{1}<$ $1 / n$.

The maps $\varphi$ occuring in (ii) are precisely those maps $\varphi \in W^{\mathrm{ss}}(G, \Lambda)$ with $\operatorname{det}(\varphi) \neq 0$ and $\varphi_{12}=0$. Here $\Lambda=\left(\lambda_{1}, \lambda_{2}, \mu_{1}, \mu_{2}\right)$ is any polarization for which the pair $\left(\lambda_{1}, \mu_{1}\right)$ is in the interior of the triangle with vertices

$$
(0,0), \quad\left(\frac{1}{n+1}, \frac{1}{n+1}\right), \quad\left(\frac{1}{n^{2}-n+2}, \frac{2}{n^{2}-n+2}\right) .
$$

When $n \geq 7$ solely the "only if" part of the above statement is true. Thus, all we can say in the case $n \geq 7$, is that each semistable sheaf F occurs as the cokernel of a semistable $\varphi$, but there are semistable morphisms $\varphi$ whose cokernel is not a semistable sheaf.

Proof. One direction is clear, cf. the proof of Claim 4.2. Conversely, suppose that $\mathscr{F}^{\prime} \subset \mathscr{F}$ is a destabilizing subsheaf. Arguing as in Claim 4.2 we see that the Hilbert polynomial of $\mathscr{F}^{\prime}$ is either $m t+m$ with $1 \leq m \leq n$ or $(m+1) t+m$ with $n / 2<m \leq n$. In the case $P_{\mathscr{F}^{\prime}}(t)=m t+m$ we deduce that $\varphi$ is equivalent to a matrix of the form

$$
\left[\begin{array}{ll}
\star & \psi \\
\star & 0
\end{array}\right]
$$

where $\psi: m \mathcal{O}(-1) \rightarrow m \mathcal{O}$. Assume now that $P_{\mathscr{F}^{\prime}}(t)=(m+1) t+m$ with $n / 2<$ $m \leq n$. We have

$$
h^{0}\left(\mathscr{F}^{\prime}(-1)\right)=0, \quad h^{1}\left(\mathscr{F}^{\prime}(-1)\right)=1, \quad h^{0}\left(\mathscr{F}^{\prime}(-2)\right)=0, \quad h^{1}\left(\mathscr{F}^{\prime}(-2)\right)=m+2 .
$$

From $\Omega^{1} \subset 30(-1)$ and $h^{0}\left(\mathscr{F}^{\prime}(-1)\right)=0$ we get $h^{0}\left(\mathscr{F}^{\prime} \otimes \Omega^{1}\right)=0$. The Beilinson sequence of $\mathscr{F}^{\prime}(-1)$, which has $\mathscr{F}^{\prime}(-1)$ as middle cohomology, takes the form

$$
0 \rightarrow(m+2) \mathcal{O}(-2) \rightarrow(m+3) \mathcal{O}(-1) \stackrel{\eta}{\rightarrow} \mathrm{O} \rightarrow 0 .
$$

Since $\eta$ is surjective it must be equivalent to a matrix of the form

$$
(X, Y, Z, 0, \ldots, 0) \text {. }
$$


So far we have arrived at the following resolution of $\mathscr{F}^{\prime}$ :

$$
0 \rightarrow(m+2) \mathcal{O}(-1) \rightarrow \Omega^{1}(1) \oplus m \mathscr{O} \rightarrow \mathscr{F}^{\prime} \rightarrow 0 .
$$

From this we get $h^{1}\left(\mathscr{F}^{\prime}\right)=0, h^{0}\left(\mathscr{F}^{\prime}\right)=m$. Writing

$$
p=h^{1}\left(\mathscr{F}^{\prime} \otimes \Omega^{1}(1)\right),
$$

the sequence (4.1) gives the resolution

$$
0 \rightarrow \mathcal{O}(-2) \oplus(m+p-1) \mathcal{O}(-1) \stackrel{\psi}{\rightarrow} p \mathcal{O}(-1) \oplus m \mathcal{O} \rightarrow \mathscr{F}^{\prime} \rightarrow 0,
$$

with $\psi_{12}=0$. From the injectivity of $\psi$ we see that we can only have $p=0$ or $p=1$. In the latter case $\mathscr{F}^{\prime}$ has a subsheaf $\mathscr{F}^{\prime \prime}$ with resolution

$$
0 \rightarrow m \mathcal{O}(-1) \rightarrow m \mathcal{O} \rightarrow \mathscr{F}^{\prime \prime} \rightarrow 0 .
$$

This situation has been examined before. Thus we arrive at the resolution

$$
0 \rightarrow \mathcal{O}(-2) \oplus(m-1) \mathcal{O}(-1) \stackrel{\psi}{\rightarrow} m \mathscr{O} \rightarrow \mathscr{F}^{\prime} \rightarrow 0 .
$$

We get the following exact commutative diagrams in case (i)

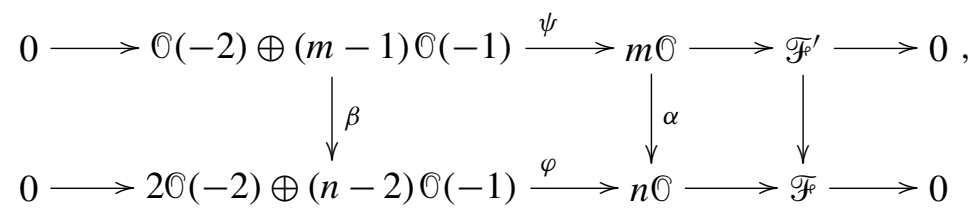

and in case (ii)

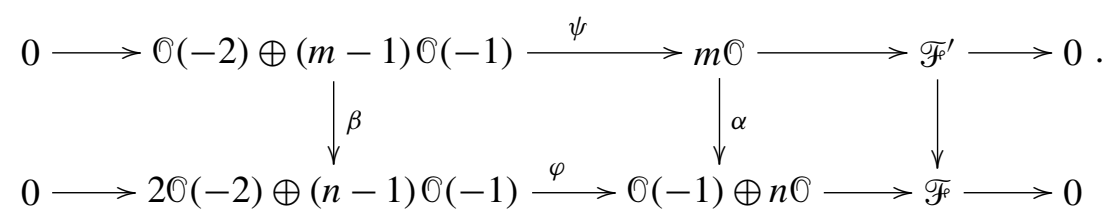

The map $\alpha$ is injective because it is injective on global sections. It follows that $\beta$ is also injective. If $\beta_{11} \neq 0$, which can happen only in case (i), we get the contradiction

$$
\varphi \sim\left[\begin{array}{cc}
\star & \psi \\
\star & 0
\end{array}\right]
$$

In case (ii) we have $\varphi_{11} \beta_{11}=\alpha_{11} \psi_{11}=0$, forcing $\beta_{11}=0$ because, by hypothesis, the entries of $\varphi_{11}$ are linearly independent. Assume from now on that $\beta_{11}=0$. The 
case

$$
\left[\begin{array}{ll}
\beta_{21} & \beta_{22}
\end{array}\right] \sim\left[\begin{array}{cccc}
X & 0 & \cdots & 0 \\
Y & 0 & \cdots & 0 \\
Z & 0 & \cdots & 0 \\
0 & 1 & \cdots & 0 \\
\vdots & \vdots & \ddots & \vdots \\
0 & 0 & \cdots & 1 \\
0 & 0 & \cdots & 0 \\
\vdots & \vdots & & \vdots \\
0 & 0 & \cdots & 0
\end{array}\right]
$$

leads to $n-1 \geq 3+m-1$, so $n \geq 3+m>3+n / 2$, which contradicts the hypothesis $n \leq 6$. The case

$$
\left[\begin{array}{ll}
\beta_{21} & \beta_{22}
\end{array}\right] \sim\left[\begin{array}{cccc}
X & 0 & \cdots & 0 \\
0 & 1 & \cdots & 0 \\
\vdots & \vdots & \ddots & \vdots \\
0 & 0 & \cdots & 1 \\
0 & 0 & \cdots & 0 \\
\vdots & \vdots & & \vdots \\
0 & 0 & \cdots & 0
\end{array}\right]
$$

is excluded from the fact that $\operatorname{Coker}(\beta)$, as a subsheaf of the torsion-free sheaf Goker $(\alpha)$, must be torsion-free. We are left with the case

$$
\left[\begin{array}{ll}
\beta_{21} & \beta_{22}
\end{array}\right] \sim\left[\begin{array}{cccc}
X & 0 & \cdots & 0 \\
Y & 0 & \cdots & 0 \\
0 & 1 & \cdots & 0 \\
\vdots & \vdots & \ddots & \vdots \\
0 & 0 & \cdots & 1
\end{array}\right]
$$

which yields

$$
\varphi \sim\left[\begin{array}{cc}
\star & \psi^{\prime} \\
\star & 0
\end{array}\right]
$$

with $\psi^{\prime}$ an $(m+1) \times(m+1)$-matrix with entries in $V^{*}$. This again contradicts the hypothesis and shows that $\mathscr{F}$ is semistable.

The part of the claim concerning the semistability of $\varphi$ follows from Proposition 3.3. Namely, in case (i), we are looking for $\Lambda$ satisfying 


$$
\begin{array}{ll}
m \mu_{1}<m \lambda_{2} & \text { for } \quad 1 \leq m \leq n-2, \\
m \mu_{1}<\lambda_{1}+(m-1) \lambda_{2} & \text { for } n / 2<m \leq n-1, \\
m \mu_{1} \geq(m-1) \lambda_{2} & \text { for } n / 2<m \leq n-1, \\
m \mu_{1} \geq \lambda_{1}+(m-1) \lambda_{2} & \text { for } \quad 1 \leq m \leq n / 2, \\
m \mu_{1} \geq 2 \lambda_{1}+(m-2) \lambda_{2} & \text { for } \quad 2 \leq m \leq n .
\end{array}
$$

Using relations (3.4) the above conditions become $1 /(2 n) \leq \lambda_{1}<1 / n$. Similarly, in case (ii), we need to find $\Lambda$ satisfying the conditions

$$
\begin{aligned}
\mu_{1} & <2 \lambda_{1}, & & \\
m \mu_{2} & <m \lambda_{2} & & \text { for } 1 \leq m \leq n-1, \\
\mu_{1} & >\lambda_{1}, & & \\
m \mu_{2} & \geq(m-1) \lambda_{2} & & \text { for } 1 \leq m \leq n, \\
\mu_{1}+m \mu_{2} & <\lambda_{1}+m \lambda_{2} & & \text { for } 1 \leq m \leq n-1, \\
\mu_{1}+m \mu_{2} & \geq 2 \lambda_{1}+(m-1) \lambda_{2} & & \text { for } 1 \leq m \leq n .
\end{aligned}
$$

Using (3.4) the above conditions can be translated into the conditition that $\left(\lambda_{1}, \mu_{1}\right)$ is in the interior of a triangle as in the statement of the claim.

Observation 4.4. If $n \geq 7$ then the situation

$$
\left[\begin{array}{ll}
\beta_{21} & \beta_{22}
\end{array}\right] \sim \beta_{0}=\left[\begin{array}{cccc}
X & 0 & \cdots & 0 \\
Y & 0 & \cdots & 0 \\
Z & 0 & \cdots & 0 \\
0 & 1 & \cdots & 0 \\
\vdots & \vdots & \ddots & \vdots \\
0 & 0 & \cdots & 1 \\
0 & 0 & \cdots & 0 \\
\vdots & \vdots & & \vdots \\
0 & 0 & \cdots & 0
\end{array}\right]
$$

is feasible. Thus, to ensure the semistability of $\mathscr{F}$, we would have to exclude, say in case (i), matrices of the form

$$
\left[\begin{array}{ccc}
\star & \star & \psi^{\prime \prime} \\
\star & \psi^{\prime} & 0 \\
\star & 0 & 0
\end{array}\right] \quad \text { with } \quad \psi^{\prime} \sim\left[\begin{array}{ccc}
Y & X & 0 \\
Z & 0 & X \\
0 & -Z & Y
\end{array}\right]
$$

and $\psi^{\prime \prime}$ an $m \times(m-1)$-matrix with entries in $V^{*}$. From Proposition 3.3 we see that such conditions cannot be formulated in terms of semistability, so they are beyond the interest of this paper. Indeed, according to Proposition 3.3, semistability 
conditions on a matrix specify that the matrix should not, up to equivalence, have certain zero submatrices.

Claim 4.5. Let $\mathscr{F}$ be a sheaf on $\mathbb{P}^{2}=\mathbb{P}(V)$ with $h^{0}(\mathscr{F}(-1))=h^{1}(\mathscr{F})=0$. Assume that $\mathscr{F}$ has Hilbert polynomial $P(t)=4 t+2$. Then $\mathscr{F}$ is semistable if and only if it has a resolution

$$
0 \rightarrow 20(-2) \rightarrow 20 \rightarrow \mathscr{F} \rightarrow 0
$$

or it has a resolution

$$
0 \rightarrow 2 \mathrm{O}(-2) \oplus \mathcal{O}(-1) \stackrel{\varphi}{\rightarrow} \mathrm{O}(-1) \oplus 2 \mathrm{O} \rightarrow \mathscr{F} \rightarrow 0
$$

with

$$
\varphi=\left[\begin{array}{ccc}
X_{1} & X_{2} & 0 \\
\star & \star & Y_{1} \\
\star & \star & Y_{2}
\end{array}\right],
$$

where $X_{1}, X_{2} \in V^{*}$ are linearly independent one-forms and, likewise, $Y_{1}, Y_{2} \in$ $V^{*}$ are linearly independent. These morphisms are precisely the morphisms $\varphi \in$ $W^{\mathrm{ss}}(G, \Lambda)$ with nonzero determinant and $\varphi_{12}=0$. Here

$$
\Lambda=\left(\lambda_{1}, \lambda_{2}, \mu_{1}, \mu_{2}\right)
$$

is any polarization for which the pair $\left(\lambda_{1}, \mu_{1}\right)$ belongs to the interior of the quadrilateral with vertices

$$
(0,0), \quad\left(\frac{1}{3}, \frac{1}{3}\right), \quad\left(\frac{1}{2}, 1\right), \quad(0,1) .
$$

Proof. For the first part of the claim the proof is the same as the proof of Claim 4.3 so we omit it. For the part of the claim concerning the semistability of $\varphi$ we arrive at the following conditions on $\Lambda$ :

$$
\mu_{1}>\lambda_{1} \text { and } \mu_{2}<\lambda_{2} \text { which is the same as } \mu_{1}>4 \lambda_{1}-1 .
$$

They describe the quadrilateral from the claim.

In the remaining part of this section we will be concerned with sheaves $\mathscr{F}$ on $\mathbb{P}^{2}$ having Hilbert polynomial $P(t)=(n+3) t+n, n \geq 3$, and satisfying $h^{0}(\mathscr{F}(-1))=$ $0, h^{1}(\mathscr{F})=0$. Such a sheaf $\mathscr{F}$ has one of the following resolutions:

$$
\begin{gathered}
0 \rightarrow 3 \mathcal{O}(-2) \oplus(n-3) \mathcal{O}(-1) \stackrel{\varphi}{\rightarrow} n \mathscr{O} \rightarrow \mathscr{F} \rightarrow 0, \\
0 \rightarrow 3 \mathcal{O}(-2) \oplus(n-2) \mathcal{O}(-1) \stackrel{\varphi}{\rightarrow} \mathcal{O}(-1) \oplus n \mathcal{O} \rightarrow \mathscr{F} \rightarrow 0, \\
0 \rightarrow 3 \mathcal{O}(-2) \oplus(n-1) \mathcal{O}(-1) \stackrel{\varphi}{\rightarrow} 2 \mathcal{O}(-1) \oplus n \mathcal{O} \rightarrow \mathscr{F} \rightarrow 0,
\end{gathered}
$$

with $\varphi_{12}=0$ in cases (ii) and (iii). 
Claim 4.6. Let $\mathscr{F}$ be a sheaf with resolution (i) and $3 \leq n \leq 6$. Then $\mathscr{F}$ is semistable if and only if $\varphi$ is not equivalent to a matrix of the form

$$
\left[\begin{array}{ll}
\star & \psi \\
\star & 0
\end{array}\right]
$$

where

$$
\begin{array}{lrl}
\psi: m \mathscr{O}(-1) \rightarrow m \mathscr{O}, & 1 \leq m \leq n-3, & \text { or } \\
\psi: \mathcal{O}(-2) \oplus(m-1) \mathcal{O}(-1) \rightarrow m \mathscr{O}, & n / 3<m \leq n-2, & \text { or } \\
\psi: 2 \mathcal{O}(-2) \oplus(m-2) \mathcal{O}(-1) \rightarrow m \mathcal{O}, & 2 n / 3<m \leq n-1 .
\end{array}
$$

Thus, any sheaf $\mathscr{F}$ with Hilbert polynomial $6 t+3$ and resolution

$$
0 \rightarrow 30(-2) \stackrel{\varphi}{\rightarrow} 30 \rightarrow \mathscr{F} \rightarrow 0
$$

is semistable. The morphisms $\varphi$ occuring form the open subset of $W^{\mathrm{ss}}(G, \Lambda)$ given by the condition $\operatorname{det}(\varphi) \neq 0$. Here $\Lambda$ is the only admissible polarization, namely $\Lambda=\left(\frac{1}{3}, \frac{1}{3}\right)$.

A sheaf $\mathscr{F}$ with Hilbert polynomial $(n+3) t+n, n \in\{4,5,6\}$, and resolution

$$
0 \rightarrow 3 \mathfrak{O}(-2) \oplus(n-3) \mathcal{O}(-1) \stackrel{\varphi}{\rightarrow} n \mathscr{O} \rightarrow \mathscr{F} \rightarrow 0
$$

is semistable if and only if $\varphi$ is semistable with respect to any polarization $\Lambda=$ $\left(\lambda_{1}, \lambda_{2}, \mu_{1}\right)$ satisfying $2 /(3 n) \leq \lambda_{1}<1 / n$.

Proof. One direction is clear. For the other direction suppose that $\mathscr{F}^{\prime} \subset \mathscr{F}_{F}$ is a destabilizing subsheaf. The Hilbert polynomial of $\mathscr{F}^{\prime}$ must be one of the following: $m t+m$, with $1 \leq m \leq n,(m+1) t+m$ with $\frac{n}{3}<m \leq n,(m+2) t+m$ with $\frac{2 n}{3}<m \leq n$.

In the case $P_{\mathscr{F F}^{\prime}}(t)=m t+m$ we deduce, as in the proof of Claim 4.2, that

$$
\varphi \sim\left[\begin{array}{ll}
\star & \psi \\
\star & 0
\end{array}\right] \quad \text { with } \quad \psi: m \mathscr{O}(-1) \rightarrow m \mathcal{O}
$$

In the case $P_{\mathscr{F}^{\prime}}(t)=(m+1) t+m$ we arrive, as in the proof of Claim 4.3, at

$$
\varphi \sim\left[\begin{array}{ll}
\star & \psi \\
\star & 0
\end{array}\right]
$$

with $\psi: \mathbb{O}(-2) \oplus(m-1) \mathscr{O}(-1) \rightarrow m \mathbb{O}$ or $\psi:(m+1) \mathscr{O}(-1) \rightarrow(m+1) \mathbb{O}$.

Assume now that $P_{\mathscr{F}^{\prime}}(t)=(m+2) t+m$ with $\frac{2 n}{3}<m \leq n$. Since $\mathscr{F}_{F}$ is generated by global sections we must have $h^{0}\left(\mathscr{F}^{\prime}\right) \leq n-1$. Thus $n-1 \geq m+h^{1}\left(\mathscr{F}^{\prime}\right)>\frac{2 n}{3}+h^{1}\left(\mathscr{F}^{\prime}\right), \quad \frac{n}{3}-1>h^{1}\left(\mathscr{F}^{\prime}\right), \quad$ forcing $\quad h^{1}\left(\mathscr{F}^{\prime}\right)=0$. 
The Beilinson sequence (4.1) of $\mathscr{F}^{\prime}$ gives one of the following resolutions:

$$
\begin{gathered}
0 \rightarrow 20(-2) \oplus(m-2) \mathcal{O}(-1) \rightarrow m \mathscr{O} \rightarrow \mathscr{F}^{\prime} \rightarrow 0, \\
0 \rightarrow 2 \mathcal{O}(-2) \oplus(m-1) \mathcal{O}(-1) \stackrel{\psi}{\rightarrow} \mathcal{O}(-1) \oplus m \mathcal{O} \rightarrow \mathscr{F}^{\prime} \rightarrow 0, \\
0 \rightarrow 20(-2) \oplus m \mathcal{O}(-1) \stackrel{\psi}{\rightarrow} 2 \mathcal{O}(-1) \oplus m \mathcal{O} \rightarrow \mathscr{F}^{\prime} \rightarrow 0,
\end{gathered}
$$

with $\psi_{12}=0$. In the third case $\mathscr{F}^{\prime}$ has a subsheaf $\mathscr{F}^{\prime \prime}$ with resolution

$$
0 \rightarrow m \mathscr{O}(-1) \rightarrow m \mathscr{O} \rightarrow \mathscr{F}^{\prime \prime} \rightarrow 0 .
$$

This situation has been examined before. In the first case we get an exact commutative diagram

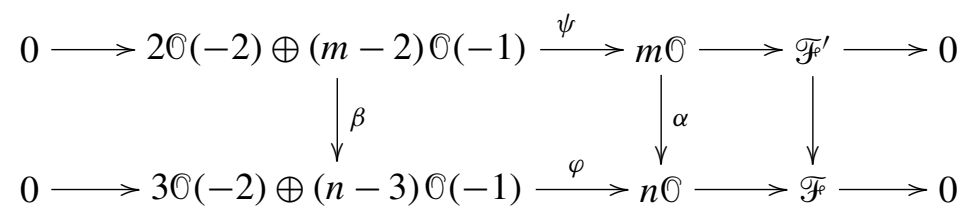

with $\alpha, \beta$ injective. We have $m-2 \leq n-3$ because $\beta_{22}$ is injective. On the other hand, $m \geq n-1$ by hypothesis. Thus $\beta_{22}$ is an isomorphism, forcing $\operatorname{rank}\left(\beta_{11}\right)=2$. In consequence

$$
\varphi \sim\left[\begin{array}{cc}
\star & \psi \\
\star & 0
\end{array}\right]
$$

Finally, assume that $\mathscr{F}^{\prime}$ has the second resolution. We get the commutative diagram

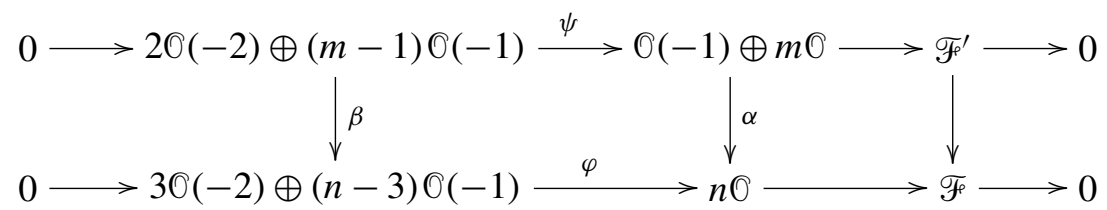

The map $\alpha_{12}$ is injective because $\alpha$ is injective on global sections. We have $\varphi_{12} \beta_{22}=\alpha_{12} \psi_{22}$. The second map is injective, hence $\beta_{22}$ is injective, too. Thus $n-3 \geq m-1, n \geq m+2>\frac{2 n}{3}+2, n>6$, contradiction.

The rest of the proof is as in Claim 4.3, so it will be omitted.

Claim 4.7. Let $\mathscr{F}$ be a sheaf on $\mathbb{P}^{2}=\mathbb{P}(V)$ with resolution (ii) and $3 \leq n \leq 6$. Then $\mathscr{F}$ is semistable if and only if the entries of $\varphi_{11}$ span a subspace of $V^{*}$ of dimension at least two and $\varphi$ is not equivalent to a matrix of the form

$$
\left[\begin{array}{ll}
\star & \psi \\
\star & 0
\end{array}\right],
$$


where

$$
\begin{array}{lrl}
\psi: m \mathscr{O}(-1) \rightarrow m \mathscr{O}, & 1 \leq m \leq n-2, & \text { or } \\
\psi: \mathcal{O}(-2) \oplus(m-1) \mathcal{O}(-1) \rightarrow m \mathscr{O}, & n / 3<m \leq n-1, & \text { or } \\
\psi: 2 \mathcal{O}(-2) \oplus(m-1) \mathcal{O}(-1) \rightarrow \mathcal{O}(-1) \oplus m \mathcal{O}, & 2 n / 3<m \leq n-1 .
\end{array}
$$

Equivalently, $\mathscr{F}$ is semistable if and only if $\varphi$ is semistable with respect to $\Lambda$. Here $\Lambda=\left(\lambda_{1}, \lambda_{2}, \mu_{1}, \mu_{2}\right)$ is a polarization satisfying the property that the pair $\left(\lambda_{1}, \mu_{1}\right)$

(i) is in the interior of the segment with endpoints $\left(\frac{1}{4}, \frac{1}{4}\right)$ and $\left(\frac{1}{5}, \frac{2}{5}\right)$, in the case $n=3$;

(ii) is in the interior of the triangle bounded by the lines $\mu_{1}=\lambda_{1}, \mu_{1}=1-$ $4 \lambda_{1}, \mu_{1}=4 \lambda_{1}-\frac{1}{3}$, in the case $n=4$;

(iii) is in the interior of the triangle bounded by the lines $\mu_{1}=1-5 \lambda_{1}, \mu_{1}=$ $\frac{1}{6}, \mu_{1}=\frac{15}{4} \lambda_{1}-\frac{1}{4}$, in the case $n=5 ;$

(iv) is in the interior of the segment with endpoints $\left(\frac{1}{7}, \frac{1}{7}\right)$ and $\left(\frac{3}{29}, \frac{5}{29}\right)$, in the case $n=6$.

Proof. The proof is the same as the proof of Claim 4.6 with the only difference that when $n \neq 3$ it is possible to have $h^{1}\left(\mathscr{F}^{\prime}\right)=1$ and $P_{\mathscr{F}^{\prime}}(t)=(m+2) t+m$. For such a sheaf the Beilinson sequence takes the form

$$
0 \rightarrow 2 \mathfrak{O}(-2) \oplus(p+m-2) \mathscr{O}(-1) \stackrel{\rho}{\rightarrow} p \mathscr{O}(-1) \oplus(m+1) \mathscr{O} \stackrel{\eta}{\rightarrow} \mathfrak{O} \rightarrow 0 .
$$

As $\eta_{12}=0$ we may assume that $\eta=(X, Y, Z, 0, \ldots, 0)$. Thus $p \geq 3$ and $\mathscr{F}^{\prime}$ has resolution

$0 \rightarrow 2 \mathfrak{O}(-2) \oplus(p+m-2) \mathcal{O}(-1) \rightarrow \Omega^{1} \oplus(p-3) \mathcal{O}(-1) \oplus(m+1) \mathcal{O} \rightarrow \mathscr{F}^{\prime} \rightarrow 0$ from which we get the resolution

$$
\begin{aligned}
0 \rightarrow \mathrm{O}(-3) \oplus 2 \mathcal{O}(-2) \oplus(p+ & m-2) \mathcal{O}(-1) \\
& \stackrel{\varphi^{\prime}}{\rightarrow} 3 \mathcal{O}(-2) \oplus(p-3) \mathcal{O}(-1) \oplus(m+1) \mathcal{O} \rightarrow \mathscr{F}^{\prime} \rightarrow 0
\end{aligned}
$$

with $\varphi_{13}^{\prime}=0$ and $\varphi_{23}^{\prime}=0$. Since $\varphi^{\prime}$ is injective we must have $p=3$. But then $\mathscr{F}^{\prime}$ has a subsheaf $\mathscr{F}^{\prime \prime}$ with resolution

$$
0 \rightarrow(m+1) \mathcal{O}(-1) \rightarrow(m+1) \mathcal{O} \rightarrow \mathscr{F}^{\prime \prime} \rightarrow 0 .
$$

This situation has already been examined.

Claim 4.8. Let $\mathscr{F}$ be a sheaf on $\mathbb{P}^{2}$ with Hilbert polynomial $P_{\mathscr{F}}(t)=6 t+3$ and resolution

$$
0 \rightarrow 30(-2) \oplus 20(-1) \stackrel{\varphi}{\rightarrow} 20(-1) \oplus 30 \rightarrow \mathscr{F} \rightarrow 0,
$$


with $\varphi_{12}=0$. Then $\mathscr{F}$ is semistable if and only if

$$
\varphi_{11} \nsim\left[\begin{array}{ccc}
\star & \star & 0 \\
\star \star & 0
\end{array}\right], \quad \varphi_{11} \nsim\left[\begin{array}{ccc}
\star & 0 & 0 \\
\star \star & \star
\end{array}\right], \quad \varphi_{22} \nsim\left[\begin{array}{cc}
\star & 0 \\
\star & 0 \\
\star & \star
\end{array}\right], \quad \varphi_{22} \nsim\left[\begin{array}{cc}
0 & 0 \\
\star & \star \\
\star & \star
\end{array}\right] .
$$

Equivalently, $\mathscr{F}$ is semistable if and only if $\varphi$ is semistable with respect to $\Lambda$, where $\Lambda=\left(\lambda_{1}, \lambda_{2}, \mu_{1}, \mu_{2}\right)$ is any polarization for which $\left(\lambda_{1}, \mu_{1}\right)$ is in the interior of the triangle with vertices $(0,0),\left(\frac{1}{5}, \frac{1}{5}\right),\left(\frac{1}{3}, \frac{1}{2}\right)$.

Claim 4.9. Let $\mathscr{F}$ be a sheaf on $\mathbb{P}^{2}$ with Hilbert polynomial $P_{\mathscr{F}}(t)=7 t+4$ and resolution

$$
0 \rightarrow 30(-2) \oplus 30(-1) \rightarrow 20(-1) \oplus 40 \rightarrow \mathscr{F} \rightarrow 0,
$$

with $\varphi_{12}=0$. Then $\mathscr{F}$ is semistable if and only if $\varphi_{11}$ satisfies the same conditions as in Claim 4.8 and, in addition, $\varphi_{22}$ is not equivalent to a matrix of the form

$$
\left[\begin{array}{ll}
\star & \psi \\
\star & 0
\end{array}\right] \quad \text { with } \quad \psi: m \mathbb{O}(-1) \rightarrow m \mathbb{O}, \quad m=1,2,3 .
$$

Equivalently, $\mathscr{F}$ is semistable if and only if $\varphi$ is semistable with respect to $\Lambda$, where $\Lambda=\left(\lambda_{1}, \lambda_{2}, \mu_{1}, \mu_{2}\right)$ is any polarization for which $\left(\lambda_{1}, \mu_{1}\right)$ is in the interior of the quadrilateral with vertices $(0,0),\left(\frac{1}{3}, \frac{1}{2}\right),\left(\frac{17}{24}, 1\right),(1,1)$.

\section{Sheaves $\mathscr{F}$ with $h^{0}(\mathscr{F}(-1))=0$ and $h^{1}(\mathscr{F})=1$}

In this section $\mathscr{F}$ will be a sheaf on $\mathbb{P}^{2}$ with $h^{0}(\mathscr{F}(-1))=0, h^{1}(\mathscr{F})=1$ and Hilbert polynomial $P_{\mathscr{F}}(t)=a t+b, 0 \leq b<a$. From the Beilinson complex (4.1) we deduce that $\mathscr{F}$ must have one of the following resolutions:

(i) when $a \leq 2 b$,

$0 \rightarrow(a-b) \mathcal{O}(-2) \oplus(m+2 b-a) \mathcal{O}(-1) \stackrel{\rho}{\rightarrow} \Omega^{1} \oplus(m-3) \mathcal{O}(-1) \oplus(b+1) \mathcal{O} \rightarrow \mathscr{F} \rightarrow 0$,

(ii) when $a>2 b$,

$0 \rightarrow(a-b) \mathcal{O}(-2) \oplus m \mathcal{O}(-1) \stackrel{\rho}{\rightarrow} \Omega^{1} \oplus(m+a-2 b-3) \mathcal{O}(-1) \oplus(b+1) \mathcal{O} \rightarrow \mathscr{F} \rightarrow 0$, where $m$ is an integer and $\rho_{12}=\rho_{22}=0$. Combining these with the exact sequence

$$
0 \rightarrow \mathrm{O}(-3) \rightarrow 30(-2) \rightarrow \Omega^{1} \rightarrow 0
$$

we get one of the following resolutions for $\mathscr{F}$ :

(i) $0 \rightarrow \mathscr{O}(-3) \oplus(a-b) \mathscr{O}(-2) \oplus(m+2 b-a) \mathscr{O}(-1)$

$$
\stackrel{\psi}{\rightarrow} 3 \mathcal{O}(-2) \oplus(m-3) \mathcal{O}(-1) \oplus(b+1) \mathcal{O} \rightarrow \mathscr{F} \rightarrow 0,
$$


(ii)

$$
\begin{aligned}
0 \rightarrow \mathscr{O}(-3) \oplus & (a-b) \mathcal{O}(-2) \oplus m \mathscr{O}(-1) \\
& \stackrel{\psi}{\rightarrow} 3 \mathcal{O}(-2) \oplus(m+a-2 b-3) \mathcal{O}(-1) \oplus(b+1) \mathcal{O} \rightarrow \mathscr{F} \rightarrow 0,
\end{aligned}
$$

where

$$
\psi=\left[\begin{array}{ccc}
X & & \\
Y & \psi_{12} & 0 \\
Z & & \\
0 & \rho_{21} & 0 \\
0 & \rho_{31} & \rho_{32}
\end{array}\right]
$$

Claim 5.1. There are no semistable sheaves $\mathscr{F}$ on $\mathbb{P}^{2}$ with $h^{0}(\mathscr{F}(-1))=0, h^{1}(\mathscr{F})=$ 1 and Hilbert polynomial

$$
P_{\mathscr{F}}(t)=(n+1) t+n \quad \text { or } \quad P_{\mathscr{F}}(t)=(n+2) t+n, \quad n \geq 0 .
$$

Proof. If $a-b=1$ then $\psi$ cannot be injective. If $a-b=2$, then we must have $m=3$ in case (i), respectively $m=2 b+3-a$ in case (ii). It follows that $\mathscr{F}_{F}$ has a subsheaf $\mathscr{F}^{\prime}$ with resolution

$$
0 \rightarrow(n+1) \mathcal{O}(-1) \stackrel{\rho_{32}}{\rightarrow}(n+1) \mathcal{O} \rightarrow \mathscr{F}^{\prime} \rightarrow 0 .
$$

This subsheaf destabilizes $\mathscr{F}$.

If $P_{\mathscr{F}}(t)=(n+1) t+n$, the claim already follows from the fact that $h^{0}(\mathscr{F}(-1))=$ 0 implies $h^{1}(\mathscr{F})=0$; see Proposition 2.3(v). Above we have an alternate argument. ${ }^{1}$

In the remaining part of this section we will assume that $\mathscr{F}$ is a semistable sheaf with Hilbert polynomial $P_{\mathscr{F}}(t)=(n+3) t+n, n \geq 0$. We have the resolution

$0 \rightarrow \mathcal{O}(-3) \oplus 3 \mathcal{O}(-2) \oplus(m+n) \mathcal{O}(-1) \stackrel{\psi}{\rightarrow} 3 \mathcal{O}(-2) \oplus m \mathcal{O}(-1) \oplus(n+1) \mathcal{O} \rightarrow \mathscr{F} \rightarrow 0$.

We must have $m \leq 1$ to ensure that $\psi$ be injective. But if $m=1$ then $\mathscr{F}$ has a destabilizing sheaf $\mathscr{F}^{\prime}$ as above. Thus $m=0$ and we arrive at the resolution

$$
0 \rightarrow \mathcal{O}(-3) \oplus 3 \mathcal{O}(-2) \oplus n \mathcal{O}(-1) \stackrel{\psi}{\rightarrow} 3 \mathcal{O}(-2) \oplus(n+1) \mathcal{O} \rightarrow \mathscr{F} \rightarrow 0 .
$$

\footnotetext{
${ }^{1}$ The referee pointed out that for any sheaf on $\mathbb{P}^{2}$, semistable or not, with Hilbert polynomial $P_{\mathscr{F}}(t)=(n+1) t+n$ and $h^{0}(\mathscr{F}(-1))=0$, we must have $h^{1}(\mathscr{F})=0$. Indeed, as in the proof of Claim 4.3 with $\mathscr{F}$ instead of $\mathscr{F}^{\prime}$, we have a monad

$$
0 \rightarrow(n+2) \mathcal{O}(-1) \rightarrow(n+3) \stackrel{\eta}{\rightarrow} \mathfrak{O}(1) \rightarrow 0
$$

with cohomology $\mathscr{F}$. As $(n+3) \mathcal{O}$ is generated by global sections, it follows that $\mathscr{O}(1)$ is generated by their images under $\eta$. But $O(1)$ cannot be generated by fewer than 3 linearly independent sections. Thus $\eta$ is surjective on the level of global sections. We get $h^{1}(\mathscr{K e r}(\eta))=0$ and, a fortiori, $h^{1}(\mathscr{F})=0$.
} 
Since $\psi$ is injective we must have $\operatorname{rank}\left(\psi_{12}\right) \geq 2$. If $\operatorname{rank}\left(\psi_{12}\right)=2$ then $\mathscr{F}$ has a destabilizing subsheaf $\mathscr{F}^{\prime}$ with resolution

$$
0 \rightarrow \mathfrak{O}(-2) \oplus n \mathscr{O}(-1) \rightarrow(n+1) \mathcal{O} \rightarrow \mathscr{F}^{\prime} \rightarrow 0 .
$$

In conclusion $\operatorname{rank}\left(\psi_{12}\right)=3$ and $\mathscr{F}$ has the resolution

$$
0 \rightarrow \mathcal{O}(-3) \oplus n \mathcal{O}(-1) \stackrel{\varphi}{\rightarrow}(n+1) \mathcal{O} \rightarrow \mathscr{F} \rightarrow 0 .
$$

If $n>3$ some of the semistability conditions on $\mathscr{F}$ cannot be translated into semistability conditions on $\varphi$ because one of the conditions on $\varphi$ would have to be that there is no commutative exact diagram

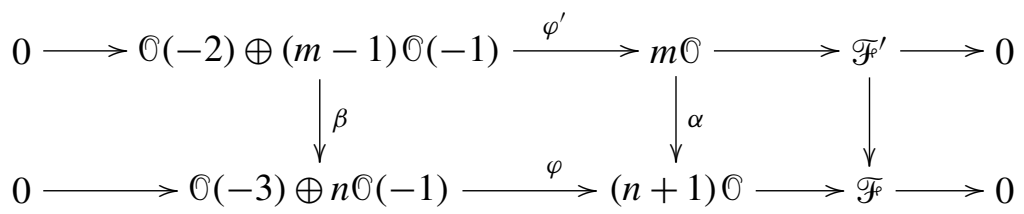

with $\alpha, \beta$ injective and $3 m>n$. If $n>3$ then $\beta$ may have the form

$$
\left[\begin{array}{c}
0 \\
\beta_{0}
\end{array}\right]
$$

with $\beta_{0}$ as in Observation 4.4. In this case the condition $\varphi \beta=\alpha \varphi^{\prime}$ cannot be translated in terms of semistability of $\varphi$.

Claim 5.3. Let $\mathscr{F}$ be a sheaf on $\mathbb{P}^{2}$ with $h^{0}(\mathscr{F}(-1))=0, h^{1}(\mathscr{F})=1$ and Hilbert polynomial

$$
P_{\mathscr{F}}(t)=(n+3) t+n, \quad n=1,2,3 .
$$

Then $\mathscr{F}$ is semistable if and only if it has a resolution

$$
0 \rightarrow \mathcal{O}(-3) \oplus n \mathscr{O}(-1) \stackrel{\varphi}{\rightarrow}(n+1) \mathcal{O} \rightarrow \mathscr{F} \rightarrow 0
$$

with $\varphi$ not equivalent to a matrix of the form

$$
\left[\begin{array}{ll}
\star & \psi \\
\star & 0
\end{array}\right] \quad \text { where } \quad \psi: m \mathscr{O}(-1) \rightarrow m \mathscr{O}, \quad 1 \leq m \leq n .
$$

The morphisms $\varphi$ occuring above are precisely those morphisms semistable with respect to $\Lambda$ with nonzero determinant. Here $\Lambda=\left(\lambda_{1}, \lambda_{2}, \mu_{1}\right)$ is any polarization satisfying $0<\lambda_{1}<1 /(n+1)$. If $n>3$ solely the "only if" part of the above statement remains true.

Proof. One direction follows from the discussion before the claim. Conversely, we assume that $\mathscr{F}$ has a resolution as above and we try to prove that $\mathscr{F}$ is semistable. As in the proof of Claim 4.6, a destabilizing subsheaf $\mathscr{F}^{\prime}$ of $\mathscr{F}$ must have one of 
the following Hilbert polynomials: $m t+m$ with $1 \leq m \leq n,(m+1) t+m$ with $\frac{n}{3}<m \leq n,(m+2) t+m$ with $\frac{2 n}{3}<m \leq n$. In the first case we get the contradiction

$$
\varphi \sim\left[\begin{array}{ll}
\star & \psi \\
\star & 0
\end{array}\right] \quad \text { with } \quad \psi: m \mathscr{O}(-1) \rightarrow m \mathscr{O}, \quad 1 \leq m \leq n
$$

In the second case we have the exact commutative diagram (5.2) with injective $\alpha$ and $\beta$. Since $\operatorname{Coker}(\beta)$ is torsion-free as a subsheaf of the torsion-free sheaf Coker $(\alpha)$, and since

$$
\beta \nsim\left[\begin{array}{c}
0 \\
\beta_{0}
\end{array}\right], \quad \text { we must have } \beta=\left[\begin{array}{c}
0 \\
X \\
Y
\end{array}\right] \text { or } \beta=\left[\begin{array}{ll}
0 & 0 \\
X & 0 \\
Y & 0 \\
0 & 1
\end{array}\right] .
$$

We get

$$
\varphi \sim\left[\begin{array}{ll}
\star & \psi \\
\star & 0
\end{array}\right] \quad \text { with } \quad \psi:(m+1) \mathbb{O}(-1) \rightarrow(m+1) \mathbb{O} .
$$

Finally, in the third case, we must have $m=n$ and, as in the proof of Claim 4.6, an exact commutative diagram

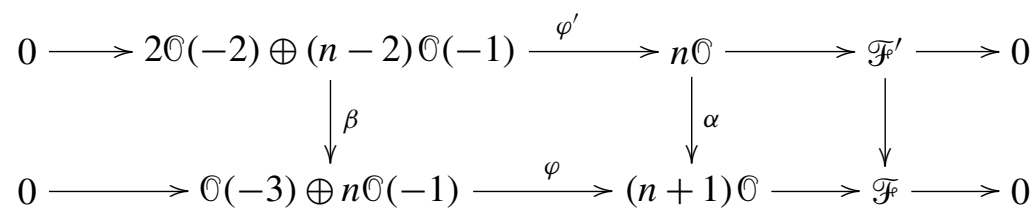

or a diagram

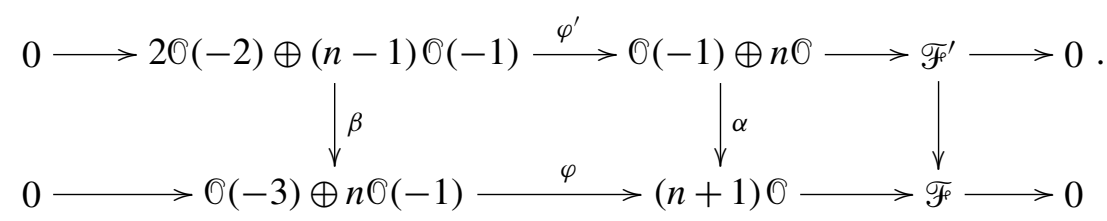

In the first case $\alpha$ is injective because it is injective on global sections. Thus $\beta$ is injective. But then $\mathscr{C o k e r}(\beta)$ has a direct summand supported on a conic. This contradicts the fact that $\mathscr{C o k e r}(\beta)$ is a subsheaf of $\mathscr{C o k e r}(\alpha) \simeq \mathbb{0}$.

In the second diagram we have $\varphi_{12} \beta_{22}=\alpha_{12} \varphi_{22}^{\prime}$. But $\alpha_{12}$ is injective because $\alpha$ is injective on global sections. Also, $\varphi_{22}^{\prime}$ is injective because $\varphi_{12}^{\prime}=0$ and $\varphi^{\prime}$ is injective. Thus $\varphi_{12} \beta_{22}$ is injective, forcing $\beta_{22}$ to be injective. It follows that $\operatorname{Fer}(\beta) \subset 20(-2)$. If $\alpha$ is not injective then we get the contradiction

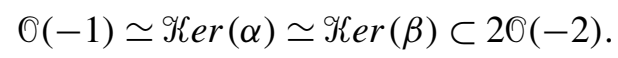

Thus $\alpha$ is injective, forcing $\mathscr{C o k e r}(\alpha)$ to be supported on a line. But this is impossible, because $\mathcal{O}(-3) \subset$ Coker $(\beta) \subset \mathscr{C o k e r}(\alpha)$. 
Remark 5.4. The sheaves from Claim 5.3 with Hilbert polynomial $6 t+3$ are stable. Indeed, assume that $\mathscr{F}$ has a subsheaf $\mathscr{F}^{\prime}$ with Hilbert polynomial $2 t+1$. It must be stable, hence the structure sheaf of a conic. We arrive at a commutative diagram

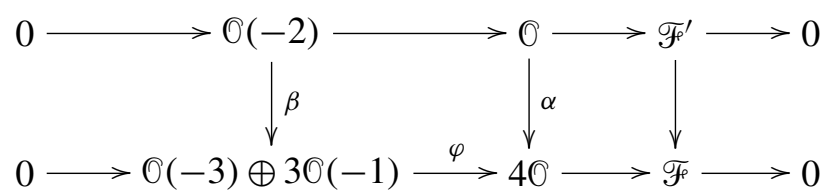

with injective $\alpha$ and $\beta$. After performing column operations on $\varphi$ we may assume that three among the rows of $\varphi_{12} \beta_{21}$ are zero. But, according to Claim 5.3, $\varphi_{12}$ is semistable with respect to the only admissible polarization on the vector space of morphisms $30(-1) \rightarrow 40$. From remark Remark 5.5 we get $\beta_{21}=0$, so $\beta=0$, contradiction.

Assume now that $\mathscr{F}$ has a quotient sheaf $\mathscr{F}^{\prime \prime}=\mathscr{F} / \mathscr{F}^{\prime}$ with Hilbert polynomial $2 t+1$. $\mathscr{F}^{\prime \prime}$ must be stable, hence it is the structure sheaf of a conic, hence it is generated by one global section. Thus the map $\mathscr{F}_{F} \rightarrow \mathscr{F}^{\prime \prime}$ is surjective on global sections, forcing $h^{0}\left(\mathscr{F}^{\prime}\right)=2$. Thus $h^{1}\left(\mathscr{F}^{\prime}\right)=0$ which, together with $h^{1}\left(\mathscr{F}^{\prime \prime}\right)=0$, implies that $h^{1}(\mathscr{F})=0$. Contradiction.

Remark 5.5. Let $\varphi$ be a $4 \times 3$-matrix with entries in $V^{*}$ which is semistable: Modulo operations on rows and columns, $\varphi$ is not equivalent to a matrix having a zero row, a zero $2 \times 2$-submatrix, or a zero $3 \times 1$-submatrix. Then one of the maximal minors of $\varphi$ is not zero.

Proof. Assume that all maximal minors of $\varphi$ are zero. Each $3 \times 3$-submatrix $\psi$ of $\varphi$ satisfies the hypotheses of remark Remark 5.6, hence it is equivalent to $\psi_{1}$ or $\psi_{2}$. We can choose $\psi$ to have a zero entry, thus ruling out $\psi_{2}$. From the assumption that all minors of $\varphi$ are zero it is easy to deduce that the row of $\varphi$ which is not part of $\psi$ is a linear combination of the rows of $\psi$; see the proof of Claim 6.6. This contradicts the semistability of $\varphi$.

Remark 5.6. Let $\psi$ be a $3 \times 3$-matrix with entries in $V^{*}$ and zero determinant. Assume that $\psi$ is equivalent to neither of the following matrices:

$$
\left[\begin{array}{lll}
0 & \star & \star \\
0 & \star & \star \\
0 & \star & \star
\end{array}\right] \text { or }\left[\begin{array}{lll}
0 & 0 & \star \\
0 & 0 & \star \\
\star & \star & \star
\end{array}\right] \text { or }\left[\begin{array}{lll}
0 & 0 & 0 \\
\star & \star & \star \\
\star & \star & \star
\end{array}\right]
$$

Then $\psi$ is equivalent to one of the following matrices:

$$
\psi_{1}=\left[\begin{array}{ccc}
X & Y & 0 \\
Z & 0 & Y \\
0 & -Z & X
\end{array}\right] \quad \text { or } \psi_{2}=\left[\begin{array}{lll}
X & Y & Z \\
Y & a_{1} X+a_{2} Y & a_{3} X+a_{4} Y+a_{5} Z \\
Z & a_{6} X+a_{7} Y+a_{8} Z & a_{9} X+a_{10} Z
\end{array}\right]
$$


with $a_{1}, \ldots, a_{10} \in k^{*}$. Modulo operations on rows and columns $\psi_{2}$ is not equivalent to a matrix having a zero entry.

Proof. We distinguish two cases: either $\psi$ has one zero entry or, modulo equivalence, all entries of $\psi$ are nonzero. In the second case $\psi \sim \psi_{2}$. In the first case we may assume that $\psi_{11}=X, \psi_{12}=Y, \psi_{13}=0$. We now consider two subcases: $\operatorname{span}\left\{\psi_{23}, \psi_{33}\right\}$ is equal to or is different from $\operatorname{span}\{X, Y\}$. In the second subcase we may write

$$
\psi=\left[\begin{array}{ccc}
X & Y & 0 \\
\psi_{21} & \psi_{22} & Y \\
\psi_{31} & \psi_{32} & Z
\end{array}\right]
$$

We have

$$
\operatorname{det}(\psi)=X Z \psi_{22}+Y^{2} \psi_{31}-X Y \psi_{32}-Y Z \psi_{21}=0,
$$

forcing $\psi_{22}=a Y$. Performing operations on rows we may assume that $\psi_{22}=0$. Thus $Y \psi_{31}-X \psi_{32}-Z \psi_{21}=0$. We get

$$
\psi_{31}=c Z \text { modulo } X, \quad \psi_{21}=c Y \text { modulo } X \text {. }
$$

But then

$$
\psi \sim\left[\begin{array}{ccc}
X & Y & 0 \\
0 & \star & Y \\
0 & \star & Z
\end{array}\right]
$$

From $\operatorname{det}(\psi)=0$ we get

$$
\psi \sim\left[\begin{array}{ccc}
X & Y & 0 \\
0 & 0 & Y \\
0 & 0 & Z
\end{array}\right],
$$

contradiction. This eliminates the second subcase.

Finally, we may assume that

$$
\psi=\left[\begin{array}{ccc}
X & Y & 0 \\
\psi_{21} & \psi_{22} & Y \\
\psi_{31} & \psi_{32} & X
\end{array}\right] .
$$

We have

$$
\operatorname{det}(\psi)=X^{2} \psi_{22}+Y^{2} \psi_{31}-X Y \psi_{32}-X Y \psi_{21}=0
$$

hence

$$
\psi_{22}=a Y, \quad \psi_{31}=b X, \quad \psi_{21}+\psi_{32}=a X+b Y .
$$

Performing operations on rows we may assume that $a=b=0$. Denoting $Z=\psi_{21}$ we arrive at $\psi \sim \psi_{1}$. 


\section{Sheaves $\mathscr{F}$ with $h^{0}(\mathscr{F}(-1)) \neq 0$ and $h^{1}(\mathscr{F})=0$}

Let $\mathscr{F}$ be a sheaf on $\mathbb{P}^{2}$ with $h^{0}(\mathscr{F}(-1))=p \neq 0, h^{1}(\mathscr{F})=0$ and Hilbert polynomial $P_{\mathscr{F}}(t)=a t+b, 0 \leq b<a$. From the Beilinson complex we deduce that $\mathscr{F}$ has to have one of the following resolutions:

(i) when $a<2 b$

$0 \rightarrow p \mathscr{O}(-2) \stackrel{\psi}{\rightarrow}(p+a-b) \mathcal{O}(-2) \oplus(m+2 b-a) \mathcal{O}(-1) \stackrel{\varphi}{\rightarrow} m \mathscr{O}(-1) \oplus b \mathscr{O} \rightarrow \mathscr{F} \rightarrow 0$,

(ii) when $a \geq 2 b$

$0 \rightarrow p \mathscr{O}(-2) \stackrel{\psi}{\rightarrow}(p+a-b) \mathcal{O}(-2) \oplus m \mathcal{O}(-1) \stackrel{\varphi}{\rightarrow}(m+a-2 b) \mathscr{O}(-1) \oplus b \mathcal{O} \rightarrow \mathscr{F} \rightarrow 0$,

where $m$ is an integer, $\psi_{11}=0$ and $\varphi_{12}=0$. In case (ii) we must have $m \geq 2$ because, if $m=1$, then we get the contradiction $\psi=0$. We obtain the following exact commutative diagram, say in case (ii):

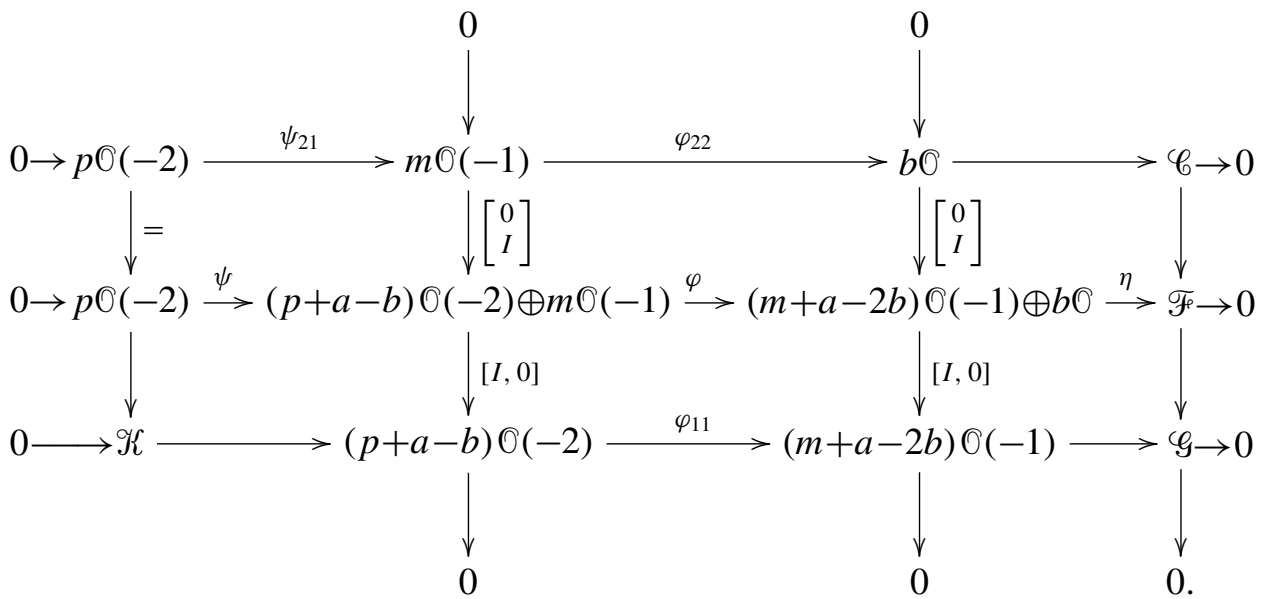

This induces the exact sequence

$$
0 \rightarrow \mathscr{K} \rightarrow \mathscr{b} \rightarrow \mathscr{F} \rightarrow \mathscr{G} \rightarrow 0
$$

Note that $\mathscr{K}$ is torsion-free or zero, because it is a subsheaf of the torsion-free sheaf $(p+a-b) \mathcal{O}(-2)$.

Remark 6.1. Assume that $\mathscr{F}$ is semistable. Then $\mathscr{b}$ does not have zero-dimensional torsion and is not supported on a curve.

Proof. Let $\mathscr{T}$ be the zero-dimensional torsion of $\mathscr{C}$. As $\mathscr{F}$ has no zero-dimensional torsion it follows that the induced map $\mathscr{T} \rightarrow \mathscr{F}$ is zero. Thus $\mathscr{T}$ is a subheaf of $\mathscr{K}$. The latter is torsion-free, so $\mathscr{T}=0$. 
Assume now that $\mathscr{C}$ is supported on a curve. Then $m=p+b$ and $\mathscr{K}=0$. Thus $\mathscr{C}$ is a subsheaf of $\mathscr{F}$. We have

$$
P_{\mathscr{C}}(t)=b\left(\begin{array}{c}
t+2 \\
2
\end{array}\right)-(p+b)\left(\begin{array}{c}
t+1 \\
2
\end{array}\right)+p\left(\begin{array}{l}
t \\
2
\end{array}\right)=(b-p) t+b .
$$

But $b /(b-p)>b / a$, which shows that $\mathscr{b}$ violates the semistability of $\mathscr{F}$.

Remark 6.2. Assume that $\mathscr{F}$ is semistable. Then, in case (i), we either have $m+2 b-a<b$ or $m+2 b-a \geq b$ and all maximal minors of $\varphi_{22}$ are zero. Similarly, in case (ii), either $m<b$ or $m \geq b$ and all maximal minors of $\varphi_{22}$ are zero. This follows from Remark 6.1.

Remark 6.3. $\eta$ is an isomorphism on global sections. As a consequence, if $\mathscr{F}$ is semistable, $\varphi_{22}$ cannot have the form

$$
\left[\begin{array}{ccccc}
0 & 0 & \star & \cdots & \star \\
\vdots & \vdots & \vdots & & \vdots \\
0 & 0 & \star & \cdots & \star \\
X & Y & \star & \cdots & \star
\end{array}\right] .
$$

Indeed, if $\varphi_{22}$ had the above form, we would get the commutative diagram

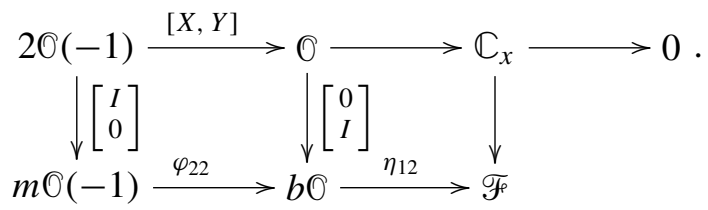

Here $\mathbb{C}_{x}$ is the structure sheaf of the point $x=(0: 0: 1)$. But the map $\mathbb{C}_{x} \rightarrow \mathscr{F}$ is zero because $\mathscr{F}_{F}$ does not have zero-dimensional torsion. This shows that $\eta_{12}$ has nontrivial kernel. This contradicts the fact that $\eta$ is an isomorphism on global sections.

Claim 6.4. There are no semistable sheaves $\mathscr{F}$ on $\mathbb{P}^{2}$ with $h^{0}(\mathscr{F}(-1)) \neq 0, h^{1}(\mathscr{F})=$ 0 and Hilbert polynomial

$$
P_{\mathscr{F}}(t)=n t+1, \quad n \geq 2 \quad \text { or } \quad P_{\mathscr{F}}(t)=n t+2, \quad n \geq 3 .
$$

Proof. The case $P_{\mathscr{F}}(t)=n t+1$ follows directly from Remark 6.3 because $\varphi_{22}$ must have the form

$$
[X, Y, \star, \cdots, \star] .
$$

In the case $P_{\mathscr{F}}(t)=n t+2$ all $2 \times 2$-minors of $\varphi_{22}$ are zero, cf. Remark 6.2. It follows that $\varphi_{22}$ has the form

$$
\left[\begin{array}{lllll}
0 & 0 & \star & \star & \star \\
X & Y & \star & \cdots & \star
\end{array}\right] .
$$


The claim follows from Remark 6.3.

Remark 6.5. Let $\alpha=\left(\alpha_{i j}\right)$ be a morphism of sheaves on $\mathbb{P}^{n}=\mathbb{P}(V)$ :

$$
\alpha:(m+1) \mathcal{O} \rightarrow m \mathscr{O}(l) .
$$

Assume that at least one of the maximal minors of $\alpha$ is a nonzero polynomial. Then Fer $(\alpha) \simeq \mathcal{O}(-d)$ where $d$ is an integer satisfying $0 \leq d \leq m l$. More precisely, let $\alpha_{i}, 1 \leq i \leq m+1$, denote the minor obtained from $\alpha$ by erasing the $i^{\text {th }}$ column. Let

$$
\beta=\left(\beta_{1}, \ldots, \beta_{m+1}\right), \quad \text { where } \quad \beta_{i}=\frac{(-1)^{i} \alpha_{i}}{\text { g.c.d. }\left(\alpha_{1}, \ldots, \alpha_{m+1}\right)}, \quad 1 \leq i \leq m+1 .
$$

Let $d$ be the degree of the entries of $\beta$. Then we have the exact sequence

$$
0 \rightarrow \mathcal{O}(-d) \stackrel{\beta}{\rightarrow}(m+1) \mathcal{O} \stackrel{\alpha}{\rightarrow} m \mathcal{O}(l) .
$$

Claim 6.6. Let $\mathscr{F}_{\mathrm{F}}$ be a semistable sheaf on $\mathbb{P}^{2}=\mathbb{P}(V)$ with $h^{0}(\mathscr{F}(-1)) \neq 0$, $h^{1}(\mathscr{F})=0$ and Hilbert polynomial $P_{\mathscr{F}}(t)=n t+3, n \geq 4$. Then $h^{0}(\mathscr{F}(-1))=1$ and $\mathscr{F}$ has a resolution

$$
0 \rightarrow \mathcal{O}(-2) \stackrel{\psi}{\rightarrow}(n-2) \mathcal{O}(-2) \oplus 3 \mathcal{O}(-1) \stackrel{\varphi}{\rightarrow}(n-3) \mathcal{O}(-1) \oplus 3 \mathcal{O} \rightarrow \mathscr{F} \rightarrow 0
$$

with $\varphi_{12}=0, \psi_{11}=0, \varphi_{21} \neq 0$,

$$
\psi_{21} \sim\left[\begin{array}{c}
X \\
Y \\
Z
\end{array}\right], \quad \varphi_{22} \sim\left[\begin{array}{rrr}
-Y & X & 0 \\
-Z & 0 & X \\
0 & -Z & Y
\end{array}\right], \quad \varphi_{11} \nsim\left[\begin{array}{cc}
\varphi^{\prime} & 0 \\
\star & \star
\end{array}\right]
$$

where $\varphi^{\prime}$ is an $m \times m$-matrix with entries in $V^{*}, 1 \leq m \leq n-3$. Moreover, F is an extension of the form

$$
0 \rightarrow \mathrm{O}_{C}(1) \rightarrow \mathscr{F} \rightarrow \mathscr{G} \rightarrow 0
$$

where $C$ is a curve of degree $d, 4 \leq d \leq n$, and the map $\mathscr{F} \rightarrow \mathscr{G}$ is zero on global sections. If $n \geq 7$ then $d \geq 5$.

Proof. Assume $n \geq 6$ so that we are in case (ii). If $m \geq 5$ then $\varphi_{22}$ has the form

$$
\left[\begin{array}{llll}
\star \star \star & 0 & \cdots & 0 \\
\star \star \star & \cdots & \star \\
\star \star \star & \star & \cdots & \star
\end{array}\right]=\left[\begin{array}{l}
\star \star \star \\
\star \star \star \varphi^{\prime} \\
\star \star \star
\end{array}\right] \text {. }
$$

From Remark 6.2 we see that all $2 \times 2$-minors of $\varphi^{\prime}$ are zero. Since $\varphi_{22}$ cannot have a zero column it follows that

$$
\varphi_{22} \sim\left[\begin{array}{lllll}
\star \star & \star & 0 & \cdots & 0 \\
\star \star \star & 0 & \cdots & 0 \\
\star \star \star & \star & \cdots & \star
\end{array}\right]
$$


By virtue of Remark 6.3 this is impossible.

Assume now that $m=4$. Firstly, we notice that $\varphi_{22}$ cannot have a zero row because, if

$$
\varphi_{22}=\left[\begin{array}{ccc}
0 & \cdots & 0 \\
& \varphi^{\prime} &
\end{array}\right]
$$

then, arguing as in Remark 6.1, we get that $\varphi^{\prime}$ has all maximal minors equal to zero hence $\varphi^{\prime}$ has one row identically zero. This, again, contradicts Remark 6.3. Secondly, using the same kind of arguments, we notice that $\varphi_{22}$ cannot have the form

$$
\left[\begin{array}{llll}
X & 0 & 0 & 0 \\
\star & \star & \star & \star \\
\star & \star & \star & \star
\end{array}\right]
$$

Now $\varphi_{22}$ has nontrivial kernel in $\oplus_{4} V^{*}$ by hypothesis. No element in the kernel can have the form

$$
\left[\begin{array}{l}
X \\
Y \\
0 \\
0
\end{array}\right]
$$

otherwise we would arrive at a matrix excluded by Remark 6.3:

$$
\varphi_{22} \sim\left[\begin{array}{rrrr}
0 & 0 & \star & \star \\
0 & 0 & \star & \star \\
-Y & X & \star & \star
\end{array}\right] .
$$

Performing operations on the columns of $\varphi_{22}$ we may assume that

$$
\left[\begin{array}{l}
X \\
Y \\
Z \\
0
\end{array}\right]
$$

is in the kernel of $\varphi_{22}$. Performing operations on the rows of $\varphi_{22}$ we may assume that

$$
\varphi_{22}=\left[\begin{array}{rrrr}
-Y & X & 0 & u \\
-Z & 0 & X & v \\
0 & -Z & Y & w
\end{array}\right]
$$

From

$$
0=\left|\begin{array}{rrc}
-Y & X & u \\
-Z & 0 & v \\
0 & -Z & w
\end{array}\right|=Z^{2} u-Y Z v+X Z w
$$


we get $Z u-Y v+X w=0$, which shows that the fourth column of $\varphi_{22}$ is a linear combination of the first three columns. Thus $\varphi$ is equivalent to a matrix having a zero column, contradiction.

The case $m=2$ is excluded by using Remark 6.3. We conclude that $m=3$ and, from what was said above, that we have

$$
\psi_{21} \sim\left[\begin{array}{c}
X \\
Y \\
Z
\end{array}\right], \quad \varphi_{22} \sim\left[\begin{array}{rrr}
-Y & X & 0 \\
-Z & 0 & X \\
0 & -Z & Y
\end{array}\right] .
$$

Thus far we have obtained the desired resolution of $\mathscr{F}$ in the cases $n \geq 6$. The cases $n=4$ and $n=5$ are completely analogous. From our concrete description of $\varphi_{22}$ we see that $\mathscr{C} \simeq \mathcal{O}(1)$. Since $\mathscr{F}$ surjects onto $\mathscr{G}$, the latter has support of dimension zero or one. Thus, at least one of the maximal minors of $\varphi_{11}$ must be a nonzero polynomial. We can apply Remark 6.5 to conclude that $\mathscr{H e r}\left(\varphi_{11}\right) \simeq$ $\mathcal{O}(-d+1)$ for some integer $d \geq 3$. We have

$$
P \mathscr{G}(t)=(n-3)\left(\begin{array}{c}
t+1 \\
2
\end{array}\right)-(n-2)\left(\begin{array}{l}
t \\
2
\end{array}\right)+\left(\begin{array}{c}
t+3-d \\
2
\end{array}\right)=(n-d) t+\frac{(d-2)(d-3)}{2} .
$$

The sheaf $\mathscr{G}$ violates the semistability of $\mathscr{F}$ precisely when

$$
\frac{(d-2)(d-3)}{2(n-d)}<\frac{3}{n}, \quad \text { that is, } \quad n(d-5)<-6 .
$$

Thus, we cannot have $d=3$ and, if $d=4$, then $n \leq 6$. We conclude that $\mathscr{F}$ is an extension

$$
0 \rightarrow \mathrm{O}_{C}(1) \rightarrow \mathscr{F}_{F} \rightarrow \mathscr{G} \rightarrow 0
$$

with $\operatorname{deg}(C)=d \geq 4$, respectively $\operatorname{deg}(C) \geq 5$ in the case $n \geq 7$. Finally, we cannot have

$$
\varphi_{11} \sim\left[\begin{array}{cc}
\varphi^{\prime} & 0 \\
\star & \star
\end{array}\right] \quad \text { with } \quad \varphi^{\prime}: m \mathscr{O}(-2) \rightarrow m \mathscr{O}(-1), \quad 1 \leq m \leq n-3 .
$$

Indeed, if this were the case, then, since $\varphi_{11}$ has at least one nonzero maximal minor, we would get $\operatorname{det}\left(\varphi^{\prime}\right) \neq 0$ and a surjection $\mathscr{F} \rightarrow \operatorname{Coker}\left(\varphi^{\prime}\right)$ onto a sheaf with Hilbert polynomial $P(t)=m t$. This would contradict the semistability of $\mathscr{F}$.

Lemma 6.7. Let $C \subset \mathbb{P}^{2}$ be a curve given by the equation $f=0$, where $f(X, Y, Z)$ is a homogeneos polynomial. Let $\mathscr{I} \subset \mathrm{O}_{C}$ be a sheaf of ideals. Then there is a homogeneous polynomial $g(X, Y, Z)$ dividing $f$ such that the sheaf of ideals $\mathscr{F} \subset \mathrm{O}_{C}$ generated by $g$ satisfies: $\mathscr{I} \subset \mathscr{F}$ and $\mathscr{F} / \mathscr{I}$ is supported on finitely many points. 
Proof. Dehomogenizing in a suitable open affine subset we reduce the problem to the following: let $f(X, Y)$ be a polynomial in $k[X, Y]$. Let $I \subset k[X, Y]$ be an ideal containing $f$. Then there is a polynomial $g(X, Y)$ dividing $f$ such that $I \subset\langle g\rangle$ and $\langle g\rangle / I$ is supported on finitely many points.

Let $f=f_{1}^{n_{1}} \ldots f_{\kappa}^{n_{\kappa}}$ be the decomposition of $f$ into irreducible factors. Let

$$
I=\mathfrak{q}_{1} \cap \ldots \cap \mathfrak{q}_{m} \cap \mathfrak{a}_{1} \cap \ldots \cap \mathfrak{a}_{l}
$$

be a primary decomposition of $I$. Here $m \leq \kappa, \mathfrak{q}_{i}$ is a primary ideal associated to $\left\langle f_{i}\right\rangle$ and $\mathfrak{a}_{1}, \ldots, \mathfrak{a}_{l}$ are primary ideals associated to maximal ideals $\mathfrak{m}_{1}, \ldots, \mathfrak{m}_{l}$. Put

$$
\mathfrak{q}=\mathfrak{q}_{1} \cap \ldots \cap \mathfrak{q}_{m} .
$$

We notice that $\mathfrak{q} / I$ is supported on $\mathfrak{m}_{1}, \ldots, \mathfrak{m}_{l}$. For $1 \leq i \leq m$ let $r_{i}$ be the largest integer such that $\mathfrak{q}_{i} \subset\left\langle f_{i}^{r_{i}}\right\rangle$. We claim that $g=f_{1}^{r_{1}} \cdot \ldots \cdot f_{m}^{r_{m}}$ is the desired polynomial. To prove this it is enough to show that $\langle g\rangle / \mathfrak{q}$ is supported on finitely many points. Since localization commutes with intersections it is enough to show that each $\left\langle f_{i}^{r_{i}}\right\rangle / \mathfrak{q}_{i}$ is supported on finitely many points.

So far we have reduced the problem to the following: let $f \in k[X, Y]$ be an irreducible polynomial. Let $\mathfrak{q} \subset k[X, Y]$ be a primary ideal associated to $\langle f\rangle$. Let $r \geq 1$ be the largest integer such that $\mathfrak{q} \subset\left\langle f^{r}\right\rangle$. Then $\left\langle f^{r}\right\rangle / \mathfrak{q}$ is supported on finitely many points.

We may assume that $\mathfrak{q}$ is not a power of $\langle f\rangle$. Let $s$ be the smallest integer such that $\left\langle f^{s}\right\rangle \subset \mathfrak{q}$. We will prove the above statement by induction on $s$. If $s=r+1$ then

$$
\left\langle f^{r}\right\rangle / \mathfrak{q} \simeq \frac{\left\langle f^{r}\right\rangle /\left\langle f^{r+1}\right\rangle}{\mathfrak{q} /\left\langle f^{r+1}\right\rangle}
$$

can be regarded as the structure sheaf of a proper subscheme of the scheme $X \subset \mathbb{P}^{2}$ given by $\{f=0\}$. This is so because

$$
\left\langle f^{r}\right\rangle /\left\langle f^{r+1}\right\rangle \simeq k[X, Y] /\langle f\rangle \quad \text { as } \quad k[X, Y] \text { - modules. }
$$

But $X$ is an irreducible scheme of dimension one, hence any proper subscheme has dimension zero.

Assume now that $s>r+1$ and the statement is true for any ideal $\mathfrak{q}^{\prime}$ satisfying $\mathfrak{q}^{\prime} \subset\left\langle f^{r}\right\rangle, \quad \mathfrak{q}^{\prime} \nsubseteq\left\langle f^{r+1}\right\rangle, \quad\left\langle f^{s-1}\right\rangle \varsubsetneqq \mathfrak{q}^{\prime}$. Such an ideal is $\mathfrak{q}^{\prime}=\mathfrak{q}+\left\langle f^{s-1}\right\rangle$. By the induction hypothesis we know that $\left\langle f^{r}\right\rangle / \mathfrak{q}^{\prime}$ is supported on finitely many points. To finish the proof it is enough to show that $\mathfrak{q}^{\prime} / \mathfrak{q}$ is supported on finitely many points. But

$$
\mathfrak{q}^{\prime} / \mathfrak{q} \simeq\left\langle f^{s-1}\right\rangle / \mathfrak{q} \cap\left\langle f^{s-1}\right\rangle
$$


If $\mathfrak{q} \cap\left\langle f^{s-1}\right\rangle \neq\left\langle f^{s}\right\rangle$ then the right-hand side is supported on finitely many points by the first step in the induction argument. Now choose $h \in \mathfrak{q} \backslash\left\langle f^{r+1}\right\rangle$. Then $f^{s-r-1} h \in \mathfrak{q} \cap\left\langle f^{s-1}\right\rangle \backslash\left\langle f^{s}\right\rangle$. This finishes the proof of the lemma.

In the remaining part of this section we will seek more precise information about the morphisms occuring in Claim 6.6. For a start, assume that $\mathscr{F}_{F}$ is an arbitrary sheaf having a resolution as in Claim 6.6; we determine which subsheaves $\mathscr{F}^{\prime} \subset \mathscr{F}^{\prime}$ are destabilizing. Let $\mathscr{G}^{\prime}$ be the image of $\mathscr{F}^{\prime}$ in $\mathscr{G}$ and let $\mathscr{I}(1)$ be the preimage of $\mathscr{F}^{\prime}$ in $\mathscr{O}_{C}(1)$. Here $\mathscr{I}$ is the ideal sheaf of a subscheme of $C$. By Lemma 6.7 we can find a curve $C^{\prime} \subset C$ such that the ideal sheaf $\mathscr{g}$ of $C^{\prime}$ contains $\mathscr{I}$ and $P_{\mathscr{I}(1)}(t)=P_{\mathscr{f}(1)}(t)-c$, where $c$ is a nonnegative integer. From the exact sequence

$$
0 \rightarrow \mathscr{I}(1) \rightarrow \mathscr{F}^{\prime} \rightarrow \varphi^{\prime} \rightarrow 0
$$

we get

$$
P_{\mathscr{F}^{\prime}}(t)=P_{\mathscr{I}(1)}(t)+P_{\mathscr{G}^{\prime}}(t)=P_{\mathscr{F}(1)}(t)+P_{\mathscr{G}^{\prime}}(t)-c .
$$

Put $\kappa=\operatorname{deg}\left(C^{\prime}\right)$. We allow $\kappa=0$ for the case $\mathscr{g}=0_{C}$. From the exact sequence

$$
0 \rightarrow \mathrm{O}(-d+1) \rightarrow \mathrm{O}(-\kappa+1) \rightarrow \mathscr{g}(1) \rightarrow 0
$$

we see that $h^{0}(\mathscr{F}(1))=0$ if $\kappa \geq 2$. But then $h^{0}(\mathscr{I}(1))=0$, forcing the map $H^{0}\left(\mathscr{F}^{\prime}\right) \rightarrow H^{0}\left(\mathscr{G}^{\prime}\right)$ to be injective. Since the map $\mathscr{F}^{\rightarrow} \mathscr{G}$ is zero on global sections we see that $h^{0}\left(\mathscr{F}^{\prime}\right)=0$. It follows that $\mathscr{F}^{\prime}$ does not violate the semistability of $\mathscr{F}^{\prime}$.

In the case $\kappa=0$ we have $P_{\mathscr{F} / \mathscr{F}^{\prime}}(t)=c+P_{\mathscr{G} / \mathscr{G}^{\prime}}(t)$, hence $\mathscr{F}^{\prime}$ violates the semistability of $\mathscr{F}$ if and only if $\alpha_{1}\left(\mathscr{G} / \mathscr{G}^{\prime}\right)>0$ and

$$
\frac{\alpha_{0}\left(\mathscr{G} / \mathscr{G}^{\prime}\right)+c}{\alpha_{1}\left(\mathscr{G} / \mathscr{G}^{\prime}\right)}<\frac{3}{n}
$$

Assume now that $\kappa=1$. We have

$$
P_{\mathscr{F}^{\prime} / \mathscr{F}^{\prime}}(t)=P_{\mathscr{O}_{C}(1) / \mathscr{F}(1)}(t)+P_{\mathscr{G}_{/} / \mathscr{G}^{\prime}}(t)=t+2+c+P_{\mathscr{G} / \mathscr{G}^{\prime}},
$$

hence $\mathscr{F}^{\prime}$ violates semistability if and only if

$$
\frac{2+c+\alpha_{0}\left(\mathscr{G} / \mathscr{G}^{\prime}\right)}{1+\alpha_{1}\left(\mathscr{G} / \mathscr{G}^{\prime}\right)}<\frac{3}{n}
$$

Now the exact sequence

$$
0 \rightarrow \mathrm{O}_{C}(1) \rightarrow \mathscr{F} \rightarrow \mathscr{G} \rightarrow 0
$$

together with the hypothesis $h^{1}\left(\mathscr{F}_{F}(i)\right)=0$ for $i \geq 0$ give $h^{1}(\mathscr{G}(i))=0$ for $i \geq 0$. This and the exact sequence

$$
0 \rightarrow \mathscr{G}^{\prime} \rightarrow \mathscr{G} \rightarrow \mathscr{G} / \mathscr{G}^{\prime} \rightarrow 0
$$


yield $h^{1}\left(\mathscr{G} / \mathscr{G}^{\prime}(i)\right)=0$ for $i \geq 0$. In particular $\alpha_{0}\left(\mathscr{G} / \mathscr{G}^{\prime}\right)=h^{0}\left(\mathscr{G} / \mathscr{G}^{\prime}\right) \geq 0$. This eliminates the case $\alpha_{1}\left(\mathscr{G} / \mathscr{G}^{\prime}\right)=0$ from above. We summarize our findings so far:

Remark 6.8. $\mathscr{F}$ is semistable if and only if there are no quotients sheaves $\mathscr{E}$ of $\mathscr{G}$ satisfying

$$
h^{1}(\mathscr{E})=h^{1}(\mathscr{E}(1))=0 \quad \text { and } \quad 0 \leq \alpha_{0}(\mathscr{E})<\frac{3}{n} \alpha_{1}(\mathscr{E}) \neq 0 .
$$

One direction was proved in the discussion above. The other direction follows by taking $\kappa=0$ and $c=0$, in other words taking $\mathscr{F}^{\prime}$ to be the preimage of $\mathscr{G}^{\prime}$, where $\mathscr{G}^{\prime}$ is the kernel of the surjection $\mathscr{G} \rightarrow \mathscr{E}$.

Claim 6.9. Let $\mathscr{F}$ be a sheaf on $\mathbb{P}^{2}$ with resolution

$$
0 \rightarrow \mathcal{O}(-2) \rightarrow(n-2) \mathcal{O}(-2) \oplus 3 \mathcal{O}(-1) \rightarrow(n-3) \mathcal{O}(-1) \oplus 3 \mathfrak{O} \rightarrow \mathscr{F} \rightarrow 0
$$

satisfying the properties from Claim 6.6. Assume that $n \in\{4,5,6,7\}$. Then $\mathscr{F}$ is semistable.

Proof. Assume that there is $\mathscr{E}$ as in Remark 6.8. We must have $\alpha_{0}(\mathscr{E})=0$, otherwise

$$
1<\frac{3}{n} \alpha_{1}(\mathscr{E}) \leq \frac{3}{n} \alpha_{1}(\mathscr{G})=\frac{3}{n}(n-d), \quad \text { forcing } \quad \frac{3 d}{2}<n .
$$

This gives $n \geq 8$, contradicting our hypothesis.

The Beilinson sequence of $\mathscr{E}(1)$ leads to the following resolution:

$$
0 \rightarrow m \mathscr{O}(-2) \rightarrow m \mathscr{O}(-1) \rightarrow \mathscr{E} \rightarrow 0
$$

for some integer $m \leq n-d$. In the case $n=4$ there is no such $\mathscr{E}$. In the case $n=5$ we have $m=1$. In the cases $n \in\{6,7\}$ we have $m \in\{1,2\}$. We obtain a commutative exact diagram

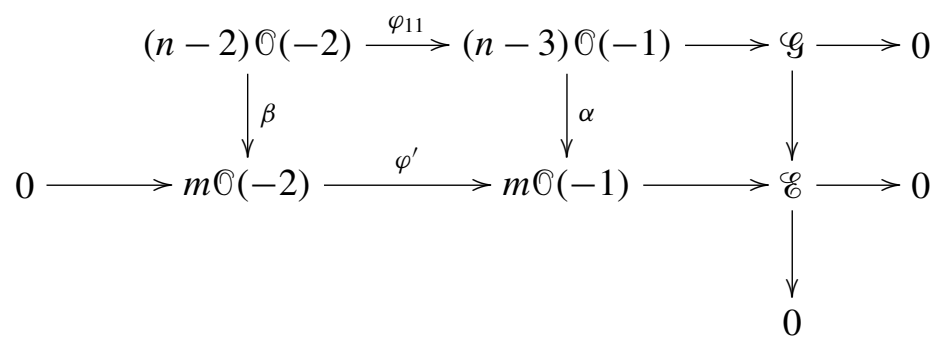

with $\alpha \neq 0$ because the following composition is surjective:

$$
(n-3) \mathcal{O}(-1) \stackrel{\alpha}{\rightarrow} m \mathscr{O}(-1) \rightarrow \stackrel{\mathscr{E}}{ } .
$$

After performing operations on the rows and columns of $\varphi_{11}$ and $\varphi^{\prime}$ it is always possible to write

$$
\alpha=\left[\begin{array}{cc}
I_{r} & 0 \\
0 & 0
\end{array}\right], \quad \beta=\left[\begin{array}{cc}
I_{s} & 0 \\
0 & 0
\end{array}\right]
$$


We arrive at

$$
\varphi_{11} \sim\left[\begin{array}{cc}
\varphi^{\prime \prime} & 0 \\
\star & \star
\end{array}\right]
$$

with $\varphi^{\prime \prime}$ an $r \times s$-matrix. But $r \geq \operatorname{rank}\left(\alpha \varphi_{11}\right)=\operatorname{rank}\left(\varphi^{\prime} \beta\right)=s$. This contradicts the assumption on $\varphi_{11}$ and finishes the proof of the claim.

Claim 6.10. Let $\mathscr{F}$ be a sheaf on $\mathbb{P}^{2}$ with $P_{\mathscr{F}}(t)=n t+3, h^{1}(\mathscr{F})=0, h^{0}(\mathscr{F}(-1)) \neq 0$. Assume that $8 \leq n \leq 15$. Then $\mathscr{F}$ is semistable if and only if it has a resolution

$$
0 \rightarrow \mathcal{O}(-2) \rightarrow(n-2) \mathcal{O}(-2) \oplus 3 \mathcal{O}(-1) \stackrel{\varphi}{\rightarrow}(n-3) \mathcal{O}(-1) \oplus 3 \mathcal{O} \rightarrow \mathscr{F} \rightarrow 0
$$

satisfying the properties from Claim 6.6 and, in addition, the following property: $\varphi_{11}$ is not equivalent to a matrix of the form

$$
\left[\begin{array}{ll}
\varphi^{\prime} & 0 \\
\star & \star
\end{array}\right] \quad \text { where } \quad \varphi^{\prime}:(m+1) \mathcal{O}(-2) \rightarrow m \mathscr{O}(-1)
$$

is a morphism having kernel $O(-3)$ and $m$ is an integer satisfying $m>\frac{n}{3}+1$.

Proof. One direction is clear: if $\mathscr{F}$ is semistable then it has a resolution as in Claim 6.6. If $\varphi$ did not satisfy the "additional property" then $\mathscr{F}$ would surject onto a sheaf $\mathscr{E}$ with resolution

$$
0 \rightarrow \mathscr{O}(-3) \rightarrow(m+1) \mathscr{O}(-2) \rightarrow m \mathscr{O}(-1) \rightarrow \mathscr{E} \rightarrow 0 .
$$

But

$$
P_{\mathscr{E}}(t)=m\left(\begin{array}{c}
t+1 \\
2
\end{array}\right)-(m+1)\left(\begin{array}{l}
t \\
2
\end{array}\right)+\left(\begin{array}{c}
t-1 \\
2
\end{array}\right)=(m-1) t+1,
$$

which shows that $\mathscr{E}$ violates the semistability of $\mathscr{F}$ precisely when

$$
\frac{1}{m-1}<\frac{3}{n}, \quad \text { that is, } \frac{n}{3}+1<m \text {. }
$$

Conversely, we assume that $\mathscr{F}$ has the resolution from the claim and let $\mathscr{E}$ be a sheaf as in Remark 6.8. Our aim is to arrive at a contradiction. Since $n \leq 15$ we must have $\alpha_{0}(\mathscr{E})=0$ or $\alpha_{0}(\mathscr{E})=1$. In the first case the argument is the same as in Claim 6.9. Now assume that $\alpha_{0}(\mathscr{E})=1$ and write $P_{\mathscr{E}}(t)=m t+1$. We have

$$
h^{0}(\mathscr{E})=1, \quad h^{1}(\mathscr{E})=0, \quad h^{0}(\mathscr{E}(1))=m+1, \quad h^{1}(\mathscr{E}(1))=0
$$

so the Beilinson sequence (4.1) of $\mathscr{E}(1)$ gives the resolution

$$
0 \rightarrow \mathfrak{O}(-2) \rightarrow(p+m+2) \mathscr{O}(-1) \stackrel{\rho}{\rightarrow} p \mathscr{O}(-1) \oplus(m+1) \mathscr{O} \rightarrow \mathscr{E}(1) \rightarrow 0 .
$$

Here $p$ is some integer and from the fact that $\mathscr{E}$ is supported on a curve we get $\operatorname{rank}\left(\rho_{11}\right)=p=0$. So far we have obtained a resolution

$$
0 \rightarrow \mathcal{O}(-3) \rightarrow(m+2) \mathcal{O}(-2) \stackrel{\varphi^{\prime}}{\rightarrow}(m+1) \mathcal{O}(-1) \rightarrow \mathscr{E} \rightarrow 0
$$


that fits into an exact commutative diagram

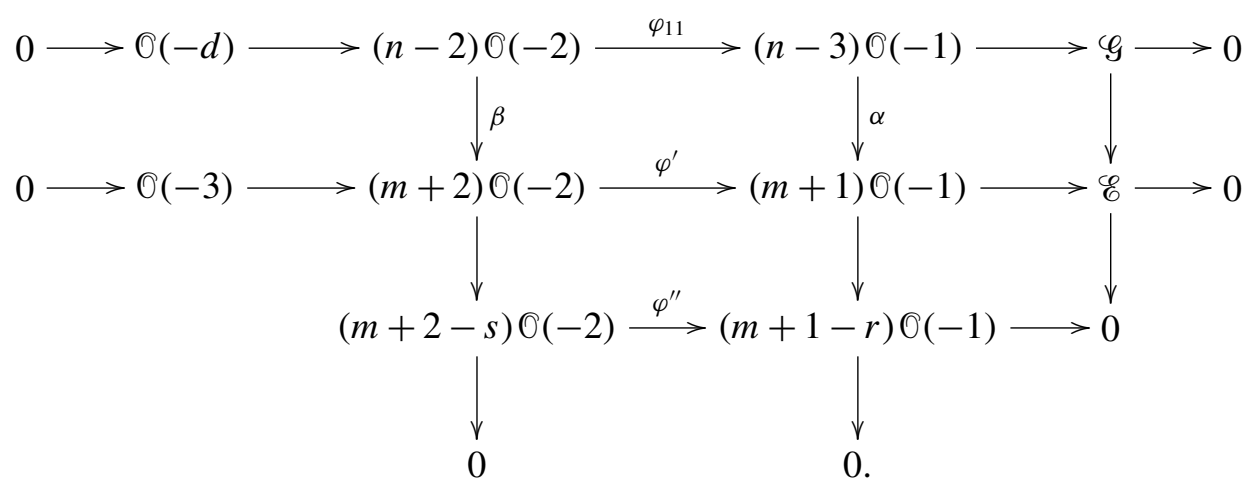

Here $r, s$ are the ranks of $\alpha, \beta$. Since $\varphi^{\prime \prime}$ is surjective we must have either $m+2-$ $s>m+1-r>0$, i.e., $m+1>r>s-1$, or $r=m+1$. In the first case

$$
\varphi_{11} \sim\left[\begin{array}{cc}
\psi & 0 \\
\star & \star
\end{array}\right]
$$

with $\psi$ an $r \times s$-matrix. Since at least one of the maximal minors of $\varphi_{11}$ is nonzero we must have $r=s$. But then our assumption on $\varphi_{11}$ is contradicted.

Assume now that $r=m+1$, so $\alpha$ is surjective. If $\beta$ is not surjective we get the same contradiction as above. Finally, if $\beta$ is surjective then

$$
\varphi_{11} \sim\left[\begin{array}{cc}
\varphi^{\prime} & 0 \\
\star & \star
\end{array}\right]
$$

Also, $1=\alpha_{0}(\mathscr{E})<(3 / n) \alpha_{1}(\mathscr{E})=3 m / n$ forces $(n / 3)+1<m+1$, so our assumption on $\varphi_{11}$ is contradicted.

Claim 6.11. Let $W$ be the space of morphisms

$$
\varphi:(n-2) \mathcal{O}(-2) \oplus 3 \mathcal{O}(-1) \rightarrow(n-3) \mathcal{O}(-1) \oplus 3 \mathcal{O} .
$$

Let $\Lambda=\left(\lambda_{1}, \lambda_{2}, \mu_{1}, \mu_{2}\right)$ be a polarization satisfying

$$
\lambda_{1}<\mu_{1}<\frac{n-2}{n-3} \lambda_{1}, \quad \frac{3(n-2) \lambda_{1}-1}{2(n-3)}<\mu_{1} .
$$

Equivalently, $\Lambda$ is such that the pair $\left(\lambda_{1}, \mu_{1}\right)$ is in the interior of the triangle with vertices $(0,0),\left(\frac{1}{n}, \frac{1}{n}\right),\left(\frac{1}{n-2}, \frac{1}{n-3}\right)$. Then $\varphi$ is semistable with respect to $\Lambda$ only if 
$\varphi$ is not equivalent to a matrix having one of the forms
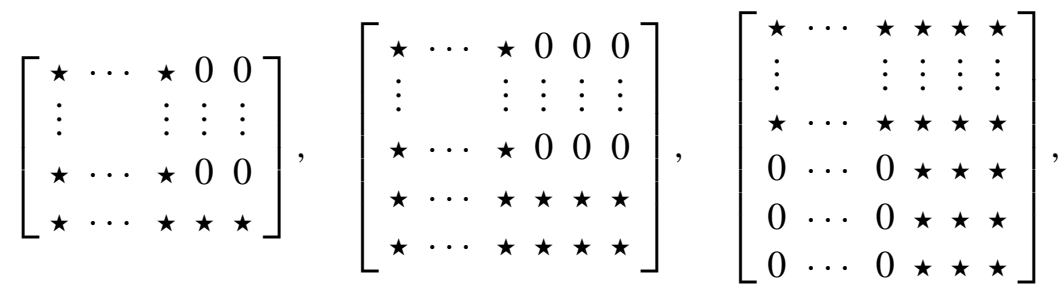

$\left[\begin{array}{llll}\star & \cdots & \star & \star \\ \vdots & & \vdots & \vdots \\ \star & \cdots & \star & \star \\ 0 & \cdots & 0 & \star\end{array}\right]$,

$$
\left[\begin{array}{lllll}
\star & \cdots & \star & \star & \star \\
\vdots & & \vdots & \vdots & \vdots \\
\star & \cdots & \star & \star & \star \\
0 & \cdots & 0 & \star & \star \\
0 & \cdots & 0 & \star & \star
\end{array}\right],
$$

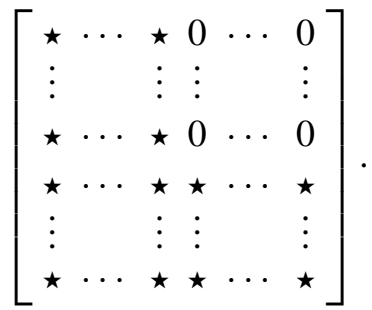

(The zero submatrix in the last matrix has $m$ rows and $n+1-m$ columns, with $1 \leq m \leq n-3$.)

Proof. Using Proposition 3.3 we translate the conditions that $\varphi$ be not equivalent to the above matrices into conditions on $\Lambda$. We arrive at 6 inequalities which, after simplifications, reduce to the inequalities from the claim.

Claim 6.12. Let $\Lambda$ be a polarization as at Claim 6.11. If $4 \leq n \leq 15$ then the morphisms $\varphi$ from Claim 6.9 and Claim 6.10 form a thin constructible subset of $W^{\mathrm{ss}}(G, \Lambda)$.

Proof. First we notice that the morphisms $\varphi$ from Claim 6.9 and Claim 6.10 are in the closed subset of $W^{\mathrm{ss}}(G, \Lambda)$ given by the conditions $\varphi_{12}=0$ and $\operatorname{det}\left(\varphi_{22}\right)=0$. The condition

$$
\varphi_{22} \sim\left[\begin{array}{rrr}
-Y & X & 0 \\
-Z & 0 & X \\
0 & -Z & Y
\end{array}\right]
$$

is a locally closed condition because any orbit with respect to the action of an algebraic group is a locally closed set. The condition $\mathscr{K} \operatorname{er}(\varphi) \simeq \mathcal{O}(-2)$ gives a constructible set as can be seen from the sequel. The condition

$$
\varphi_{11} \nsim\left[\begin{array}{cc}
\varphi^{\prime} & 0 \\
\star & \star
\end{array}\right] \quad \text { with } \operatorname{Ker}\left(\varphi^{\prime}\right) \simeq \mathcal{O}(-3)
$$

gives a constructible set. To see this we only need to prove that the condition $\operatorname{Her}\left(\varphi^{\prime}\right) \simeq \mathcal{O}(-3)$ gives a constructible subset inside the set of $m \times(m+1)$-matrices with entries linear forms. This follows from the following observation: let $G$ be an algebraic group acting on a variety $X$. Let $Y \subset X$ be a constructible subset. Then $G . Y$ is constructible, too. To see this apply Chevalley's theorem, stating that the 
image of a constructible set under an algebraic map is again constructible, to the multiplication map $G \times X \rightarrow X$.

To finish the argument we need to show that the condition $\mathscr{K} e r\left(\varphi^{\prime}\right) \simeq \mathcal{O}(-3)$ gives a constructible set. We represent $\varphi^{\prime}$ by a $m \times(m+1)$-matrix $\alpha=\left(\alpha_{i j}\right)$ with entries in $V^{*}$. Using the notations from Remark 6.5 we see that $\mathscr{K} \operatorname{er}\left(\varphi^{\prime}\right) \simeq \mathcal{O}(-3)$ if and only if $\operatorname{deg}$ (g.c.d. $\left.\left(\alpha_{1}, \ldots, \alpha_{m+1}\right)\right)=m-1$. This, furthermore, is equivalent to the following two conditions:

(i) at least two among $\alpha_{1}, \ldots, \alpha_{m+1}$ are linearly independent;

(ii) the system $\alpha_{i} f_{j}=\alpha_{j} f_{i}, \quad 1 \leq i<j \leq m+1$, has a nontrivial solution $f=$ $\left(f_{1}, \ldots, f_{m+1}\right), f_{j} \in V^{*}$.

In view of Remark 6.5 condition (i) is equivalent to saying that $\mathscr{K e r}(\alpha)$ is not isomorphic to $O(-2)$. This is equivalent to saying that

$$
\alpha \nsim\left[\begin{array}{c}
0 \\
\alpha^{\prime} \\
\vdots \\
\\
0
\end{array}\right] \quad \text { with } \operatorname{det}\left(\alpha^{\prime}\right) \neq 0 .
$$

This condition gives a constructible set because the matrices on the right-hand side form a locally closed subset and the smallest invariant subset containing a locally closed subset must be constructible, as observed above.

Condition (ii) is a closed condition. Indeed, the above system can be written as a linear system with unknowns the coefficients of $f_{j}, 1 \leq j \leq m+1$, and coefficients the coefficients of $\alpha_{i}, 1 \leq i \leq m+1$. Such a linear system has a nontrivial solution if and only if the associated matrix has vanishing maximal minors. These minors are polynomials in the coefficients of $\alpha_{i}, 1 \leq i \leq m+1$, so we get closed conditions on $\alpha$.

\section{Applications to moduli spaces}

Thus far, for certain classes of semistable sheaves $\mathscr{F}$, we have found presentations

$$
\mathscr{E}_{1} \stackrel{\varphi}{\rightarrow} \mathscr{E}_{2} \rightarrow \mathscr{F} \rightarrow 0
$$

with decomposable vector bundles $\mathscr{E}_{1}$ and $\mathscr{E}_{2}$. In this section we will describe some locally closed subsets inside the corresponding moduli spaces $\mathrm{M}_{\mathbb{P}^{2}}(r, \chi)$, defined by means of cohomological conditions as in Remark 2.13. The question we will try to answer is whether such a subset is a good quotient of the set of morphisms $\varphi$ modulo the action by conjugation of Aut $\mathscr{E}_{1} \times$ Aut $\mathscr{E}_{2}$. The difficulty here is that Aut $\mathscr{E}$ is a nonreductive group if $\mathscr{E}$ has a direct summand of the form $\mathcal{O}(a) \oplus \mathcal{O}(b)$ with $a \neq b$.

Whenever we are dealing with a fine moduli space we can show the existence of quotients by using the universal family to construct local sections; compare the 
proof of Proposition 7.6. If the moduli space is not fine we need to have a quotient already constructed as, say, in the main theorem from [Drézet 2000]. We apply this theorem in Proposition 7.12 to describe open dense subsets of $\mathrm{M}_{\mathbb{P}^{2}}(6,4)$ and $\mathrm{M}_{\mathbb{P}^{2}}(8,6)$. At Proposition 7.13 we construct the quotient ad hoc as a fiber bundle over a projective variety.

Unfortunately, Drézet and Trautmann's theory of quotients modulo nonreductive groups is still incomplete. For instance, the main theorem from [Drézet 2000] does not cover the quotients from Claim 4.6. Thus, we are not able to describe as a quotient an open dense subset of $\mathbf{M}_{\mathbb{P}^{2}}(9,6)$. Also, we do not know if quotients exist for morphisms of type $(2,2)$. This accounts for the "question marks" in the table from the introduction.

We begin by recalling the notions of good and geometric quotients. Let $G$ be a linear algebraic group acting on a variety $X$. The action is algebraic; that is, the map $G \times X \rightarrow X$ given by $(g, x) \rightarrow g . x$ is a morphism of varieties.

Definition 7.1. A categorical quotient of $X$ by $G$ is a pair $(Y, \pi)$ where $Y$ is a variety and $\pi: X \rightarrow Y$ is a $G$-equivariant morphism satisfying the following universal property: for any other $G$-equivariant morphism $\eta: X \rightarrow Z$ there exists a unique morphism $\rho: Y \rightarrow Z$ making the diagram commute:

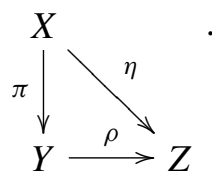

We write $Y=X / / G$. If, in addition, the fibers of $\pi$ are orbits, then $Y$ is called an orbit space and is denoted $X / G$. Categorical quotients are unique up to isomorphism.

Definition 7.2. A good quotient of $X$ by $G$ is a pair $(Y, \pi)$ where $Y$ is a variety and $\pi: X \rightarrow Y$ is a morphism satisfying:

(i) $\pi$ is $G$-equivariant;

(ii) $\pi$ is surjective;

(iii) for any open subset $U \subset Y$ the pull-back map $\pi^{*}$ gives an isomorphism of $\mathrm{O}_{Y}(U)$ onto the ring of regular functions on $\pi^{-1}(U)$ which are constant on the $G$-orbits;

(iv) if $W \subset X$ is closed and $G$-invariant, then $\pi(W)$ is closed;

(v) if $W_{1}, W_{2} \subset X$ are closed, $G$-invariant and disjoint, then $\pi\left(W_{1}\right)$ and $\pi\left(W_{2}\right)$ are also disjoint;

(vi) $\pi$ is affine, i.e., it returns open affine sets to affine sets.

If, in addition, the fibers of $\pi$ are orbits, then $(Y, \pi)$ is called a geometric quotient. 
Remark 7.3. Let $G$ act on $X$ as above and assume the existence of an affine surjective morphism $\pi: X \rightarrow Y$ whose fibers are orbits. Assume that $\pi$ admits local sections; that is, for any $y \in Y$ there is an open neighbourhood $U$ of $y$ and a morphism $\sigma: U \rightarrow X$ satisfying $\pi \circ \sigma=1$. Then $(Y, \pi)$ is a geometric quotient.

Definition 7.2 is important because good quotients are categorical quotients while geometric quotients are orbit spaces:

Proposition 7.4. Let $(Y, \pi)$ be a good quotient of $X$ by $G$. Then:

(i) $(Y, \pi)$ is a categorical quotient;

(ii) $\pi\left(x_{1}\right)=\pi\left(x_{2}\right)$ if and only if $\overline{G . x_{1}}$ intersects $\overline{G \cdot x_{2}}$;

(iii) if the $G$-orbits in $X$ are closed, then $(Y, \pi)$ is an orbit space;

(iv) Let $X_{o}$ denote the subset of points $x \in X$ with G.x closed and of maximal dimension among the $G$-orbits. Then there is an open subset $Y_{o} \subset Y$ such that $\pi^{-1}\left(Y_{o}\right)=X_{o}$ and $\left(Y_{o}, \pi\right)$ is a geometric quotient of $X_{o}$ by $G$.

The main technical tool that we will use in this section is the relative Beilinson complex. Given a variety $X$ and a coherent sheaf $\mathscr{F}$ on $X \times \mathbb{P}^{2}$ there is a sequence

$$
0 \rightarrow \mathscr{C}^{-2} \rightarrow \mathscr{C}^{-1} \rightarrow \mathscr{C}^{0} \rightarrow \mathscr{C}^{1} \rightarrow \mathscr{C}^{2} \rightarrow 0
$$

of sheaves on $X \times \mathbb{P}^{2}$ which is exact, except in the middle, where the cohomology is $\mathscr{F}$. On each fiber $\{x\} \times \mathbb{P}^{2}$ this sequence restricts to the Beilinson complex of the restricted sheaf $\mathscr{F}_{x}$. Let $p: X \times \mathbb{P}^{2} \rightarrow X$ be the projection onto the first component. The sheaves $\mathscr{C}^{i}$ are defined by means of the higher direct images of $\mathscr{F}$ :

$$
\mathscr{C}^{i}=\oplus_{j} R^{j} p_{*}\left(\mathscr{F} \otimes \Omega_{X \times \mathbb{P}^{2} / X}^{j-i}(j-i)\right) \otimes \mathcal{O}_{\mathbb{P}^{2}}(i-j) .
$$

In our applications $\mathscr{F}_{F}$ will be flat over $X$ and its restrictions $\mathscr{F}_{x}$ onto the fibers $\{x\} \times \mathbb{P}^{2}$ will have one-dimensional supports. Thus $H^{2}\left(\mathscr{F}_{x}\right)=0$ for all $x \in X$. From the Base Change Theorem on p. 11 in [Okonek et al. 1980] we get $R^{2} p_{*}(\mathscr{F})=0$. Analogously, the other second direct images occuring above are zero. The relative Beilinson complex now takes the form

$$
0 \rightarrow \mathscr{C}^{-2} \rightarrow \mathscr{C}^{-1} \rightarrow \mathscr{C}^{0} \rightarrow \mathscr{b}^{1} \rightarrow 0
$$

with

$$
\begin{aligned}
\mathscr{C}^{-2} & =p_{*}(\mathscr{F}(-1)) \otimes \mathscr{O}(-2), \\
\mathscr{C}^{-1} & =p_{*}\left(\mathscr{F} \otimes \Omega_{X \times \mathbb{P}^{2} / X}^{1}(1)\right) \otimes \mathscr{O}(-1) \oplus R^{1} p_{*}(\mathscr{F}(-1)) \otimes \mathcal{O}(-2), \\
\mathscr{C}^{0} & =p_{*}(\mathscr{F}) \otimes \mathscr{O} \oplus R^{1} p_{*}\left(\mathscr{F} \otimes \Omega_{X \times \mathbb{P}^{2} / X}^{1}(1)\right) \otimes \mathscr{O}(-1), \\
\mathscr{C}^{1} & =R^{1} p_{*}(\mathscr{F}) \otimes \mathfrak{O} .
\end{aligned}
$$


Proposition 7.6. Let $n \geq 1$ be an integer and let $W$ be the vector space of morphisms $\varphi$ of sheaves on $\mathbb{P}^{2}$ of the form

$$
\mathrm{O}(-2) \oplus(n-1) \mathcal{O}(-1) \stackrel{\varphi}{\rightarrow} n \mathcal{O} .
$$

With the notations from Section 3, assume that the polarization $\Lambda=\left(\lambda_{1}, \lambda_{2}, \mu_{1}\right)$ satisfies $0<\lambda_{1}<1 / n$. Let $W_{o}$ be the open subset of $W^{\text {ss }}(G, \Lambda)$ given by the condition $\operatorname{det}(\varphi) \neq 0$. Then $W_{o}$ admits a geometric quotient modulo $G$ which is isomorphic to the open dense subset of $\mathrm{M}_{\mathbb{P} 2}(n+1, n)$ given by the condition $h^{0}(\mathscr{F}(-1))=0$.

Proof. Consider the coherent sheaf $\widetilde{\mathscr{F}}$ on $W_{o} \times \mathbb{P}^{2}$ given by the exact sequence

$$
\mathfrak{O}_{W_{o}} \otimes \mathcal{O}_{\mathbb{P}^{2}}(-2) \oplus \mathcal{O}_{W_{o}} \otimes(n-1) \mathcal{O}_{\mathbb{P}^{2}}(-1) \stackrel{\Phi}{\rightarrow} n \mathcal{O}_{W_{o} \times \mathbb{P}^{2}} \rightarrow \widetilde{\mathscr{F}}^{\rightarrow} 0 .
$$

On each fiber $\{\varphi\} \times \mathbb{P}^{2}$ this sequence restricts to

$$
0 \rightarrow \mathcal{O}(-2) \oplus(n-1) \mathcal{O}(-1) \stackrel{\varphi}{\rightarrow} n \mathcal{O} \rightarrow \widetilde{\mathscr{F}}_{\varphi} \rightarrow 0 .
$$

According to Claim 4.2 each restriction $\widetilde{\mathscr{F}}_{\varphi}$ is semistable with Hilbert polynomial $P(t)=(n+1) t+n$. As the Hilbert polynomial is independent of $\varphi$, and as the base $W_{o}$ is reduced, the sheaf $\widetilde{\mathscr{F}}$ is flat over $W_{o}$. By Definition 2.5 of a coarse moduli space, $\widetilde{\mathscr{F}}$ gives rise to a morphism

$$
\eta: W_{o} \rightarrow \mathrm{M}_{\mathbb{P}^{2}}(n+1, n),
$$

which sends $\varphi$ to the stable equivalence class of $\widetilde{\mathscr{F}}_{\varphi}$.

By Claim 4.2 the image of $\eta$ is the subset $\mathrm{M}_{o}$ of $\mathrm{M}_{\mathbb{P}^{2}}(n+1, n)$ given by the condition $h^{0}(\mathscr{F}(-1))=0$. By Remark 2.13 this subset is open and, as the moduli space is irreducible, it must be dense.

The fibers of $\eta$ are $G$-orbits. Indeed, an isomorphism $f$ between two cokernels $\mathscr{F}_{1}$ and $\mathscr{F}_{2}$ of $\varphi_{1}$ and $\varphi_{2}$ from $W_{o}$ must fit into a commutative diagram

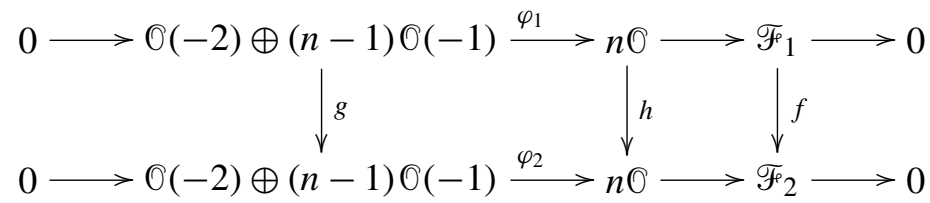

in which $g$ and $h$ are isomorphisms. Here $h$ is defined in such a way as to coincide with $f$ on the level of global sections, while $g$ is the induced map on the kernels.

To prove that $\eta$ is a geometric quotient map it is enough to construct local sections as in Remark 7.3. For this we will use the fact that $\mathbf{M}_{\mathbb{P}^{2}}(n+1, n)$ is a fine moduli space, cf. Proposition 2.10, so it has a universal family. Let $\mathcal{U}$ denote the restriction of the universal family to $\mathrm{M}_{o} \times \mathbb{P}^{2}$. Let $p: \mathrm{M}_{o} \times \mathbb{P}^{2} \rightarrow \mathrm{M}_{o}$ be the projection onto the first component. $U$ is flat over $\mathrm{M}_{o}$ and all its restrictions to the 
fibers of $p$ have Beilinson resolution as in the statement of Claim 4.2. In view of the Base Change Theorem the higher direct images

$$
\begin{aligned}
& p_{*}(U(-1)), \quad R^{1} p_{*}(U(-1)), \quad p_{*}(U), \quad R^{1} p_{*}(U), \\
& p_{*}\left(\mathcal{U} \otimes \Omega_{\mathrm{M}_{o} \times \mathbb{P}^{2} / \mathrm{M}_{o}}^{1}(1)\right), \quad R^{1} p_{*}\left(\mathcal{U} \otimes \Omega_{\mathrm{M}_{o} \times \mathbb{P}^{2} / \mathrm{M}_{o}}^{1}(1)\right)
\end{aligned}
$$

are locally free of ranks $0,1, n, 0, n-1,0$. We cover $\mathrm{M}_{o}$ with open subsets $S$ on which the above sheaves are free. On $S \times \mathbb{P}^{2}$, and relative to fixed trivializations of the higher direct images, the Beilinson complex (7.5) gives the resolution

$$
0 \rightarrow \mathcal{O}_{S} \otimes \mathbb{O}_{\mathbb{P}^{2}}(-2) \oplus \mathcal{O}_{S} \otimes(n-1) \mathcal{O}_{\mathbb{P}^{2}}(-1) \stackrel{\varphi}{\rightarrow} n \mathscr{O}_{S \times \mathbb{P}^{2}} \rightarrow u \rightarrow 0 .
$$

We put $\sigma(x)=\varphi_{x}$ for $x \in S$ and notice that $\sigma: S \rightarrow W_{o}$ is a local section of $\eta$.

The sets $W_{o}$ are nonempty for all $n$. Indeed, it is easy to construct an $n \times(n-1)$ matrix $\psi$ with entries in $V^{*}$ whose maximal minors are linearly independent, and which has the form

$$
\left[\begin{array}{cccc}
\star & \star & \cdots & \star \\
\star & \star & \cdots & \star \\
0 & \star & \cdots & \star \\
\vdots & \vdots & \ddots & \vdots \\
0 & 0 & \cdots & \star
\end{array}\right] .
$$

For example,

$$
\left[\begin{array}{llll}
Y & Z & Y & Z \\
X & 0 & 0 & 0 \\
0 & Y & 0 & 0 \\
0 & 0 & Z & 0 \\
0 & 0 & 0 & X
\end{array}\right]
$$

is such a matrix for $n=5$. But it now becomes clear that the following matrix is semistable and has nonzero determinant:

$$
\left[\begin{array}{cc}
X^{2} & \psi \\
0 & \psi
\end{array}\right]
$$

The existence of the geometric quotient $W_{o} / G$ can be put into a broader context if we realize that $W^{\text {ss }}(G, \Lambda)$ itself has a geometric quotient, as J.-M. Drézet pointed out to the author:

Proposition 7.7. Let $W^{\mathrm{ss}}(G, \Lambda)$ be the set of morphisms of sheaves on $\mathbb{P}^{2}$,

$$
\mathcal{O}(-2) \oplus(n-1) \mathcal{O}(-1) \stackrel{\varphi}{\rightarrow} n \mathcal{O},
$$

which are semistable with respect to a polarization $\Lambda$ satisfying $0<\lambda_{1}<1 / n$. Then there exists a geometric quotient $W^{\mathrm{ss}}(G, \Lambda) / G$ which is a fiber bundle with fiber $\mathbb{P}^{3 n+2}$ and base a projective variety of dimension $n^{2}-n$. 
Proof. Write $\varphi$ as a pair $\left(\varphi_{1}, \varphi_{2}\right)$, where $\varphi_{1}$ is an $n \times 1$-matrix with entries in $S^{2} V^{*}$, while $\varphi_{2}$ is an $n \times(n-1)$-matrix with entries in $V^{*}$. Let $W_{i}$ denote the vector space of matrices $\varphi_{i}$.

The reductive group $G_{2}=\mathrm{GL}(n-1) \times \mathrm{GL}(n) / k^{*}$ acts on $W_{2}$ by conjugation. Here $k^{*}$ is embedded as the subgroup of homotheties. The only admissible polarization on $W_{2}$ is $(1 /(n-1), 1 / n)$ and, since $n-1$ and $n$ are mutually prime, equality cannot be achieved in Proposition 3.3. This shows that the set of semistable points $W_{2}^{\text {ss }}$ for the action of $G_{2}$ coincides with the set of stable points. By classical geometric invariant theory there is a geometric quotient $W_{2}^{\text {ss }} / G_{2}$ which is a projective variety of dimension $n^{2}-n$. We denote it by $N$.

We view $W$ as a trivial bundle with fiber $W_{1}$ and base $W_{2}$. Let $U$ be the trivial bundle on $W_{2}$ with fiber the space of $(n-1) \times 1$-matrices with entries in $V^{*}$. We consider the morphism of bundles $f: U \rightarrow W$ given at every point $\varphi_{2}$ by leftmultiplication with $\varphi_{2}$. It is easy to see that $f$ is injective at every semistable point $\varphi_{2}$, hence the restriction of $\operatorname{Coker}(f)$ to $W_{2}^{\text {ss }}$ is a vector bundle of rank $3 n+3$, denoted by $E . \mathbb{P}(E)$ carries a $G_{2}$ action which is compatible with the action on $W_{2}^{\text {ss }}$. At Lemma 8.1 below we will prove that for any $\varphi_{2} \in W_{2}^{\text {ss }}$ the isotropy group $\operatorname{Stab}_{G_{2}}\left(\varphi_{2}\right)$ is trivial, so it acts trivially on $\mathbb{P}\left(E_{\varphi_{2}}\right)$. It follows that $\mathbb{P}(E)$ descends to a fiber bundle $F$ on $N$, see 4.2.15 in [Huybrechts and Lehn 1997].

We notice that the semistability conditions on $\varphi$ read as follows: $\varphi_{2}$ is in $W_{2}^{\text {ss }}$ and $\varphi_{1}^{\prime} \neq 0$ for all $\varphi^{\prime}$ in the same $G$-orbit as $\varphi$. In other words, $W^{\text {ss }}(G, \Lambda)$ can be identified with the complement of $\mathscr{I} m(f)$ inside $W_{1 \mid W_{2}^{\text {ss. }}}$. The map

$$
W_{1 \mid W_{2}^{\text {ss }}} \backslash \Phi_{m}(f) \rightarrow \mathbb{P}(E)
$$

admits local sections because $E$ is a bundle. In view of Remark 7.3 this map is a geometric quotient modulo the action of the subgroup of $G$ given by the conditions $h_{1}=1, g_{2}=1$, see the notations preceeding Definition 3.1. Combining this with the fact that the map $\mathbb{P}(E) \rightarrow F$ is a geometric quotient modulo $G_{2}$, we easily deduce that the map $W^{\text {ss }}(G, \Lambda) \rightarrow F$ is a geometric quotient modulo $G$.

Our construction is similar to, though much less elegant than, the construction from 10.2 in [Drézet and Trautmann 2003] which addresses morphisms on $\mathbb{P}^{n}$ of the form

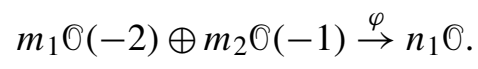

The polarization satisfies $0<\lambda_{1}<\lambda_{\min }$, where $\lambda_{\min }$ is the smallest positive number such that for $\lambda_{1}$ varying in the interval $\left(0, \lambda_{\min }\right)$ the set of semistable points remains unchanged. In the context of the above proposition $\lambda_{\min }=1 / n$. They show that if certain conditions on the integers $m_{1}, m_{2}, n_{1}$ are satisfied, then there exists a geometric quotient which is a Grassmann bundle $\operatorname{Grass}\left(m_{1}, p_{*} \mathscr{E}(2)\right)$ with base $N=W_{2}^{\text {ss }} / G_{2}$. Here $p: N \times \mathbb{P}^{2} \rightarrow N$ is the projection onto the first component and $\mathscr{E}$ 
is the universal sheaf on $N \times \mathbb{P}^{2}$ that restricts to $\operatorname{Coker}\left(\varphi_{2}\right)$ on each fiber $\left\{\left[\varphi_{2}\right]\right\} \times \mathbb{P}^{2}$. One of their conditions, having to do with the injectivity of $\varphi_{2}$ regarded as map from $m_{2} \mathcal{O}(-1)$ to $n_{1} \mathbb{O}$, is $n_{1} \geq n m_{2}$. Thus Drézet and Trautmann's construction addresses only the case $n=2$ of the above proposition.

There is yet another, more direct way of constructing the quotient in the case $n=2$. The semistability conditions on a morphism $\varphi: \mathcal{O}(-2) \oplus \mathcal{O}(-1) \rightarrow 20$ read: $\operatorname{det}(\varphi) \neq 0$ and $\varphi_{12}, \varphi_{22}$ are linearly independent in $V^{*}$. The map

$$
W^{\mathrm{ss}}(G, \Lambda) \rightarrow \operatorname{Grass}\left(2, V^{*}\right) \times \mathbb{P}\left(S^{3} V^{*}\right) \simeq \mathbb{P}^{2} \times \mathbb{P}\left(S^{3} V^{*}\right)
$$

given by

$$
\varphi \rightarrow\left(\operatorname{span}\left(\varphi_{12}, \varphi_{22}\right),\langle\operatorname{det}(f)\rangle\right)
$$

has fibers $G$-orbits and has image the universal cubic

$$
C=\left\{(x,\langle f\rangle) \in \mathbb{P}^{2} \times \mathbb{P}\left(S^{3} V^{*}\right), \quad f(x)=0\right\} .
$$

It was first noticed in [Maican 2000] that the map $W^{\mathrm{ss}}(G, \Lambda) \rightarrow C$ has local sections: Choose a point $(x,\langle f\rangle)$ in $C$, say $x=(0: 0: 1)$. As $f$ does not contain the monomial $Z^{3}$, there are unique quadratic polynomials $q_{1}(X, Y, Z)$ and $q_{2}(X, Z)$ such that $f=q_{1} Y-q_{2} X$. We put

$$
\sigma(x,\langle f\rangle)=\left[\begin{array}{ll}
q_{1} & X \\
q_{2} & Y
\end{array}\right] .
$$

Since all processes involved in defining $\sigma$ are algebraic, we see that $\sigma$ extends to a section of the map $W^{\text {ss }}(G, \Lambda) \rightarrow C$ defined on a neighbourhood of $(x,\langle f\rangle)$. Thus $W^{\text {ss }}(G, \Lambda) / G \simeq C$. A more sophisticated proof of this isomorphism can be found in [Freiermuth 2000].

In the simplest case $n=1, W^{\mathrm{ss}}(G, \Lambda)$ is just the set of nonzero morphisms $\mathcal{O}(-2) \rightarrow \mathcal{O}$ and $W^{\text {ss }}(G, \Lambda) / G$ is $\mathbb{P}\left(S^{2} V^{*}\right)$.

As noticed, in the cases $n=1,2$ we have $W_{o}=W^{\mathrm{ss}}(G, \Lambda)$, hence $W_{o} / G$ is complete, hence the set $\mathrm{M}_{o}$ from Proposition 7.6 is complete, hence $\mathrm{M}_{o}$ is the entire moduli space. We have obtained the well-known fact that every semistable sheaf on $\mathbb{P}^{2}$ with Hilbert polynomial $P(t)=2 t+1$ is the structure sheaf of a conic; in other words $\mathrm{M}_{\mathbb{P}^{2}}(2,1) \simeq \mathbb{P}\left(S^{2} V^{*}\right)$. In the case $n=3$ we have rediscovered one of Le Potier's result from [Le Potier 1993] to the effect that $\mathbf{M}_{\mathbb{P}^{2}}(3,2)$ is isomorphic to the universal cubic.

If $n \geq 3 W_{o}$ is a proper open subset of the set of semistable points, hence $W_{o} / G$ is not complete, hence $\mathbf{M}_{o}$ is a proper open subset of $\mathbf{M}_{\mathbb{P}^{2}}(n+1, n)$. Indeed, it is 
easy to construct semistable morphisms with zero determinant; for example, in the case $n=3$,

$$
\left[\begin{array}{ccc}
0 & X & Y \\
X Y & Z & 0 \\
-X^{2} & 0 & Z
\end{array}\right] .
$$

Thus, the most we can say at this time is this:

Corollary 7.8. For $n \geq 3$ the projective varieties $W^{\mathrm{ss}}(G, \Lambda) / G$ and $\mathrm{M}_{\mathbb{P}^{2}}(n+1, n)$ are birational.

Proof. From Definition 7.2(iv) we see that the image of $W_{o}$ under the quotient map

$$
W^{\mathrm{ss}}(G, \Lambda) \rightarrow W^{\mathrm{ss}}(G, \Lambda) / G
$$

is an open set $U$. In fact, $W_{o}$ is the preimage of $U$. Clearly, the properties from Definition 7.2 are satisfied for the map $W_{o} \rightarrow U$. This proves that $W_{o} / G \simeq U$ and so we have isomorphic open dense subsets of $W^{\text {ss }}(G, \Lambda) / G$ and of $\mathrm{M}_{\mathbb{P}^{2}}(n+1, n)$.

The same proof as in Proposition 7.6 can be used to show that for all fine moduli spaces $\mathrm{M}_{\mathbb{P}^{2}}(r, \chi)$ occuring in Sections 4-6 the locally closed subsets described by cohomological conditions are geometric quotients $W_{o} / G$ of the corresponding sets $W_{o} \subset W^{\text {ss }}(G, \Lambda)$. We have summarized the results in the table from the introduction. For the quotients in Section 5 we should mention that $\varphi_{x}$ depends in an algebraic manner on the maps from the Beilinson complex of $u_{x}$, hence it depends in an algebraic manner on $x$; see Proposition 7.14 for the details.

The assumption that $\mathrm{M}_{\mathbb{P} 2}(r, \chi)$ be fine, or equivalently that a universal family exist, is needed for the construction of the local sections of $\eta$. The proof of Proposition 7.6 does not apply if the moduli space is not fine because, according to Theorem 2.11, there is no universal family on any open subset of such a moduli space.

Two of the quotients from Sections 5 and 6 have very concrete descriptions. First we consider the case $n=1$ from Claim 5.3. The set of morphisms

$$
\varphi: \mathcal{O}(-3) \oplus \mathbb{O}(-1) \rightarrow 20
$$

semistable with respect to a polarization satisfying $0<\lambda_{1}<1 / 2$ is characterized by the conditions $\operatorname{det}(\varphi) \neq 0$ and $\varphi_{12}, \varphi_{22}$ are linearly independent in $V^{*}$. The same discussion as in the case $n=2$ of Proposition 7.6 shows that the geometric quotient $W^{\mathrm{ss}}(G, \Lambda) / G$ is isomorphic to the universal quartic in $\mathbb{P}^{2} \times \mathbb{P}\left(S^{4} V^{*}\right)$. From Claim 5.3 we get:

Corollary 7.9. The subset of $\mathrm{M}_{\mathbb{P}^{2}}(4,1)$ given by the conditions $h^{0}(\mathscr{F}(-1))=0$ and $h^{1}(\mathscr{F})=1$ is closed and, equipped with its canonical reduced structure, it is isomorphic to the universal quartic in $\mathbb{P}^{2} \times \mathbb{P}\left(S^{4} V^{*}\right)$. 
Now consider the simplest case $n=4$ from Claim 6.6. It concerns morphisms

$$
\varphi: 2 \mathcal{O}(-2) \oplus 3 \mathcal{O}(-1) \rightarrow \mathcal{O}(-1) \oplus 30
$$

satisfying the conditions: $\varphi_{12}=0, \varphi_{11}$ has linearly independent entries in $V^{*}$, $\varphi_{21}^{\prime} \neq 0$ for any $\varphi^{\prime}$ in the same orbit as $\varphi, \varphi_{22}$ is equivalent to the matrix

$$
\left[\begin{array}{rrr}
-Y & X & 0 \\
-Z & 0 & X \\
0 & -Z & Y
\end{array}\right] .
$$

Let $f=0$ be the equation of the support of $\mathscr{F}$. To be precise,

$$
f=\left[\begin{array}{lll}
Z & -Y & X
\end{array}\right] \varphi_{21}\left[\begin{array}{r}
-X_{2} \\
X_{1}
\end{array}\right], \quad \text { where } \quad \varphi_{11}=\left[\begin{array}{ll}
X_{1} & X_{2}
\end{array}\right]
$$

and $\varphi_{22}$ is assumed to be the above $3 \times 3$-matrix. We consider the $G$-invariant map

$$
W_{o} \rightarrow \operatorname{Grass}\left(2, V^{*}\right) \times \mathbb{P}\left(S^{4} V^{*}\right) \simeq \mathbb{P}^{2} \times \mathbb{P}\left(S^{4} V^{*}\right)
$$

given by

$$
\varphi \rightarrow\left(\operatorname{span}\left\{X_{1}, X_{2}\right\},\langle f\rangle\right) .
$$

Its image is the universal quartic $Q$. To prove that the map $W_{o} \rightarrow Q$ is a geometric quotient, we will construct local sections. We fix a point $\left(\operatorname{span}\left\{X_{1}, X_{2}\right\},\langle f\rangle\right)$ in $Q$. We complete $\left\{X_{1}, X_{2}\right\}$ to a basis $\left\{X_{1}, X_{2}, X_{3}\right\}$ of $V^{*}$. Relative to this basis $f$ can be uniquely written as

$$
f\left(X_{1}, X_{2}, X_{3}\right)=-X_{2} f_{1}\left(X_{1}, X_{2}, X_{3}\right)+X_{1} f_{2}\left(X_{1}, X_{3}\right) .
$$

Now $f_{1}$ and $f_{2}$ can each be uniquely written as

$$
\begin{aligned}
& f_{1}=Z q_{11}(X, Y, Z)-Y q_{21}(X, Y)+X q_{31}(X), \\
& f_{2}=Z q_{12}(X, Y, Z)-Y q_{22}(X, Y)+X q_{32}(X) .
\end{aligned}
$$

We put

$$
\sigma\left(\operatorname{span}\left\{X_{1}, X_{2}\right\},\langle f\rangle\right)=\left[\begin{array}{rrrrr}
X_{1} & X_{2} & 0 & 0 & 0 \\
q_{11} & q_{12} & -Y & X & 0 \\
q_{21} & q_{22} & -Z & 0 & X \\
q_{31} & q_{32} & 0 & -Z & Y
\end{array}\right] .
$$

Since all processes involved in defining $\sigma$ are algebraic, we see that $\sigma$ extends to a local section defined on an open subset of $Q$. From Claim 6.6 we get:

Corollary 7.10. The subset of $\mathrm{M}_{\mathbb{P}^{2}}(4,3)$ given by the conditions $h^{0}(\mathscr{F}(-1))=1$ and $h^{1}(\mathscr{F})=0$ is closed and, equipped with its canonical reduced structure, is isomorphic to the universal quartic in $\mathbb{P}^{2} \times \mathbb{P}\left(S^{4} V^{*}\right)$. 
We now turn to the moduli spaces $\mathrm{M}_{\mathbb{P}^{2}}(r, \chi)$ for which $r$ and $\chi$ are not mutually prime. As we shall see, if we knew the existence of the quotient $W_{o} / / G$, then we could prove that this quotient is isomorphic to the corresponding subvariety of the moduli space. We know the existence of the quotients only in two cases: for the situation in Claim 4.3(i) and for $n=3$ in Claim 5.3. In the first case we will use a theorem of Drézet:

Let $m_{1}, m_{2}, n_{1}$ be integers and consider morphisms of sheaves on $\mathbb{P}^{n}$ of the form

$$
m_{1} \bigcirc(-2) \oplus m_{2} \bigcirc(-1) \stackrel{\varphi}{\rightarrow} n_{1} \bigcirc .
$$

We recall from Section 3 that a polarization in this context is a triple $\Lambda=\left(\lambda_{1}, \lambda_{2}, \mu_{1}\right)$ of positive numbers satisfying the relations $m_{1} \lambda_{1}+m_{2} \lambda_{2}=n_{1} \mu_{1}=1$. Theorem 6.4 from [Drézet 2000] gives sufficient conditions on $\Lambda$ which assure the existence of a good quotient. Below we state part two of the theorem formulated in the particular case of the projective plane which is of interest to us:

There exists a good quotient $W^{\text {ss }}(G, \Lambda) / / G$, which is a projective variety, if the following four inequalities are fulfilled:

$$
\begin{array}{rlrl}
\lambda_{2} & <\frac{3}{n_{1}}, \quad \lambda_{2}>\frac{3 m_{1}+n_{1}}{3 m_{1} n_{1}+n_{1} m_{2}}, \\
m_{2} \lambda_{2} & >1-\frac{3 m_{1}}{n_{1}\left(3 m_{1}-1\right)} & \text { if } m_{1} \leq 3, \\
m_{2} \lambda_{2} & >1-\frac{3 m_{1}}{8 n_{1}} & \text { if } m_{1}>3 .
\end{array}
$$

Taking $m_{1}=2, m_{2}=n-2, n_{1}=n$ these conditions become

$$
\lambda_{1}<\frac{6}{n(n+4)}, \quad \lambda_{1}<\frac{3}{5 n} .
$$

Corollary 7.11. Let $3 \leq n \leq 7$ be an integer and let $W^{\mathrm{ss}}(G, \Lambda)$ be the space of morphisms of sheaves on $\mathbb{P}^{2}$ of the form

$$
2 \mathcal{O}(-2) \oplus(n-2) \mathcal{O}(-1) \stackrel{\varphi}{\rightarrow} n \mathcal{O}
$$

that are semistable with respect to a polarization $\Lambda$ satisfying $1 /(2 n)<\lambda_{1}<1 / n$. Then there exists a good quotient $W^{\mathrm{ss}}(G, \Lambda) / / G$, which, moreover, is a projective variety.

Proof. If

$$
\frac{1}{2 n}<\lambda_{1}<\frac{6}{n(n+4)}
$$

the statement follows from Drézet's theorem. To conclude the proof we only need observe that $W^{\text {ss }}(G, \Lambda)$ does not change when $\lambda_{1}$ varies in the interval $\left(\frac{1}{2 n}, \frac{1}{n}\right)$. 
Proposition 7.12. For $3 \leq n \leq 6$ let $W_{o}$ be the subset of $W^{\text {ss }}(G, \Lambda)$ from Corollary 7.11 given by the condition $\operatorname{det}(\varphi) \neq 0$. For $n=2$ let $W_{o}$ be the space of injective morphisms $20(-2) \rightarrow 20$. Then $W_{o}$ admits a good quotient modulo $G$, which is isomorphic to the open dense subset of $\mathrm{M}_{\mathbb{P}^{2}}(n+2, n)$ given by the conditions

$$
h^{0}(\mathscr{F}(-1))=0, \quad h^{1}(\mathscr{F})=0, \quad h^{1}\left(\mathscr{F} \otimes \Omega^{1}(1)\right)=0 .
$$

In particular, the projective varieties $W^{\mathrm{ss}}(G, \Lambda) / / G$ and $\mathrm{M}_{\mathbb{P} 2}(n+2, n)$ are birational.

Proof. The good quotient $W_{o} / / G$ is an open dense subset of $W^{\text {ss }}(G, \Lambda) / / G$. The latter exists by Corollary 7.11 when $n \geq 3$ and by classical geometric invariant theory when $n=2$. The map

$$
\eta: W_{o} \rightarrow \mathrm{M}_{\mathbb{P}^{2}}(n+2, n)
$$

can be constructed as in Proposition 7.6 and has image the open subset $\mathbf{M}_{o}$ described by the cohomological conditions from the proposition. By the universal property 7.4(i) of a good quotient, $\eta$ factors through a morphism

$$
\rho: W_{o} / / G \rightarrow \mathrm{M}_{o} .
$$

If $n$ is even the injectivity of $\rho$ is not as straightforward as in Proposition 7.6 because the fibers of $\eta$ may not be $G$-orbits, as there may occur properly semistable sheaves. We will prove the injectivity only in the case $n=2$, the cases $n=4$ and $n=6$ being analogous:

Let $\left[\varphi_{1}\right]$ and $\left[\varphi_{2}\right]$ be in $W_{o} / / G$ and assume that $\mathscr{F}_{1}=\operatorname{Coker}\left(\varphi_{1}\right)$ and $\mathscr{F}_{2}=$ $\operatorname{Coker}\left(\varphi_{2}\right)$ are properly semistable and stable equivalent. Thus $\mathscr{F}_{1}$ and $\mathscr{F}_{2}$ have the same terms in their Jordan-Hölder filtrations, say $\mathscr{A}_{1}$ and $\mathscr{A}_{2}$. According to the discussion preceeding Corollary 7.8, $\mathscr{A}_{i}$ are cokernels of maps $\alpha_{i}: \mathcal{O}(-2) \rightarrow \mathbb{O}$. It is easy to see that, modulo the action of $G, \varphi_{1}$ and $\varphi_{2}$ are equivalent to matrices

$$
\psi_{1}=\left[\begin{array}{cc}
\alpha_{1} & \beta_{1} \\
0 & \alpha_{2}
\end{array}\right], \quad \text { respectively } \quad \psi_{2}=\left[\begin{array}{cc}
\alpha_{1} & \beta_{2} \\
0 & \alpha_{2}
\end{array}\right] .
$$

We consider the one-parameter subgroup $\lambda$ of $G$ given by

$$
\lambda(t)=\left(\left[\begin{array}{ll}
t & 0 \\
0 & 1
\end{array}\right],\left[\begin{array}{ll}
t & 0 \\
0 & 1
\end{array}\right]\right) .
$$

We have

$$
\lambda(t) . \psi_{i}=\left[\begin{array}{cc}
\alpha_{1} & t \beta_{i} \\
0 & \alpha_{2}
\end{array}\right], \quad \text { forcing } \quad \lim _{t \rightarrow 0} \lambda(t) . \psi_{i}=\left[\begin{array}{cc}
\alpha_{1} & 0 \\
0 & \alpha_{2}
\end{array}\right],
$$

which we denote by $\psi$. From Proposition 7.4(ii) we get $\left[\psi_{i}\right]=[\psi]$, so $\left[\varphi_{i}\right]=[\psi]$, so $\rho$ is injective. 
To finish the proof we only need observe that $\mathrm{M}_{o}$ is smooth. At points represented by stable sheaves this is already known from Theorem 2.12. In general, applying the long exact sequence of Ext groups to the exact sequence from Claim 4.3(i), we deduce that for all $\mathscr{F}$ in $\mathrm{M}_{o}$ we have $\operatorname{Ext}^{2}(\mathscr{F}, \mathscr{F})=0$. According to Grothendieck's Criterion, this gives smoothness at the point in the moduli space represented by $\mathscr{F}$.

Thus far $\rho$ is a bijective morphism onto a normal variety. From Zariski's Main Theorem we conclude that $\rho$ is an isomorphism.

We notice that another way of proving that $\rho$ is an isomorphism, which avoids Grothendieck's Criterion of smoothness and Zariski's Main Theorem, is exhibited in the proof of Proposition 7.14.

We do not know if the birational maps constructed above are isomorphisms. The subsets $\mathbf{M}_{o}$ are open, proper subsets of $\mathbf{M}_{\mathbb{P}^{2}}(n+2, n)$ because $W_{o}$ are proper subsets of the sets of semistable points. For example, in the case $n=2$, the following matrices are semistable but have zero determinant:

$$
\left[\begin{array}{ll}
X_{1} Y_{1} & X_{1} Y_{2} \\
X_{2} Y_{1} & X_{2} Y_{2}
\end{array}\right]
$$

where $X_{1}, X_{2}$ are linearly independent in $V^{*}$ and same for $Y_{1}, Y_{2}$.

Proposition 7.13. For $n=1,2,3$ let $W^{\mathrm{ss}}(G, \Lambda)$ be the set of morphisms of sheaves on $\mathbb{P}^{2}$ of the form

$$
\mathcal{O}(-3) \oplus n \mathcal{O}(-1) \stackrel{\varphi}{\rightarrow}(n+1) \mathcal{O},
$$

which are semistable with respect to a polarization $\Lambda$ satisfying $0<\lambda_{1}<1 / n+1$. Then there exists a geometric quotient $W^{\mathrm{ss}}(G, \Lambda) / G$ which is a fiber bundle with fiber $\mathbb{P}^{4 n+9}$ and base a projective variety of dimension $n^{2}+n$.

The proof is the same as in Proposition 7.7. The injectivity of $f$ is clear in the cases $n=1,2$ and follows from remark Remark 5.5 in the case $n=3$.

Proposition 7.14. Let $W_{o}$ be the open subset of $W^{\mathrm{ss}}(G, \Lambda)$ from Proposition 7.13 given by the condition $\operatorname{det}(\varphi) \neq 0$. Then $W_{o}$ admits a geometric quotient modulo $G$ which is isomorphic to the locally closed subset of $\mathrm{M}_{\mathbb{P}^{2}}(n+3, n)$ given by the conditions $h^{0}(\mathscr{F}(-1))=0$ and $h^{1}(\mathscr{F})=1$, and equipped with its canonical reduced structure.

Proof. The cases of the fine moduli spaces $\mathbf{M}_{\mathbb{P}^{2}}(4,1)$ and $\mathbf{M}_{\mathbb{P}^{2}}(5,2)$ were discussed earlier. Assume now that $n=3$. Let $X$ be the subset of $\mathbf{M}_{\mathbb{P}^{2}}(6,3)$ described by the cohomological conditions from the proposition.

As in Proposition 7.6, there is a morphism $\eta: W_{o} \rightarrow X$ associated to a flat family on $W_{o}$ and which factors through a morphism $\rho: W_{o} / G \rightarrow X$. From Remark 5.4 
we know that all sheaves from $X$ are stable, so we can repeat the argument from Proposition 7.6 proving that the fibers of $\eta$ are $G$-orbits. Thus $\rho$ is bijective.

To prove that $\rho$ is an isomorphism we will construct its inverse. Recall from Section 2 that $\mathrm{M}_{\mathbb{P}^{2}}(6,3)$ is the good quotient of a certain open subset $R$ inside a quotient scheme, modulo the action of $\operatorname{SL}(V)$. There is a locally closed subvariety $S$ of $R$, invariant under the action of the special linear group, such that $X=S / / \operatorname{SL}(V)$. The existence of $S$ follows from remark 3.4 .3 on p. 54 in [Newstead 1978] and from the fact that in characteristic zero reductive groups are linearly reductive (p. 50 in the same reference). In fact $S$ is the preimage of $X$ under the quotient map $R \rightarrow \mathrm{M}_{\mathbb{P}^{2}}(6,3)$. Let $\tau: S \rightarrow X$ denote the quotient map.

Let $U$ be the restriction to $S \times \mathbb{P}^{2}$ of the universal quotient family on $R \times \mathbb{P}^{2}$. Let $p: S \times \mathbb{P}^{2} \rightarrow S$ be the projection onto the first component. For an arbitrary point in $s \in S$ we denote by $\mathcal{U}_{s}$ the restriction $\mathcal{U}_{\mid\{s\} \times \mathbb{P}^{2}}$. From Claim 5.3 we know that $u_{s}$ has a resolution

$$
0 \rightarrow \mathcal{O}(-3) \oplus 3 \mathcal{O}(-1) \stackrel{\varphi}{\rightarrow} 40 \rightarrow u_{s} \rightarrow 0
$$

In fact, with the notations from Section 5, we have

$$
\varphi_{12}=\rho_{32}, \quad \varphi_{11}=-\rho_{31} \psi_{12}^{-1}\left[\begin{array}{c}
X \\
Y \\
Z
\end{array}\right]
$$

Each $\varkappa_{s}$ is the middle cohomology of a Beilinson complex

$$
0 \rightarrow 30(-2) \oplus 30(-1) \rightarrow 30(-1) \oplus 40 \rightarrow 0 \rightarrow 0
$$

and $\rho_{32}, \rho_{31}, \psi_{12}$ depend algebraically on the maps in this complex. We put $\varsigma(s)=$ $\varphi$ and we claim that $\varsigma$ can be extended to a morphism from a neighbourhood $S_{o}$ of $s$ in $S$ to $W_{o}$.

To see this we proceed as in the proof of Proposition 7.6. The higher direct image sheaves

$$
\begin{gathered}
p_{*}(\mathcal{U}(-1)), \quad R^{1} p_{*}(\mathcal{U}(-1)), \quad p_{*}(\mathcal{U}), \quad R^{1} p_{*}(\mho), \\
p_{*}\left(U \otimes \Omega_{S \times \mathbb{P}^{2} / S}^{1}(1)\right), \quad R^{1} p_{*}\left(U \otimes \Omega_{S \times \mathbb{P}^{2} / S}^{1}(1)\right)
\end{gathered}
$$

are locally free of ranks $0,3,4,1,3,3$. They are free on an open neighbourhood $S_{o}$ of $s$. Thus $\rho_{32}, \rho_{31}, \psi_{12}$ can be made to depend algebraically on the point in $S_{o}$. This allows us to define $\varsigma$ on $S_{o}$.

We now cover $S$ with such open sets $S_{o}$ and we notice that the locally defined maps $\pi \circ \varsigma$ glue together to a globally defined morphism $\sigma: S \rightarrow W_{o} / G$ making 
the diagram commute:

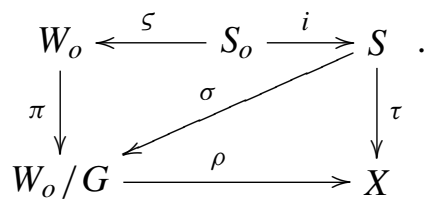

Indeed, if $\varsigma_{1}$ and $\varsigma_{2}$ are defined on two distinct neighbourhoods of $s$, then $\varsigma_{1}(s)$ and $\varsigma_{2}(s)$ are in the same $G$-orbit.

Finally, we observe that $\sigma$ is constant on the fibers of $\tau$. This is so because if $\tau\left(s_{1}\right)=\tau\left(s_{2}\right)$, then the corresponding sheaves $U_{s_{1}}$ and $\vartheta_{s_{2}}$ are isomorphic, so their Beilinson resolutions are equivalent, and $\varsigma_{1}\left(s_{1}\right)$ and $\varsigma_{2}\left(s_{2}\right)$ are in the same $G$-orbit. Here $\varsigma_{i}$ is defined on a neighbourhood of $s_{i}$.

By the universal property 7.4(i) of a good quotient, the map $\sigma$ factors through a morphism from $X$ to $W_{o} / G$. This is the desired inverse of $\rho$.

The above proof could be carried out for all locally closed subsets $X \subset \mathrm{M}_{\mathbb{P}^{2}}(r, \chi)$ occuring at Claim 4.3, Claim 4.5(ii), Claim 4.7, Claim 4.8, provided that we knew the existence of the quotients $W_{o} / / G$. In all cases we would get the isomorphism $X \simeq W_{o} / / G$. Unfortunately, we do not know how to prove the existence of $W_{o} / / G$ when $r$ and $\chi$ are not mutually prime in each of the above cases. We should mention that an essential ingredient in the proof is the fact that all sheaves from $S$ have the same kind of Beilinson complex. This is satisfied because the cohomological conditions defining $X$ are closed under stable equivalence. This fact is easy to check in each case. To give the flavor of the argument we will just check the case $n=6$ from Claim 4.3(i): assume that $\mathscr{G}$ is stable equivalent to $\mathscr{F}_{F}$ and that $\mathscr{F}_{F}$ has resolution

$$
0 \rightarrow 20(-2) \oplus 40(-1) \rightarrow 60 \rightarrow \mathscr{F} \rightarrow 0 .
$$

Assume that $\mathscr{F}$ is properly semistable, so it fits into an exact sequence

$$
0 \rightarrow \mathscr{F}_{1} \rightarrow \mathscr{F} \rightarrow \mathscr{F}_{2} \rightarrow 0
$$

with $\mathscr{F}_{1}$ and $\mathscr{F}_{2}$ in $\mathrm{M}_{\mathbb{P}^{2}}(4,3)$. From $h^{1}(\mathscr{F})=0$ and $h^{2}\left(\mathscr{F}_{1}\right)=0$ we get $h^{1}\left(\mathscr{F}_{2}\right)=0$. Analogously, from $h^{1}\left(\mathscr{F} \otimes \Omega^{1}(1)\right)=0$ and from $h^{2}\left(\mathscr{F}_{1} \otimes \Omega^{1}(1)\right)=0$ we get $h^{1}\left(\mathscr{F}_{2} \otimes\right.$ $\left.\Omega^{1}(1)\right)=0$. We cannot have $h^{0}\left(\mathscr{F}_{2}(-1)\right)>0$ because, in view of Claim 6.6, this would force $h^{1}\left(\mathscr{F}_{2} \otimes \Omega^{1}(1)\right)=1$. Thus $h^{0}\left(\mathscr{F}_{2}(-1)\right)=0$. From $h^{0}(\mathscr{F}(-1))=0$ we immediately also get $h^{0}\left(\mathscr{F}_{1}(-1)\right)=0$. In conclusion, both $\mathscr{F}_{1}$ and $\mathscr{F}_{2}$ satisfy the hypotheses of Claim 4.2. We arrive at the resolutions

$$
0 \rightarrow \mathscr{O}(-2) \oplus 20(-1) \rightarrow 30 \rightarrow \mathscr{F}_{i} \rightarrow 0 .
$$

By hypothesis $G$ is an extension

$$
0 \rightarrow \mathscr{F}_{1} \rightarrow \mathscr{G} \rightarrow \mathscr{F}_{2} \rightarrow 0
$$


possibly with $\mathscr{F}_{1}$ and $\mathscr{F}_{2}$ interchanged. By the "horseshoe lemma" the resolutions of $\mathscr{F}_{1}$ and $\mathscr{F}_{2}$ can be combined to give a resolution for $\mathscr{G}$ of the same kind as the resolution of $\mathscr{F}$.

\section{Computation of codimensions}

To find the codimensions of the locally closed subvarieties of $\mathrm{M}_{\mathbb{P}^{2}}(r, \chi)$ occuring in the previous sections we need to find the dimensions of the stabilizers of generic points from $W_{o}$. For actions of reductive groups it is known that a stable point has zero-dimensional isotropy group. This fact will not remain true in our context.

We begin with a lemma which seems to be known, yet we couldn't find a reference. Let $V$ be a vector space over $k$ and let $W$ be the space of $m \times n$-matrices with entries in $V$. We consider the action by conjugation on $W$ of the reductive group $G=\mathrm{GL}(m) \times \mathrm{GL}(n) / k^{*}$.

Lemma 8.1. The isotropy subgroup of a stable point from $W$ is trivial.

Proof. Let $w \in W$ be a stable matrix. Concretely, what this means, is that no matrix in the same orbit as $w$ can have a zero $p \times q$-submatrix with $\frac{p}{m}+\frac{q}{n} \geq 1$. We consider an element in the isotropy group of $w$ represented by $(g, h)$.

As $G$ is reductive, $\operatorname{Stab}_{G}(w)$ is finite, so there are $t \in k^{*}$ and an integer $r \geq 1$ such that $g^{r}=t I_{m}$ and $h^{r}=t I_{n}$. From this we see that $g$ and $h$ are diagonalizable matrices. Replacing possibly $w$ by another point in its orbit, we may assume that $g$ and $h$ are diagonal matrices. We write

$$
g=\operatorname{diag}\left(t_{1}, \ldots, t_{m}\right), \quad h=\operatorname{diag}\left(s_{1}, \ldots, s_{n}\right) .
$$

From $w=g w h^{-1}$ we see that $w_{i j}=0$ if $t_{i} \neq s_{j}$. Thus, if $t_{1}, \ldots, t_{m}, s_{1}, \ldots s_{n}$ are not all equal, then $w$ is a block matrix, say

$$
\left[\begin{array}{ll}
\star & 0 \\
0 & \star
\end{array}\right]
$$

This contradicts the stability of $w$. In conclusion, $g=t I_{m}, h=t I_{n}$; thus $(g, h)$ represents the identity of $G$.

Claim 8.2. The isotropy group of a generic semistable morphism

$$
20(-2) \oplus(n-1) \mathcal{O}(-1) \stackrel{\varphi}{\rightarrow} \mathcal{O}(-1) \oplus n \mathcal{O}, \quad \varphi_{12}=0,
$$

has dimension $n-1$. The semistability conditions are understood as in Claim 4.3. Proof. We choose a morphism $\varphi$ for which at least one of the maximal minors of $\varphi_{22}$ is nonzero. Let $(g, h)$ be in $\operatorname{Stab}_{G}(\varphi)$. Keeping the notations from Section 3 we write

$$
g^{-1}=\left[\begin{array}{cc}
g_{1} & 0 \\
u & g_{2}
\end{array}\right], \quad h=\left[\begin{array}{cc}
h_{1} & 0 \\
v & h_{2}
\end{array}\right] .
$$


We have $\varphi=h \varphi g^{-1}$ so $\varphi_{11}=h_{1} \varphi_{11} g_{1}$ and $\varphi_{22}=h_{2} \varphi_{22} g_{2}$. But $\varphi_{11}$ and $\varphi_{22}$ are stable matrices with entries in $V^{*}$. From Lemma 8.1 we get $h_{1}=t_{1}, g_{1}=t_{1}^{-1}$, $h_{2}=t_{2} I_{n}, g_{2}=t_{2}^{-1} I_{n-1}$. We have $\varphi_{21}=v \varphi_{11} t_{1}^{-1}+t_{2} \varphi_{21} t_{1}^{-1}+t_{2} \varphi_{22} u$. If $t_{1} \neq t_{2}$, then $\varphi$ is equivalent to a matrix $\varphi^{\prime}$ for which $\varphi_{21}^{\prime}=0$. This would contradict the semistability of $\varphi$. Thus $t_{1}=t_{2}=t$ and $\varphi_{22} u=-t^{-2} v \varphi_{11}$.

Recall that $\varphi_{11}=\left[X_{1}, X_{2}\right]$ with linearly independent $X_{1}, X_{2}$ in $V^{*}$. We put $\psi=\left[-X_{2}, X_{1}\right]^{T}$. From $\varphi_{22} u \psi=-t^{-2} v \varphi_{11} \psi=0$ we get $u \psi=0$ because one of the maximal minors of $\varphi_{22}$ is nonzero. Thus $u=\alpha \varphi_{11}$ with $\alpha \in \mathbf{M}_{n-1,1}(k)$. From $\left(t^{-1} v+t \varphi_{22} \alpha\right) \varphi_{11}=0$ we get $v=-t^{2} \varphi_{22} \alpha$. Thus $(g, h)$ is parametrized by $t$ and by the entries of $\alpha$, giving the claim.

The above proof worked because for $\varphi_{11}$ there existed a matrix $\psi$ such that for any $1 \times 2$-matrix $u$ with entries in $V^{*}$

$$
u \psi=0 \quad \text { implies that } u \text { is a multiple of } \varphi_{11} .
$$

For morphisms from Claim 4.7 we can take

$$
\varphi_{11}=\left[\begin{array}{lll}
X & Y & Z
\end{array}\right], \quad \psi=\left[\begin{array}{rrr}
-Y & -Z & 0 \\
X & 0 & -Y \\
0 & X & Z
\end{array}\right]
$$

and we see that (8.3) is true for $1 \times 3$-matrices $u$ with entries in $V^{*}$. We arrive at:

Claim 8.4. The isotropy group of a generic semistable morphism

$$
3 \mathcal{O}(-2) \oplus(n-2) \mathcal{O}(-1) \stackrel{\varphi}{\rightarrow} \mathcal{O}(-1) \oplus n \mathcal{O}, \quad \varphi_{12}=0,
$$

has dimension $n-2$. The semistability conditions are understood as in Claim 4.7.

For morphisms from Claims 4.8 and 4.9 the $2 \times 3$-matrix $\varphi_{11}=\left(f_{i j}\right)_{i=1,2, j=1,2,3}$ with entries in $V^{*}$ is stable. Concretely, stability here means that the maximal minors of $\varphi_{11}$ are linearly independent in $S^{2} V^{*}$. We put $f=\left[f_{1}, f_{2}, f_{3}\right]^{T}$, where

$$
f_{1}=\left|\begin{array}{ll}
f_{12} & f_{13} \\
f_{22} & f_{23}
\end{array}\right|, \quad f_{2}=\left|\begin{array}{ll}
f_{13} & f_{11} \\
f_{23} & f_{21}
\end{array}\right|, \quad f_{3}=\left|\begin{array}{ll}
f_{11} & f_{12} \\
f_{21} & f_{22}
\end{array}\right| .
$$

Clearly $\varphi_{11} f=0$. Our intention is to show that, for generic $\varphi_{11}$, and for a $1 \times 3$ matrix $u$ with entries in $V^{*}$, the equality $u f=0$ implies that $u$ is a linear combination of the rows of $\varphi_{11}$. Indeed, the condition $u f=0$ is the same as saying that the determinant of

$$
\psi=\left[\begin{array}{lll}
f_{11} & f_{12} & f_{13} \\
f_{21} & f_{22} & f_{23} \\
u_{1} & u_{2} & u_{3}
\end{array}\right]
$$

is zero. We need to prove that, modulo operations on rows and columns, $\psi$ is equivalent to a matrix having a zero row. For this we will use Remark 5.6, namely 
we will exclude the other possibilities listed there. First we see that, as the columns of $\varphi_{11}$ are linearly independent, $\psi$ cannot be equivalent to a matrix having a zero column. Nor is $\psi$ equivalent to a matrix of the form

$$
\left[\begin{array}{lll}
0 & 0 & \star \\
0 & 0 & \star \\
\star & \star & \star
\end{array}\right],
$$

for if $g \psi h$ has the above form, then all $2 \times 2$-minors positioned on the first two columns of $\psi h$ are zero. But the matrix obtained by deleting the third row of $\psi h$ is equivalent to $\varphi_{11}$, so it is stable, so its first maximal minor from the left is nonzero (in fact all its maximal minors are nonzero).

If we choose $\varphi_{11}$ generic enough, then $\psi$ is equivalent to neither $\psi_{1}$ nor $\psi_{2}$ from Remark 5.6. For instance, if $\varphi_{11}$, regarded as a map from $V^{*} \oplus V^{*} \oplus V^{*}$ to $V^{*} \oplus V^{*}$, is injective, then $\psi$ is not equivalent to $\psi_{1}$. To rule out $\psi_{2}$, we need only observe that the condition $\operatorname{det}\left(\psi_{2}\right)=0$ defines a thin subset inside the affine space with coordinates $a_{1}, \ldots a_{5}$ (notations as in Remark 5.6). In conclusion, $\psi$ is equivalent to a matrix having a zero row.

With the notations from the proof of Claim 8.2, we have $u=\alpha \varphi_{11}$ with $\alpha \epsilon$ $\mathrm{M}_{n-1,2}(k)$, and $v=-t^{2} \varphi_{22} \alpha$. We arrive at the following:

Claim 8.5. The isotropy group of a generic semistable morphism

$$
30(-2) \oplus(n-1) \mathcal{O}(-1) \stackrel{\varphi}{\rightarrow} 20(-1) \oplus n \mathbb{O}, \quad \varphi_{12}=0,
$$

has dimension $2 n-2$.

Finally, we turn to morphisms from Claim 6.9 and Claim 6.10.

Claim 8.6. The isotropy group of a generic semistable morphism

$$
(n-2) \mathcal{O}(-2) \oplus 3 \mathcal{O}(-1) \stackrel{\varphi}{\rightarrow}(n-3) \mathcal{O}(-1) \oplus 3 \mathcal{O}, \quad \varphi_{12}=0, \quad \varphi_{22}=\psi_{1},
$$

has dimension $4 n-11$.

Proof. As in Claim 8.2 we have $t_{1}=t_{2}=t$ and $v \varphi_{11} t^{-1}=-t \varphi_{22} u$. We put $\psi=[Z,-X, Y]$. From $\psi v \varphi_{11}=-t^{2} \psi \varphi_{22} u=0$ we get $\psi v=0$, because $\varphi_{11}$ can be chosen generic enough that one of its maximal minors be nonzero. From $\psi v=0$ we get $v=\varphi_{22} \alpha$ with $\alpha \in \mathrm{M}_{3, n-3}(k)$. From $\varphi_{22}\left(\alpha \varphi_{11} t^{-1}+t u\right)=0$ we get $\alpha \varphi_{11} t^{-1}+t u=[-Y, X, Z]^{T} \beta$. with $\beta \in \mathrm{M}_{1, n-2}(k)$. Thus $\operatorname{Stab}_{G}(\varphi)$ is parametrized by t, the entries of $\alpha$ and the entries of $\beta$.

Once we know the dimensions of the isotropy groups of generic points $\varphi \in W_{o}$ we can apply the obvious formula

$$
\operatorname{dim}(X)=\operatorname{dim}\left(W_{o}\right)-\operatorname{dim}(G)+\operatorname{dim}\left(\operatorname{Stab}_{G}(\varphi)\right) .
$$


We do not carry out here these computations; we refer, insted, to the table from the introduction where we have recorded the results.

\section{Duality results}

In [Freiermuth 2000] one can find a birational map of fine moduli spaces

$$
\mathbf{M}_{\mathbb{P}^{2}}(r, \chi) \rightarrow \mathbf{M}_{\mathbb{P}^{2}}(r, r-\chi)
$$

given by sending a point represented by $\mathscr{F}$ to the point represented by the dual sheaf $\mathscr{F}^{D}$. By modifying slightly the argument from [Freiermuth 2000] we will construct such birational maps also for those coarse moduli spaces occuring in Section 4. At Theorem 9.6 we will also obtain isomorphisms between dual locally closed subspaces of $\mathrm{M}_{\mathbb{P}^{2}}(r, \chi)$ and $\mathrm{M}_{\mathbb{P}^{2}}(r, r-\chi)$.

In the sequel $\mathscr{F}$ will be a coherent sheaf on $\mathbb{P}^{2}$ with pure one-dimensional support and without zero-dimensional torsion. We define its $d u a l \mathscr{F}^{D}$ by

$$
\mathscr{F}^{D}=\mathscr{E} x t_{\mathscr{O}_{\mathbb{P} 2}^{2}}^{1}\left(\mathscr{F}, \Omega_{\mathbb{P}^{2}}^{2}\right)(1) .
$$

Clearly $\mathscr{F}^{D}$ has one-dimensional support, so it has linear Hilbert polynomial. This can be computed using the following isomorphisms provided by Serre duality:

$$
\begin{array}{ll}
H^{0}\left(\mathscr{F}^{D}(-1)\right) \simeq H^{1}\left(\mathscr{F}^{*},\right. & H^{1}\left(\mathscr{F}^{D}(-1)\right) \simeq H^{0}\left(\mathscr{F}^{*},\right. \\
H^{0}\left(\mathscr{F}^{D}\right) \simeq H^{1}\left(\mathscr{F}^{*}(-1)\right)^{*}, & H^{1}\left(\mathscr{F}^{D}\right) \simeq H^{0}(\mathscr{F}(-1))^{*} .
\end{array}
$$

Thus, if $P_{\mathscr{F}}(t)=r t+\chi$, then $P_{\mathscr{F} D}(t)=r t+r-\chi$. In particular, the slopes of $\mathscr{F}$ and $\mathscr{F}^{D}$ are related by $p\left(\mathscr{F}^{D}\right)=1-p(\mathscr{F})$.

Lemma 9.1. If $\mathscr{F}$ is Cohen-Macaulay, in particular if $\mathscr{F}$ is semistable, then $\mathscr{F}^{D D} \simeq$ $\mathscr{F}$ and $\mathscr{E} x t^{2}\left(\mathscr{F}, \Omega^{2}\right)=0$.

Proof. We will apply proposition 1.1.10 from [Huybrechts and Lehn 1997]. All we need to show is that $\mathscr{F}_{F}$ satisfies the Serre condition $\mathrm{S}_{2,1}$ :

$$
\operatorname{depth}\left(\mathscr{F}_{x}\right) \geq \min \left\{2, \operatorname{dim} \mathbb{O}_{\mathbb{P}^{2}, x}-1\right\} \quad \text { for all } \quad x \in \operatorname{Supp}(\mathscr{F})
$$

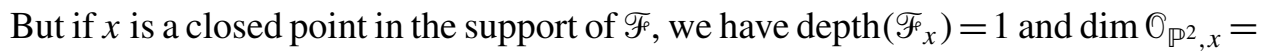
2. If $x$ is a generic point of an irreducible component of $\operatorname{Supp}(\mathscr{F})$, we have $\operatorname{dim} 0_{\mathbb{P}^{2}, x}=1$ and the above inequality is trivially fulfilled. Finally, we notice that, by virtue of Proposition 2.3, semistable sheaves are Cohen-Macaulay.

Lemma 9.2. $\mathscr{F}_{F}$ is semistable (stable) if and only if $\mathscr{F}^{D}$ is semistable (stable). 
Proof. Assume that $\mathscr{F}$ is semistable. Let $\mathscr{G}=\mathscr{F}^{D} / \mathscr{K}_{\mathcal{K}}$ be a quotient sheaf of $\mathscr{F}^{D}$. As $\mathscr{K}$ is a torsion sheaf, we have $\mathscr{H o m}\left(\mathscr{K}, \Omega^{2}\right)=0$. Applying the long exact sequence in $\mathscr{E} x t$-sheaves to the short exact sequence

$$
0 \rightarrow \mathscr{K} \rightarrow \mathscr{F}^{D} \rightarrow \mathscr{G} \rightarrow 0
$$

we see that $\mathscr{G}^{D}$ is a subsheaf of $\mathscr{F}^{D D} \simeq \mathscr{F}$. Thus

$$
1-p(\mathscr{G})=p\left(\mathscr{G}^{D}\right) \leq p(\mathscr{F})=1-p\left(\mathscr{F}^{D}\right), \quad \text { so } \quad p\left(\mathscr{F}^{D}\right) \leq p(\mathscr{G}) .
$$

This proves the semistability of $\mathscr{F}^{D}$.

Assume that $\mathscr{F}$ is not semistable. Then $\mathscr{F}$ has a quotient sheaf $\mathscr{G}$ with $p(\mathscr{G})<$ $p(\mathscr{F})$. As before, $\mathscr{G}^{D}$ is a destabilizing subsheaf of $\mathscr{F}^{D}$.

Lemma 9.3. If $\mathscr{F}_{F}$ and $\mathscr{G}$ are semistable and stable equivalent, so are $\mathscr{F}^{D}$ and $\varphi^{D}$. Proof. Consider a Jordan-Hölder filtration for $\mathscr{F}$ :

$$
0=\mathscr{F}_{0} \subset \mathscr{F}_{1} \subset \cdots \subset \mathscr{F}_{n-1} \subset \mathscr{F}_{n}=\mathscr{F} .
$$

We apply the long exact sequence in $\mathscr{E} x t$-sheaves to the exact sequences

$$
0 \rightarrow \mathscr{F}_{i} \rightarrow \mathscr{F}_{i+1} \rightarrow \mathscr{F}_{i+1} / \mathscr{F}_{i} \rightarrow 0 .
$$

As $\mathscr{F}_{i}$ is a torsion sheaf we have $\mathscr{H}_{o m}\left(\mathscr{F}_{i}, \Omega^{2}\right)=0$. As $\mathscr{F}_{i+1} / \mathscr{F}_{i}$ is semistable, we have, by Lemma $9.1, \mathscr{E} x t^{2}\left(\mathscr{F}_{i+1} / \mathscr{F}_{i}, \Omega^{2}\right)=0$. We arrive at the exact sequences

$$
0 \rightarrow\left(\mathscr{F}_{i+1} / \mathscr{F}_{i}\right)^{D} \rightarrow \mathscr{F}_{i+1}^{D} \rightarrow \mathscr{F}_{i}^{D} \rightarrow 0 .
$$

Similarly we obtain exact sequences

$$
0 \rightarrow\left(\mathscr{F} / \mathscr{F}_{i}\right)^{D} \rightarrow \mathscr{F}^{D} \rightarrow \mathscr{F}_{i}^{D} \rightarrow 0 .
$$

From these two sequences we conclude that

$$
0=\left(\mathscr{F}_{F} / \mathscr{F}_{n}\right)^{D} \subset\left(\mathscr{F}_{F} / \mathscr{F}_{n-1}\right)^{D} \subset \cdots \subset\left(\mathscr{F}_{F} / \mathscr{F}_{1}\right)^{D} \subset\left(\mathscr{F}_{F} / \mathscr{F}_{0}\right)^{D}=\mathscr{F}^{D}
$$

is a Jordan-Hölder filtration of $\mathscr{F}^{D}$ with terms $\left(\mathscr{F}_{i+1} / \mathscr{F}_{i}\right)^{D}$, the latter being stable by virtue of Lemma 9.2. The lemma follows.

Theorem 9.4. Assume that $r / 2 \leq \chi \leq r$ and that $r, \chi$ are mutually prime. Then the open dense subset of $\mathrm{M}_{\mathbb{P}^{2}}(r, \chi)$ given by the conditions

$$
h^{0}(\mathscr{F}(-1))=0, \quad h^{1}(\mathscr{F})=0, \quad h^{1}\left(\mathscr{F} \otimes \Omega^{1}(1)\right)=0,
$$

is isomorphic to the open dense subset of $\mathrm{M}_{\mathbb{P}^{2}}(r, r-\chi)$ given by the conditions

$$
h^{1}(\mathscr{F})=0, \quad h^{0}(\mathscr{F}(-1))=0, \quad h^{0}\left(\mathscr{F} \otimes \Omega^{1}(1)\right)=0 .
$$

The isomorphism is given by $[\mathscr{F}] \rightarrow\left[\mathscr{F}^{D}\right]$. 
Proof. From Lemmas 9.1, 9.2 and 9.3 we see that the map $\delta$ given by $[\mathscr{F}] \rightarrow\left[\mathscr{F}^{D}\right]$ is well defined and a bijection between the two open sets from the theorem, which we call $\mathrm{M}_{o}(r, \chi)$ and $\mathrm{M}_{o}(r, r-\chi)$.

Every sheaf $\mathscr{F}$ from $\mathrm{M}_{o}(r, \chi)$ has Beilinson resolution

$$
0 \rightarrow(r-\chi) \mathcal{O}(-2) \oplus(2 \chi-r) \mathcal{O}(-1) \stackrel{\varphi}{\rightarrow} \chi \mathcal{O} \rightarrow \mathscr{F} \rightarrow 0 .
$$

The long exact sequence in $\mathscr{E} x t$-sheaves gives the resolution

$$
0 \rightarrow \chi \mathscr{O}(-2) \stackrel{\varphi^{D}}{\rightarrow}(r-\chi) \mathcal{O} \oplus(2 \chi-r) \mathcal{O}(-1) \rightarrow \mathscr{F}^{D} \rightarrow 0,
$$

where $\varphi^{D}$, viewed as a matrix, is simply the transpose of $\varphi$. The set of morphisms $\varphi$ occuring above forms an open subset $W_{o}$ inside the vector space of morphisms

$$
(r-\chi) \mathcal{O}(-2) \oplus(2 \chi-r) \mathcal{O}(-1) \rightarrow \chi \mathscr{O} .
$$

On $W_{o} \times \mathbb{P}^{2}$ we consider the coherent sheaf $\widetilde{\mathscr{F}}$ given by the exact sequence

$$
\mathcal{O}_{W_{o}} \otimes(r-\chi) \mathcal{O}_{\mathbb{P}^{2}}(-2) \oplus \mathcal{O}_{W_{o}} \otimes(2 \chi-r) \mathcal{O}_{\mathbb{P}^{2}}(-1) \stackrel{\Phi}{\rightarrow} \chi \mathcal{O}_{W_{o} \times \mathbb{P}^{2}} \rightarrow \stackrel{\widetilde{F}_{F}}{\rightarrow} 0 .
$$

On each fiber $\{\varphi\} \times \mathbb{P}^{2}$ the restriction of $\Phi$ is $\varphi$. Similarly we construct the dual family as the cokernel

$$
\mathcal{O}_{W_{o}} \otimes \chi \mathcal{O}_{\mathbb{P}^{2}}(-2) \stackrel{\Phi^{D}}{\rightarrow} \mathcal{O}_{W_{o}} \otimes(r-\chi) \mathbb{O}_{\mathbb{P}^{2}} \oplus \mathcal{O}_{W_{o}} \otimes(2 \chi-r) \mathcal{O}_{\mathbb{P}^{2}}(-1) \rightarrow \widetilde{\mathscr{F}} D \rightarrow 0
$$

of a morphism $\Phi^{D}$ which restricts to $\varphi^{D}$ on each fiber $\{\varphi\} \times \mathbb{P}^{2}$. Clearly $\widetilde{\mathscr{F}}$ and $\widetilde{\mathscr{F}}^{D}$ are $W_{o}$-flat, so they induce morphisms

$$
\rho: W_{o} \rightarrow \mathbf{M}_{o}(r, \chi), \quad \rho^{D}: W_{o} \rightarrow \mathbf{M}_{o}(r, r-\chi) .
$$

We have $\delta \circ \rho=\rho^{D}$.

Next we recall from Section 2 that $\mathrm{M}_{\mathbb{P}^{2}}(r, \chi)$ is the good quotient of an open subset $R$ inside a certain quotient scheme. Let $S$ be the preimage of $\mathrm{M}_{o}(r, \chi)$ under the quotient map $R \rightarrow \mathrm{M}_{\mathbb{P}^{2}}(r, \chi)$. The map

$$
\pi: S \rightarrow \mathrm{M}_{o}(r, \chi)
$$

ia a good quotient map. Let $U$ be the restriction to $S \times \mathbb{P}^{2}$ of the universal quotient family on $R \times \mathbb{P}^{2}$. From the fact that all restrictions of $u$ to the fibers $\{s\} \times \mathbb{P}^{2}$, $s \in S$, have Beilinson resolution (9.5) we deduce, as in the proof of Proposition 7.14 , the existence of locally defined morphisms $\varsigma: S_{o} \rightarrow W_{o}$ satisfying

$$
\rho \circ \zeta=\pi
$$


The morphisms $\rho^{D} \circ \varsigma$ glue to a globally defined morphism $\pi^{D}$ making the diagram

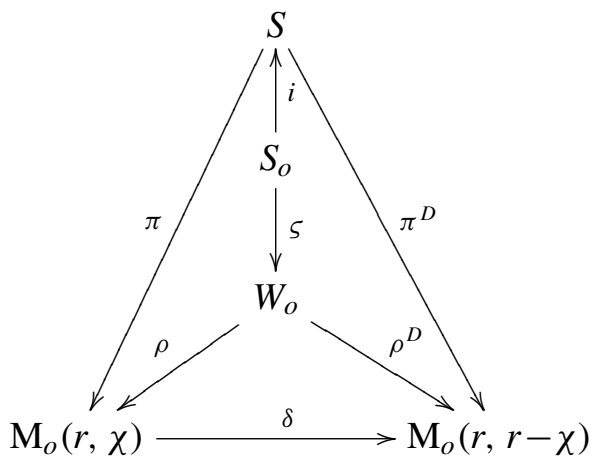

commute. Thus $\delta$ is the map induced by $\pi^{D}$ via the universal property of the quotient map $\pi$. As such, $\delta$ must be a morphism. By symmetry, its inverse must be a morphism, too.

The above theorem first appeared in [Freiermuth 2000]. Its proof given there is simpler and makes use of the universal families on the fine moduli spaces. Our argument, though more cumbersome, has the following advantage: it works also in the case when $r, \chi$ are not mutually prime, as long as we know that all sheaves giving a point in $\mathrm{M}_{o}$ have the same kind of Beilinson complex.

Theorem 9.6. Let $X$ be the locally closed subvariety of $\mathrm{M}_{\mathbb{P}^{2}}(r, \chi)$ given by the conditions

$$
h^{0}(\mathscr{F}(-1))=a, \quad h^{0}(\mathscr{F})=b, \quad h^{0}\left(\mathscr{F} \otimes \Omega^{1}(1)\right)=c .
$$

Assume that every sheaf giving a point in $X$ satisfies the above conditions. This is the case, for instance, when $r, \chi$ are mutually prime. Then $X$ is isomorphic to the locally closed subvariety $X^{D}$ of $\mathrm{M}_{\mathbb{P} 2}(r, r-\chi)$ given by the conditions

$$
h^{1}(\mathscr{F})=a, \quad h^{1}(\mathscr{F}(-1))=b, \quad h^{1}\left(\mathscr{F} \otimes \Omega^{1}(1)\right)=c .
$$

The isomorphism is given by $[\mathscr{F}] \rightarrow\left[\mathscr{F}^{D}\right]$. Here $X$ and $X^{D}$ are equipped with their canonical reduced structures.

Proof. Assume that $X$ is nonempty. We repeat the arguments from Theorem 9.4.

We consider vector bundles $\mathscr{E}^{i}$ on $\mathbb{P}^{2}, i=-2,-1,0,1$, which are decomposable as direct sums of line bundles. We assume that for each $\mathscr{F}$ giving a point in $X$ there is a complex

$$
0 \rightarrow \mathscr{E}^{-2} \stackrel{\varphi}{\rightarrow} \mathscr{E}^{-1} \stackrel{\varphi^{\prime}}{\rightarrow} \mathscr{E}^{0} \stackrel{\varphi^{\prime \prime}}{\rightarrow} \mathscr{E}^{1} \rightarrow 0
$$


which is exact, except at $\mathscr{E}^{0}$, where the cohomology is $\mathscr{F}$. For instance, we could choose $\mathscr{C}^{i}$ to be the bundles $\mathscr{C}^{i}$ occuring in the Beilinson complex (4.1). Let $W_{o}$ be the set of the above complexes.

$W_{o}$ will play the same role as in the proof of Theorem 9.4. The existence of $\rho: W_{o} \rightarrow X$ is clear by construction. To finish the proof, we only need to construct $\rho^{D}$ satisfying $\delta \circ \rho=\rho^{D}$. For this purpose we will show that $H_{o m}\left({ }_{-}, \Omega^{2}\right)(1)$ applied to (9.7) gives a complex

$$
0 \rightarrow \mathscr{E}_{D}^{1} \rightarrow \mathscr{E}_{D}^{0} \rightarrow \mathscr{E}_{D}^{-1} \rightarrow \mathscr{E}_{D}^{-2} \rightarrow 0
$$

that is exact, except at $\mathscr{E}_{D}^{-1}$, where the cohomology is $\mathscr{F}^{D}$.

We consider the long exact sequences in $\mathscr{E} x t\left(-, \Omega^{2}\right)$-sheaves induced by the short exact sequences

$$
\begin{gathered}
0 \rightarrow \mathscr{E}^{-2} \rightarrow \mathscr{E}^{-1} \rightarrow \mathscr{A} \rightarrow 0, \\
0 \rightarrow \mathscr{B} \rightarrow \mathscr{E}^{0} \rightarrow \mathscr{E}^{1} \rightarrow 0, \\
0 \rightarrow \mathscr{A} \rightarrow \mathscr{B} \rightarrow \mathscr{F} \rightarrow 0 .
\end{gathered}
$$

Since $\mathscr{E} x t^{j}\left(\mathcal{O}(d),{ }_{-}\right)=0$ for $j \geq 1$, we have $\mathscr{E} x t^{j}\left(\mathscr{C}^{i}, \Omega^{2}\right)=0$ for $j \geq 1$. The second sequence gives $\mathscr{E} x t^{1}\left(\mathscr{B}, \Omega^{2}\right)=0$. In view of Lemma 9.1, the semistability of $\mathscr{F}$ leads to $\mathscr{E} x t^{2}\left(\mathscr{F}, \Omega^{2}\right)=0$. The third sequence gives $\mathscr{E} x t^{1}\left(\mathscr{A}, \Omega^{2}\right)=0$. Thus we arrive at the exact sequences

$$
\begin{gathered}
0 \rightarrow \operatorname{Hom}\left(\mathscr{A}, \Omega^{2}\right) \rightarrow \mathscr{H o m}\left(\mathscr{E}^{-1}, \Omega^{2}\right) \rightarrow \mathscr{H o m}\left(\mathscr{E}^{-2}, \Omega^{2}\right) \rightarrow 0, \\
0 \rightarrow \mathscr{H o m}\left(\mathscr{E}^{1}, \Omega^{2}\right) \rightarrow \mathscr{H o m}\left(\mathscr{E}^{0}, \Omega^{2}\right) \rightarrow \mathscr{H o m}\left(\mathscr{B}, \Omega^{2}\right) \rightarrow 0, \\
0 \rightarrow \operatorname{Hom}\left(\mathscr{B}, \Omega^{2}\right) \rightarrow \mathscr{H o m}\left(\mathscr{A}, \Omega^{2}\right) \rightarrow \mathscr{E} x t^{1}\left(\mathscr{F}, \Omega^{2}\right) \rightarrow 0,
\end{gathered}
$$

which immediately yield (9.8).

We mentioned at the end of Section 7 that all locally closed subvarieties $X$ occuring in Section 4, satisfy the hypotheses of the above theorem. Indeed, it can be verified in each case that the cohomological properties defining $X$ are closed under stable equivalence. As a consequence, all locally closed subvarieties $X \subset$ $\mathbf{M}_{\mathbb{P}^{2}}(r, \chi)$ occuring in Sections 4-6, with the possible exception of the subvarieties in $\mathrm{M}_{\mathbb{P} 2}(3 r, 3), r=3,4,5$, occuring in section 6, are isomorphic to their duals $X^{D}$. In particular, Theorem 9.4 remains true for the following choices of multiplicity and Euler characteristic: $(6,4),(8,6),(9,6)$. We obtain:

Corollary 9.9. The spaces $\mathrm{M}_{\mathbb{P}^{2}}(r, \chi)$ and $\mathrm{M}_{\mathbb{P}^{2}}(r, r-\chi)$ are birational for $(r, \chi)=$ $(6,4),(8,6),(9,6)$.

Here is another application of Theorem 9.6: the closed subset of $\mathrm{M}_{\mathbb{P}^{2}}(4,1)$ given by the conditions $h^{0}(\mathscr{F}(-1))=0, h^{1}(\mathscr{F})=1$ (the condition $h^{0}\left(\mathscr{F} \otimes \Omega^{1}(1)\right)=1$ is automatically fulfilled), is isomorphic to the closed subset of $\mathrm{M}_{\mathbb{P}^{2}}(4,3)$ given by 
the conditions $h^{1}(\mathscr{F})=0, h^{0}(\mathscr{F}(-1))=1$. This we proved earlier at Corollary 7.9 and Corollary 7.10 by means of their description as geometric quotients.

Let $W_{o}^{D}$ denote the set of complexes (9.8), i.e. the set of complexes obtained by applying Hom $\left(_{-}, \Omega^{2}\right)(1)$ to the complexes from $W_{o}$. If we identify $W_{o}$ with a certain subset of triples of matrices $\left(\varphi, \varphi^{\prime}, \varphi^{\prime \prime}\right)$ inside the vector space

$$
W=\operatorname{Hom}\left(\mathscr{E}^{-2}, \mathscr{E}^{-1}\right) \times \operatorname{Hom}\left(\mathscr{E}^{-1}, \mathscr{\mathscr { C }}^{0}\right) \times \operatorname{Hom}\left(\mathscr{C}^{0}, \mathscr{C}^{1}\right),
$$

then $W_{o}^{D}$ is just the subset of triples of transposed matrices $\left(\varphi^{\prime \prime}{ }^{T}, \varphi^{\prime T}, \varphi^{T}\right)$ inside the vector space

$$
W^{D}=\operatorname{Hom}\left(\mathscr{E}_{D}^{1}, \mathscr{E}_{D}^{0}\right) \times \operatorname{Hom}\left(\mathscr{E}_{D}^{0}, \mathscr{E}_{D}^{-1}\right) \times \operatorname{Hom}\left(\mathscr{E}_{D}^{-1}, \mathscr{E}_{D}^{-2}\right) .
$$

Thus transposition gives an isomorphism of $W_{o}$ with $W_{o}^{D}$, both equipped with their canonical reduced structures induced by the ambient spaces $W$ and $W^{D}$.

On $W_{o}$ and on $W_{o}^{D}$ we have the canonical action of the (usually nonreductive) algebraic group

$$
G=\operatorname{Aut}\left(\mathscr{E}^{-2}\right) \times \operatorname{Aut}\left(\mathscr{E}^{-1}\right) \times \operatorname{Aut}\left(\mathscr{E}^{0}\right) \times \operatorname{Aut}\left(\mathscr{E}^{1}\right) .
$$

From the proofs of Theorem 9.4 and Theorem 9.6 we extract the following:

Proposition 9.10. Let $X$ be as in Theorem 9.6. Assume that a good quotient of $W_{o}$ by $G$ exists and is isomorphic to $X$. Then a good quotient of $W_{o}^{D}$ by $G$ exists and is isomorphic to $X^{D}$.

For every subset $X \subset \mathrm{M}_{\mathbb{P}^{2}}(r, \chi)$ described in section 7 as a good (geometric) quotient, we have a dual description of $X^{D} \subset \mathrm{M}_{\mathbb{P}^{2}}(r, r-\chi)$ as a good (geometric) quotient. For better understanding let us introduce to a polarization $\Lambda$ of type $(2,1)$ or type $(2,2)$ its dual polarization $\Lambda^{D}$ of type $(1,2)$, respectively type $(2,2)$ :

$$
\text { for } \Lambda=\left(\lambda_{1}, \lambda_{2}, \mu_{1}\right) \text { we put } \Lambda^{D}=\left(\lambda_{1}^{D}, \mu_{1}^{D}, \mu_{2}^{D}\right)=\left(\mu_{1}, \lambda_{2}, \lambda_{1}\right) \text {; }
$$

for $\Lambda=\left(\lambda_{1}, \lambda_{2}, \mu_{1}, \mu_{2}\right)$ we put $\Lambda^{D}=\left(\lambda_{1}^{D}, \lambda_{2}^{D}, \mu_{1}^{D}, \mu_{2}^{D}\right)=\left(\mu_{1}, \mu_{2}, \lambda_{2}, \lambda_{1}\right)$.

If $W_{o}$ is defined by semistability conditions expressed in terms of $\Lambda$, then $W_{o}^{D}$ is defined by semistability conditions expressed in terms of $\Lambda^{D}$. We list below the consequences of Proposition 9.10 for the cases of generic sheaves:

Corollary 9.11. The open dense subset of $\mathrm{M}_{\mathbb{P}^{2}}(n+1,1), n \geq 2$, given by the condition $h^{1}(\mathscr{F})=0$, is isomorphic to $W_{o} / G$, where $W_{o}$ is the set of injective morphisms

$n \mathscr{O}(-2) \stackrel{\varphi}{\rightarrow}(n-1) \mathcal{O}(-1) \oplus \mathcal{O}, \quad \varphi \in W^{\mathrm{ss}}(G, \Lambda), \Lambda=\left(\lambda_{1}, \mu_{1}, \mu_{2}\right), 0<\mu_{2}<\frac{1}{n}$.

The open dense subset of $\mathrm{M}_{\mathbb{P}^{2}}(n+2,2), n=3,4,5,6$, given by the conditions

$$
h^{0}(\mathscr{F}(-1))=0, \quad h^{1}(\mathscr{F})=0, \quad h^{0}\left(\mathscr{F} \otimes \Omega^{1}(1)\right)=0,
$$


is isomorphic to $W_{o} / / G$, where $W_{o}$ is the set of injective morphisms $n \mathcal{O}(-2) \stackrel{\varphi}{\rightarrow}(n-2) \mathcal{O}(-1) \oplus 2 \mathcal{O}, \quad \varphi \in W^{\mathrm{ss}}(G, \Lambda), \Lambda=\left(\lambda_{1}, \mu_{1}, \mu_{2}\right), \frac{1}{2 n}<\mu_{2}<\frac{1}{n}$.

The open dense subset of $\mathrm{M}_{\mathbb{P}^{2}}(n+3,3), n=4,5$, given by the conditions

$$
h^{0}(\mathscr{F}(-1))=0, \quad h^{1}(\mathscr{F})=0, \quad h^{0}\left(\mathscr{F} \otimes \Omega^{1}(1)\right)=0,
$$

is isomorphic to $W_{o} / G$, where $W_{o}$ is the set of injective morphisms $n \mathcal{O}(-2) \stackrel{\varphi}{\rightarrow}(n-3) \mathcal{O}(-1) \oplus 3 \mathcal{O}, \quad \varphi \in W^{\mathrm{ss}}(G, \Lambda), \Lambda=\left(\lambda_{1}, \mu_{1}, \mu_{2}\right), \frac{2}{3 n}<\mu_{2}<\frac{1}{n}$.

One final example of a quotient we were not able to obtain in Section 6: the subset of $\mathrm{M}_{\mathbb{P}^{2}}(6,3)$ given by the conditions $h^{0}(\mathscr{F}(-1))=1, h^{1}(\mathscr{F})=0$ (the condition $h^{1}\left(\mathscr{F} \otimes \Omega^{1}(1)\right)=3$ is automatically fulfilled), is isomorphic to $W_{o} / G$, where $W_{o}$ is the set of injective morphisms

$$
4 \mathbb{O}(-2) \stackrel{\varphi}{\rightarrow} 3 \mathcal{O}(-1) \oplus \mathbb{O}(1), \quad \varphi \in W^{\mathrm{ss}}(G, \Lambda), \quad \Lambda=\left(\lambda_{1}, \mu_{1}, \mu_{2}\right), \quad 0<\mu_{2}<\frac{1}{4} .
$$

Applying Proposition 9.10 to the quotients from Section 6 we get descriptions for the subsets in $\mathrm{M}_{\mathbb{P}^{2}}(n+3, n), n=4,5,7,8,10,11,13,14$, given by the conditions $h^{0}(\mathscr{F}(-1))=0, h^{1}(\mathscr{F})=1$. We omit the details.

\section{Acknowledgements}

The author thanks J.-M. Drézet for many useful comments. The referee pointed out several improvements, including a simplification of the proof of Claim 4.3, for which the author is grateful.

\section{References}

[Barth 1977] W. Barth, "Moduli of vector bundles on the projective plane", Invent. Math. 42 (1977), 63-91. MR 57 \#324 Zbl 0386.14005

[Chang 1983] M.-C. Chang, "Stable rank 2 bundles on $\mathbf{P}^{3}$ with $c_{1}=0, c_{2}=4$, and $\alpha=1$ ", Math. Z. 184:3 (1983), 407-415. MR 85g:14022 Zbl 0507.14006

[Drézet 1987] J.-M. Drézet, "Fibrés exceptionnels et variétés de modules de faisceaux semi-stables sur $\mathbf{P}_{2}$ (C)", J. Reine Angew. Math. 380 (1987), 14-58. MR 89e:14016 Zbl 0613.14013

[Drézet 1991] J.-M. Drézet, "Variétés de modules extrémales de faisceaux semi-stables sur $\mathbf{P}_{2}(\mathbf{C})$ ", Math. Ann. 290:4 (1991), 727-770. MR 92m:14017 Zbl 0755.14005

[Drézet 1998] J.-M. Drézet, "Quotients algébriques par des groupes non réductifs et variétés de modules de complexes”, Internat. J. Math. 9:7 (1998), 769-819. MR 99m:14026 Zbl 0947.14009

[Drézet 2000] J.-M. Drézet, "Espaces abstraits de morphismes et mutations", J. Reine Angew. Math. 518 (2000), 41-93. MR 2001c:14023 Zbl 0937.14030

[Drézet and Trautmann 2003] J.-M. Drézet and G. Trautmann, "Moduli spaces of decomposable morphisms of sheaves and quotients by non-reductive groups", Ann. Inst. Fourier (Grenoble) 53:1 (2003), 107-192. MR 2004c:14092 Zbl 1034.14023 
[Freiermuth 2000] H.-G. Freiermuth, On the moduli space $M_{P}\left(\mathbb{P}_{3}\right)$ of semi-stable sheaves on $\mathbb{P}_{3}$ with Hilbert polynomial $P(m)=3 m+1$, Diplomarbeit, Univ. Kaiserslautern, 2000.

[Freiermuth and Trautmann 2004] H.-G. Freiermuth and G. Trautmann, "On the moduli scheme of stable sheaves supported on cubic space curves”, Amer. J. Math. 126:2 (2004), 363-393. MR 2005b:14026 Zbl 1069.14012

[Hulek 1979] K. Hulek, "Stable rank-2 vector bundles on $\mathbf{P}_{2}$ with $c_{1}$ odd", Math. Ann. 242:3 (1979), 241-266. MR 80m:14011 Zbl 0407.32013

[Huybrechts and Lehn 1997] D. Huybrechts and M. Lehn, The geometry of moduli spaces of sheaves, Aspects of Mathematics E31, Vieweg, Braunschweig, 1997. MR 98g:14012 Zbl 0872.14002

[Le Potier 1993] J. Le Potier, "Faisceaux semi-stables de dimension 1 sur le plan projectif", Rev. Roumaine Math. Pures Appl. 38:7-8 (1993), 635-678. MR 95a:14014 Zbl 0815.14029

[Maican 2000] M. Maican, Variation of GIT-quotients. examples, techniques and applications to moduli spaces, Diplomarbeit, Univ. Kaiserslautern, 2000.

[Newstead 1978] P. E. Newstead, Introduction to moduli problems and orbit spaces, Tata Lectures on Mathematics and Physics 51, Narosa, New Delhi, 1978. MR 81k:14002 Zbl 0411.14003

[Okonek et al. 1980] C. Okonek, M. Schneider, and H. Spindler, Vector bundles on complex projective spaces, Progress in Mathematics 3, Birkhäuser, Boston, 1980. MR 81b:14001 Zbl 0438.32016

[Simpson 1994a] C. T. Simpson, "Moduli of representations of the fundamental group of a smooth projective variety, I", Inst. Hautes Études Sci. Publ. Math. 79 (1994), 47-129. MR 96e:14012 Zbl 0891.14005

[Simpson 1994b] C. T. Simpson, "Moduli of representations of the fundamental group of a smooth projective variety, II", Inst. Hautes Études Sci. Publ. Math. 80 (1994), 5-79. MR 96e:14013 Zbl 0891.14006

Received May 14, 2007. Revised October 19, 2007.

MARIO MAICAN

202 SuRgE BUILDING

UNIVERSITY OF CALIFORNIA

RIVERSIDE, CA 92521

UNITED STATES

mario.maican@ucr.edu 